\title{
MICROANALYSES OF \\ Voting, Regulation and Higher Education
}

\section{Dissertation}

zur Erlangung des wirtschaftswissenschaftlichen Doktorgrades der Wirtschaftswissenschaftlichen Fakultät der Universität Göttingen

vorgelegt von

Johannes Meya

aus Heide

Göttingen, 2015 
Erstgutachter: Prof. Dr. Robert Schwager

Zweitgutachter: Prof. Panu Poutvaara, Ph.D.

Tag der mündlichen Prüfung: 1. Juni 2015 


\section{Acknowledgements}

I want to thank my first supervisor Robert Schwager for his support and advice during the last years. Furthermore, I am grateful to Panu Poutvaara for being my second supervisor and to Olaf Korn for being the third member of my examination committee.

I want to thank my co-authors Kamila Danilowicz-Gösele, Katharina Lerche, Panu Poutvaara and Robert Schwager for the great collaboration. Moreover, I am grateful to the whole team of the Chair of Public Economics for manifold support, discussions, the outstanding working environment and a great time.

Finally, I gratefully acknowledge financial support for the work presented in Chapters 2, 3 and 4 from the German Federal Ministry of Education and Research under grant number 01PW11004. 


\section{Contents}

1 Introduction $\quad 1$

2 Pocketbook Voting and Social Preferences in Referenda 6

2.1 Introduction . . . . . . . . . . . . . . 6

2.2 Data . . . . . . . . . . . . . . . . . 11

2.2.1 Institutional background and data collection . . . . . . . . 11

2.2 .2 Dataset I . . . . . . . . . . . . . . . . 13

2.2 .3 Dataset II . . . . . . . . . . . . . . 16

2.3 The big picture . . . . . . . . . . . . . . . . 18

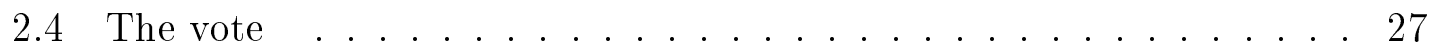

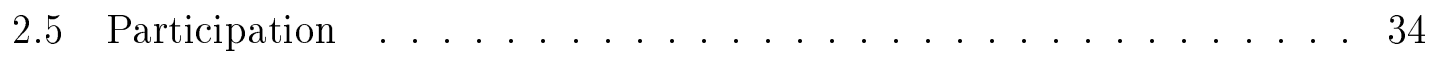

2.6 Conclusion . . . . . . . . . . . . . . . 37

Appendix to Chapter 2 . . . . . . . . . . . . . . 39

3 Determinants of Students' Success at University 51

3.1 Introduction . . . . . . . . . . . . . . 51

3.2 Literature . . . . . . . . . . . . . . . . . 53

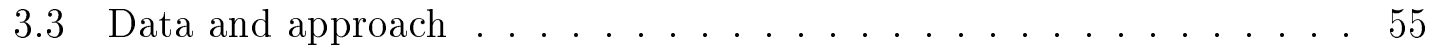

3.3.1 Variable description and institutional background . . . . . 56

3.3 .2 Summary statistics . . . . . . . . . . . . 58

3.3.3 Empirical setup . . . . . . . . . . . . . 60

3.4 Results . . . . . . . . . . . . . . . . 61

3.4 .1 University level . . . . . . . . . . . . . . 61

3.4 .2 Faculties . . . . . . . . . . . . . . 63

3.5 Discussion and policy implications . . . . . . . . . . . . 72 
Appendix to Chapter $3 \ldots \ldots \ldots \ldots \ldots$. . . . . . . . . . . . . 75

4 The Second Dividend of Studying Abroad:

The Impact of International Student Mobility on Academic Per$\begin{array}{lr}\text { formance } & \mathbf{8 0}\end{array}$

4.1 Introduction . . . . . . . . . . . . . . . . . 80

4.2 Data description . . . . . . . . . . . . . . . . 82

4.3 Empirical framework . . . . . . . . . . . . . . 87

4.4 Results . . . . . . . . . . . . . . . . . . 92

4.5 Sensitivity to unobserved heterogeneity . . . . . . . . . . . . 99

4.6 Conclusion . . . . . . . . . . . . . . . . . . . . . . 101

Appendix to Chapter $4 \ldots \ldots . \ldots . \ldots . \ldots 103$

5 Dynamics of Yardstick Regulation:

Historical Cost Data and the Ratchet Effect 106

5.1 Introduction . . . . . . . . . . . . . . . 106

5.2 Description of the model . . . . . . . . . . . . . . . 109

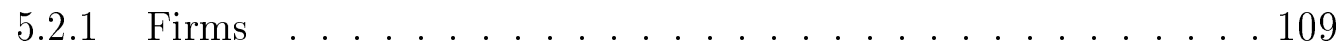

5.2 .2 Regulatory rules . . . . . . . . . . . . . 112

5.3 Equilibrium analysis . . . . . . . . . . . . . . 113

5.3 .1 Optimal slack . . . . . . . . . . . . . . . 115

5.3.2 Steady state equilibria . . . . . . . . . . . . . 124

5.3 .3 Comparative dynamics . . . . . . . . . . . . . 128

5.4 Conclusion . . . . . . . . . . . . . . . . . . . . 129

Appendix to Chapter 5 . . . . . . . . . . . . . . 130

$\begin{array}{ll}\text { References } & 143\end{array}$ 


\section{List of Tables}

2.1 Summary statistics - Dataset I . . . . . . . . . . . 15

2.2 Summary statistics - Dataset II . . . . . . . . . . . . . 17

2.3 Pocketbook voting - Dataset I . . . . . . . . . . . . 20

2.4 Voting in favor but unwilling to buy: importance of social preferences 21

2.5 Voting in favor but unwilling to buy: different social preferences . . 23

2.6 Pocketbook voting - Dataset II . . . . . . . . . . . 25

2.7 Social preferences and protest among winners and losers - Dataset II 26

2.8 Train ticket - Dataset I . . . . . . . . . . . . . . 29

2.9 Bus ticket - Dataset I . . . . . . . . . . . . . . . 29

2.10 Culture ticket - Dataset I . . . . . . . . . . . . . 30

2.11 Bahn ticket - Dataset II . . . . . . . . . . . . . . . 32

2.12 Taking part - Dataset II . . . . . . . . . . . . . . 34

2.13 Taking part, reduced sample - Dataset II . . . . . . . . . 36

2.A.1 Train ticket - Dataset I, coefficients for Table 2.8 . . . . . . . . . 42

2.A.2 Bus ticket - Dataset I, coefficients for Table 2.9 . . . . . . . . 43

2.A.3 Culture ticket - Dataset I, coefficients for Table 2.10 . . . . . . . 44

2.A.4 Bahn ticket - Dataset II, coefficients for Table 2.11 . . . . . . . 45

2.A.5 Taking part - Dataset II, coefficients for Table $2.12 \ldots . . . .45$

2.A.6 Taking part, reduced sample - Dataset II, coefficients for Table 2.1346

2.A.7 General political preferences - Dataset I, coefficients . . . . . . 47

2.A.8 Fields of study - Dataset I, coefficients . . . . . . . . . . . 48

2.A.9 Political preferences and fields of study - Dataset I, coefficients . . 49

2.A.10 General political preferences - Dataset II, coefficients . . . . . . . 50

$3.1 \quad$ Summary statistics . . . . . . . . . . . . . . . 59 
3.2 University level . . . . . . . . . . . . . . . . . . 62

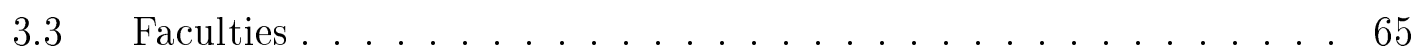

3.4 Graduation by faculties . . . . . . . . . . . . 66

3.5 Predicted probabilities of graduation by faculties . . . . . . . 68

3.6 Grades by faculties . . . . . . . . . . . . . . . . 69

3.A.1 University level - Coefficients for Table $3.2 \ldots . . . . .77$

3.A.2 Faculties - Coefficients for Table $3.3 \ldots \ldots$. . . . . . 78

3.A.3 Graduation by faculties - Coefficients for Table 3.4 . . . . . . . 79

4.1 Summary statistics . . . . . . . . . . . . 86

4.2 Final university grade - OLS results . . . . . . . . . . . . . . . . . . 92

4.3 Probability of studying abroad . . . . . . . . . . . . . 94

4.4 Final university grade - matching results . . . . . . . . . . . . 95

4.5 Transferring grades . . . . . . . . . . . . . . 96

4.6 Graduating in time - probit results . . . . . . . . . . . . 98

4.7 Graduating in time - matching results . . . . . . . . . . . . 99

4.8 Sensitivity analysis . . . . . . . . . . . . . . 100

4.A.1 Balancing table for nearest neighbor matching . . . . . . . . . 103

4.A.2 Determinants of transferring grades . . . . . . . . . . . 105 


\section{List of Figures}

$2.1 \quad \mathrm{Map} \ldots \ldots \ldots \ldots \ldots \ldots \ldots$

2.2 Intensity of use and yes votes - Dataset I . . . . . . . . . . . 19

2.3 Rationalizing votes - Dataset I . . . . . . . . . . . . 21

2.4 Savings and share of yes votes - Dataset II . . . . . . . . . . . 24

2.5 Turnout and savings - Dataset II . . . . . . . . . . . . 27

3.1 Grades at selected faculties . . . . . . . . . . . . . . 70

4.A.1 Distribution of the propensity score before and after matching . . . 104 


\section{List of Abbreviations}

FOC First order condition

GPA Grade point average

OLS Ordinary least squares 


\section{Chapter 1}

\section{Introduction}

This book presents economic microanalyses of voting, regulation and higher education. Arguably, considerable parts of society might not necessarily approach these issues from an economic perspective. However, self-evidently, all of them play major roles in modern societies and involve substantial resources. Consequently, they are affected by economic considerations and, in turn, shape the economy. More fundamentally, the concept of this book is to examine these topics of relevance to society with economic approaches and techniques. It is about individual decisions, institutions, and the incentives the latter create.

Institutions provide the framework in which individuals, economic agents, make their choices. Most of these institutions, formal as well as informal, are essentially exogenous from the perspective of the individual. At the same time, they are to a large extent endogenous to the will of society. For instance, in referenda voters directly shape institutions and public policy, and hence decide on how things are organized. Taking this as a starting point, we begin our analyses with an investigation of the drivers of individual voting decisions in referenda. Afterwards, we take the institutional setting as given and analyze the determinants of students' academic performance at university. Subsequently, we examine a specific aspect of tertiary education: temporary study-related visits abroad. Here we focus on the consequences of implicit incentives and individual choices on academic performance measures in a given institutional framework. Finally, we examine the incentive structure in a specific regulatory setting in which firms are rewarded based on their individual 
performance relative to the performance of other firms.

Although there are differences in the specific focus, the following chapters all address questions of public economics. While investigating public decision-making is certainly in the core of public economics, the economic analysis of higher education is shared between two neighboring fields; labor and public economics. Similarly, Laffont (1994, p. 507) describes regulation as "the public economics face of industrial organization." This, in turn, renders regulation the industrial organization face of public economics. The analysis of government, public policy and institutions are at the heart of this book.

The unifying methodological feature of our analyses is the microperspective taken. Society as a whole is often interested in, so to say, 'aggregate' results; for instance the outcome of a referendum or the share of students graduating. However, similarly to the sum of the smaller pieces that make a mosaic, it is the sum of individual voting decisions that pass or fail a proposal, and individual students graduating. The microperspective taken provides a deeper understanding of institutions, incentive structures and individual behavior. On a more aggregate level, our results, therefore, allow to derive predictions in a larger context, to come to policy implications, and to evaluate institutions.

Chapters 2-4 are based on empirical results, whereas Chapter 5 is a purely theoretical analysis employing dynamic game theory. To keep each chapter comprehensive on its own, we review the relevant literature separately in the respective chapters. In the following paragraphs, we provide a summary of the remaining parts of this book and our main results.

As pointed out above, in democratic societies, many, if not most, formal institutions are themselves subject to the will of the people and may be subject to change. In representative democracies, the electorate delegates the right to pass and change laws to some group of people; for instance members of a parliament. Referenda, in contrast, give a decision itself into the hands of the electorate. In Chapter 2, we shed light on the motives that drive individual voting behavior in direct democratic decisions. We investigate the role of monetary self-interest and social preferences in four referenda held among university students. In these referenda, the student body decides about collective purchases of flat rate tickets for local trains, local buses or 
a culture ticket that offers free or highly discounted entrance to cultural institutions like theaters. If passed, buying the respective ticket becomes compulsory for all students of the university, including those who do not use it or voted against it. Hence, these referenda share essential features of decisions on tax-financed publicly provided goods - yet, voters should be much better informed about their personal costs and benefits in our setting.

Chapter 2 is based on two surveys with more than a thousand respondents each. One survey was conducted as an exit poll, the other survey was done online so as to include non-voters, too, and thereby to allow for the analysis of the turnout decision. For each referendum, individual usage data provides a measure of personal monetary benefits the ticket in question provides a voter with. The main findings of this chapter can be summarized as follows: We find that students who gain a lot from having a ticket take part in a referendum with higher probability, suggesting instrumental voting. Our investigation of the individual votes cast provides strong evidence for pocketbook voting, i.e. voting in favor if one gains personally and against if one loses from having the respective ticket. At the same time, social preferences and motives play an important role, too, and shift the vote of a sizable minority of voters against their narrowly defined self-interest. Moreover, our analysis suggests that social preferences were likely or came very close to being pivotal in half of the referenda studied.

Chapter 3 presents a microanalysis of the determinants of academic success of university students. It is based on a unique administrative dataset collected at a German university containing detailed, anonymous information on more than 12,000 student careers. In line with the literature, that is reviewed in detail in Chapter 3 , we find that the grade of the high school leaving degree is strongly related to students' success in higher education. This holds true for both kinds of measures employed, the probability to graduate and the final university grade. The impact of socio-economic variables is, in contrast, relatively small.

Looking separately at the different fields of study, substantial differences emerge, suggesting differences in the teaching and examination cultures: At some faculties, most students can expect to graduate with strongly differentiated grades, for instance, economic sciences or forest sciences. At others, like social sciences or hu- 
manities, chances to reach the degree are lower. However, if graduating, grades are relatively good conditional on high school performance. At a third group of faculties, for instance, mathematics and physics, graduation seems very challenging for weaker students and they can hardly expect good grades.

Building on the results presented in Chapter 3, Chapter 4 focuses on the impact of a temporary study-related visit at a university in a foreign country on students' academic performance. In this part of the book, we base our analysis on more than 2,500 students who successfully completed their bachelor studies as for them some common rules apply, for instance, a regular study period of six semesters. The main empirical challenge arising in this context is that students who go abroad for parts of their studies are not a random selection. Due to the rich dataset at hand, we can apply a propensity score matching strategy to account for this self-selection.

The key finding in this chapter is that a sojourn has a positive impact on the final university grade. We call this effect the second dividend of studying abroad in addition to personal experience and development. Our analysis suggests that this effect results from selective transferring of grades which are relatively good compared to the average performance of the individual student. However, this second dividend seems to come at a cost: we find that a sojourn has a negative impact on the probability of graduating within the standard time period.

Finally, in Chapter 5, we turn towards an area where incentives provided by institutions are more explicit. This part of the book is about incentive regulation for natural monopolies. Such a regulation is explicitly designed to address and to provide incentives for economic agents in order to reach a specific goal. In this part of the book, we do not rely on empirical results. Instead, we present a purely theoretical analysis based on techniques from game theory and dynamic programming.

Regulation of natural monopolies is characterized by an asymmetric information structure: On the one side, there is a regulator who tries to reach, for instance, economic efficiency or a pricing that is considered fair. On the other side, there are one or more firms that have superior knowledge about their technology and own interests that potentially deviate from those of the regulator. In this chapter, we analyze the incentive structure for firms under a yardstick regulation that uses historical cost data. The idea of this kind of regulation, pioneered by Shleifer (1985), is to have 
several local natural monopolies 'compete' via a regulation in which constraints for each firm, for instance prices allowed to be charged, are defined based on cost data of other comparable firms. In static settings, yardstick regulation decouples the performance of a firm from its constraints and thereby provides strong incentives for efficient production.

The main result in this chapter is that the incentive structure is different from this in a dynamic setting where historical cost data is used to define constraints. We show that firms can influence their own future constraints by affecting other firms' constraints and behavior. Therefore, a ratchet effect, that should be overcome by this regulation, can occur. As a consequence, inefficient equilibria can exist without any form of collusion or threat. Comparing two variants of yardstick regulation, we show that this problem is more severe if the best of all other firms instead of the average of the other firms is used as the yardstick. ${ }^{1}$

\footnotetext{
${ }^{1}$ The summaries of Chapters 2-5 heavily borrow from the abstracts of the discussion paper versions of these chapters Meya et al. (2015), Danilowicz-Gösele et al. (2014), Meya and Suntheim (2014) and Meya (2015) respectively.
} 


\section{Chapter 2}

\section{Pocketbook Voting and Social Preferences in Referenda*}

\subsection{Introduction}

Referenda are an integral part of democracy in several jurisdictions, such as Switzerland and California. For example, by bounding the property tax rate, Proposition 13 in 1978 decisively shaped local public finances in California (see California State Board of Equalization, 2012, p. 1). On the expenditure side, major examples include referenda on the Gotthard train tunnel in Switzerland in 1992 or on the high-speed railway in California (Proposition 1A) in 2008. In other countries, referenda have usually been restricted to constitutional issues such as membership in the European Union. In recent years, however, an increasing number of countries have also held referenda on non-constitutional issues, in particular public infrastructure projects. For instance, local referenda on transportation took place in 2005 (Edinburgh road tolls referendum) and in 2008 (Greater Manchester transport referendum) in the United Kingdom, Italy voted on nuclear power and water service tariffs in 2011, and a German state held a referendum on a major railway project (Stuttgart 21) in 2011.

*This chapter originates from joint work with Panu Poutvaara and Robert Schwager (see Meya et al., 2015). 
Despite their increasing popularity, referenda, and in particular those on fiscal issues, are controversial. Proponents welcome the broad public debate and the democratic legitimacy of decisions ensured by referenda. In this spirit, Rousseau (2012 [1762], p. 65) argued already 250 years ago: "Every law the people has not ratified in person is null and void - is, in fact, not a law." Opponents fear that uninformed or ideologically biased citizens either do not bother to turn out or end up making inefficient or inequitable decisions. Schumpeter (1994 [1942], p. 261) was convinced that "[the private citizen] expends less disciplined effort on mastering a political problem than he expends on a game of bridge." We contribute to this debate by empirically analyzing the motives for participating in a referendum and for voting against or in favor of a proposal. Our results show that 'pocketbook voting', that is voting along monetary interests, is predominant. However, social considerations such as the benefits and costs of other voters or the promotion of some common good are also present, and sometimes even pivotal.

We consider referenda on flat rate tickets for students at Goettingen University in Germany. If passed, such a ticket gives all students the right to unlimited use of some facility such as public transportation or cultural amenities. The price of the ticket is very attractive compared to individual purchases, but buying the ticket becomes compulsory for every student once the majority voted in favor. Such tickets therefore share essential features of tax-financed public projects like the examples mentioned above. By collectively procuring the ticket, per capita cost of the respective facility are reduced so that frequent users stand to gain substantially from an approval by the majority. At the same time, some voters will use the facility in question very little or not at all, but are still forced to pay as much for it as anyone else.

Investigating these referenda is particularly promising since they refer to easyto-understand public policy decisions. In particular, in our setting, voters knew exactly what a ticket would cost and benefits were clearly defined. Opposed to that, if the vote took place, say, on a big infrastructure project, costs and benefits would be uncertain. Different voting decisions could also then reflect different subjective expectations on possible deviations from projections and differences in risk attitudes. In this sense, the referenda we study are like a 'laboratory' for direct democratic decisions, where confounding influences are reduced to a minimum. 
Our main dataset covers votes on tickets for regional trains, cultural facilities and local buses, taken in 2013. The second dataset is on a referendum about a train ticket, held in 2010. Our analysis is based on two surveys conducted after the votes. Whereas the survey in 2013 was a paper-based exit poll, the dataset from 2010 was collected online. Therefore, this second dataset also encompasses students who did not participate in the referendum, allowing for the analysis of the turnout decision.

Both datasets contain detailed information about usage behavior, votes, political preferences and other characteristics of more than a thousand respondents each. In the main dataset, usage is reported in categorical variables. In the second dataset, we construct a detailed monetary measure of individual benefits conferred by the ticket. To do so, we combine information on the number of trips to parents with regular ticket prices that we derive from parental address data. Additionally, students were asked to what extent the interests of others shaped their voting decision. Further motives include social or political goals, such as promoting local cultural life and expressing protest against the pricing policy of the rail company.

Our primary focus is on the probability of voting in favor of a ticket. We find strong evidence for pocketbook voting. Most students voted in line with monetary interests. In the main dataset, a student who uses a facility very often is between 52 and 76 percentage points more likely to vote in favor of the corresponding ticket than one who never uses it. In the second dataset, a 10 percent increase in cost savings conferred by the train ticket raises the probability of voting in favor by 0.7 to 0.8 percentage points. This translates into widely differing predictions, given that savings vary between zero and more than three thousand euros per year.

However, our results show that monetary self-interest is not the whole story. While party preferences are mostly not relevant for the voting decision, variables capturing social preferences, such as altruism and merit good considerations, show highly significant and economically relevant effects. A sizeable share of students who do not gain in monetary terms from a ticket voted in favor because of such motives. The analysis suggests that social preferences were likely pivotal in one out of four referenda and were close to being pivotal in another one. Pocketbook voting and social preferences together can rationalize almost all votes cast.

In the second part of this chapter, the dependent variable is participation in 
the referendum. We find that the probability of taking part increases in individual stakes, measured by the absolute value of the difference between the benefits conferred by the ticket and its price. This suggests some degree of instrumental turnout. Furthermore, our analysis shows that students with very large positive benefits drive this result. Additionally, there is evidence that some students did turn out in order to protest against the train company's pricing policy.

This chapter contributes to the literature on the motives of voters. A central question in this literature is to what extent citizens vote according to their narrowly defined self-interest and to what extent voting decisions are driven by social considerations.

Pocketbook voting is the starting point in models of income redistribution building on Meltzer and Richard (1981), in the theory of probabilistic voting (Lindbeck and Weibull, 1993; Dixit and Londregan, 1996) and in median voter models of local public finance (Romer et al., 1992; Epple and Romano, 1996; Epple et al., 2001). Several authors specifically aim at empirically detecting this motive in referenda. In an early contribution, Deacon and Shapiro (1975) find that voters in cities connected to the BART transport system in the San Francisco area were more likely to favor a proposition which would shift petrol tax revenues to public transportation. Similarly, voters in precincts which are located close to sports stadiums are more positive towards subsidizing them (Coates and Humphreys, 2006). Intriguingly, according to Potrafke (2013), this does not hold for concert halls. Vlachos (2004) concludes that voting patterns in the Swedish referendum on the EU membership are in line with conflicting regional interests. Similar to these authors, we find evidence for pocketbook voting, but we go further by analyzing individual voting and turnout decisions rather than relying on regional vote shares.

Even more importantly, we study the role of social preferences, which has so far been neglected in the analysis of real world referenda. This contrasts with experimental studies on voting behavior which typically find that in addition to monetary self-interest, voting reflects various kinds of social preferences. In particular, Tyran and Sausgruber (2006) show that inequity aversion in the sense of Fehr and Schmidt (1999) is important in laboratory elections. Introducing a novel random price voting mechanism, Messer et al. (2010) conclude that subjects' behavior is better explained 
by pure altruism than by inequity aversion. Balafoutas et al. (2013) find that while voting on redistribution is mostly predicted by self-interest, there is greater support for redistribution when inequalities are arbitrary than when they reflect performance in an experimental task. This is in line with what Fong (2001) and Alesina and Angeletos (2005) derive from survey evidence.

Literature on economic voting has mostly focused on representative democracy. Lewis-Beck and Stegmaier (2007) survey more than 400 studies without finding much evidence for pocketbook voting. Most of the studies in their analysis suffer from severe identification problems: they assume that voters attribute all changes in their financial situation to the policies of the incumbent government. When analyzing well-defined past policies, Levitt and Snyder (1997), Richter (2006), Manacorda et al. (2011), Kriner and Reeves (2012), Pop-Eleches and Pop-Eleches (2012), De La O (2013) and Zucco (2013) find that voters increase their support to the government if they have benefited from its transfers in the past. Thachil (2014) shows that poor people vote for elite parties because organizations linked to these parties provide them local public services. Elinder et al. (2015) find strong evidence for prospective pocketbook voting: voters already react when reforms appear as electoral promises.

In general, it should be noted that pocketbook voting does not exclude taking into account also what is good for others. Fiorina (1978) and Markus (1988) conclude that both self-interest and conviction on what is good for the society matter for American voters. In Sweden, most survey respondents admitted that own interest mattered either as much as conviction or more when they chose the party to vote for (Carlsson and Johansson-Stenman, 2010).

We also contribute to the literature on voter turnout. Already Downs (1957) and Riker and Ordeshook (1968) presented the idea that the more is at stake, the more likely an individual should vote. Indeed, Andersen et al. (2014) observe that turnout in Norwegian local elections is higher in jurisdictions with high hydropower income. Alternative explanations suggest that voting is driven by ethical concerns (Harsanyi, 1980; Coate and Conlin, 2004), social pressure (Gerber et al., 2008; Funk, 2010), expressive motivations (Brennan and Buchanan, 1984; Brennan and Hamlin, 1998; Coate et al., 2008; Hillman, 2010), or social identity (Ben-Bassat and Dahan, 2012; Hillman et al., forthcoming). We also find that the likelihood of participating 
increases in the personal stake. However, there is also evidence that some voters turned out to protest against the train company's pricing policy. This behavior can be tactical in order to improve students' bargaining position. Alternatively, it can be motivated by expressive concerns.

A number of studies have found that voting decisions react to national economic conditions. Seminal contributions to this literature include Key (1966), Barro (1973), Kinder and Kiewiet (1979), Fiorina (1981), and Rogoff and Sibert (1988). Since good economic conditions generally also benefit the individual voter, such sociotropic voting may reflect self-interest or altruism. Recently, Margalit (2013) and Ansell (2014) have shown that those personally affected by macroeconomic developments adjust their attitude towards redistributive policies. While our setting is unrelated to macroeconomic performance, our analysis complements this line of work by distinguishing between pocketbook voting and various social preferences.

Finally, this chapter is also broadly related to the literature which investigates the impact of direct democracy on the public budget. Referenda are associated with lower public spending both in the US (Matsusaka, 1995) and in Switzerland (Feld and Kirchgässner, 2001; Feld and Matsusaka, 2003; Funk and Gathmann, 2011). The focus of this chapter differs from these contributions in that we do not aim at comparing direct and representative democracy. Nevertheless, our finding that those who benefit most are most likely to vote suggests that direct democracy does not necessarily result in underspending.

The remainder of this chapter is structured as follows. Section 2.2 presents the data and some background information. In Section 2.3, we give a descriptive overview of voting motives. Econometric results on the voting and participation decisions are presented in Sections 2.4 and 2.5 respectively. Section 2.6 concludes.

\section{$2.2 \quad$ Data}

\subsubsection{Institutional background and data collection}

Goettingen University periodically holds referenda among students on whether they should collectively purchase flat rate tickets, called 'Semestertickets'. Such a ticket 
gives all students of the university the right to use some specific service as often as they wish. The price of the ticket is very attractive compared to prices for individual use, but once a ticket is accepted in the referendum, its price is collected as part of the registration fee from all students with only very few exemptions.

Referenda are usually held yearly during at least three consecutive days in January at several locations on campus, and postal vote is possible. For accepting a ticket, a double threshold must be passed: at least $50 \%$ of votes must be in favor of the ticket and, at the same time, at least $15 \%$ of students must vote in favor.

We analyze votes on tickets for regional trains, local buses, and cultural amenities. The main dataset, which we label Dataset I in the following, is based on a survey related to referenda on all three tickets which took place in January 2013. The bus ticket would be valid on all buses within Goettingen and two nearby villages. The culture ticket offers free or highly discounted entrance to a number of cultural institutions and events such as theaters, museums and concerts. The train ticket, which is described in more detail below, grants free travel on local trains. The train ticket had been in place without interruption since 2004, the cultural ticket was introduced in October 2012, while the bus ticket would have been a novelty.

The prices per semester amounted to 8.55 euros for the cultural ticket, 25.80 euros for the bus ticket and 95.04 euros for the train ticket. About $36 \%$ of almost 25,600 students took part in each referendum, and two out of three referenda were close. While the culture ticket just passed with $53 \%$ approval, the bus ticket failed with $46 \%$ support. An overwhelming majority of $82 \%$ voted in favor of the train ticket.

Dataset I was collected using exit polls. After leaving the polling place, students were approached by members of the survey team and asked to take part in a paper based survey. To preserve anonymity, cubicles similar to polling booths were installed. Participation was incentivized by a lottery with prizes of 200, 100 and 50 euros.

Dataset II was collected after a referendum held in May 2010 on a train ticket only. The ticket cost 42.24 euros per semester and differed in scope from the one voted on in 2013, as explained below. Of about 22,800 students registered at that time, $24 \%$ participated in the referendum, thereof $82 \%$, voting yes.

In order to obtain data on the voting and traveling behavior of students an 
anonymous online survey was conducted. Different to exit polls, this way also nonvoters could be included in the dataset. To incentivize participation, students were invited to take part in a lottery of 250 euros and 15 times 2 tickets for a local cinema. The survey was open from July 6 till November 11, 2010.

The tracks and stations covered by the train tickets are depicted in Figure 2.1. Before 2010, there had been one train ticket covering, with only minor changes, all tracks depicted, served by several operators. The vote in 2010, however, was preceded by complaints from students' representatives about the price which Deutsche Bahn charged for its part of the tracks. As a result, the ticket was split in two. The first one covered the offer by two companies named Metronom and Cantus, henceforth called MetroCan ticket (tracks drawn as solid red lines in Figure 2.1), and was approved in January 2010. The second one covered the tracks served by Deutsche Bahn and two smaller companies (drawn as dashed lines in blue in Figure 2.1). The latter companies are jointly referred to as Bahn throughout this chapter. After some negotiations with Bahn, a referendum on the Bahn ticket was held in May 2010. Information in Dataset II refers to this referendum. In later years, including 2013, the ticket proposal again covered all tracks.

\subsubsection{Dataset I}

After dropping students who did not provide any voting decision, Dataset I contains 1334 observations. Summary statistics are shown in Table 2.1. Within our sample, the shares of yes votes for all three tickets are slightly higher than the respective overall shares. Students in the dataset would have just passed the bus ticket which narrowly missed the $50 \%$ approval threshold in the referendum. However, as our sample contains detailed information on one seventh of all votes cast, we are confident that these deviations are of minor importance when analyzing individual voting decisions.

The intensity of use is measured by categorical variables defined differently for the tickets (see Table 2.1). For the train and culture tickets, students were asked about their use within the last 12 months and the year before the ticket had been introduced, respectively. For the bus ticket, the intensity of use refers to teaching 
Figure 2.1: Map

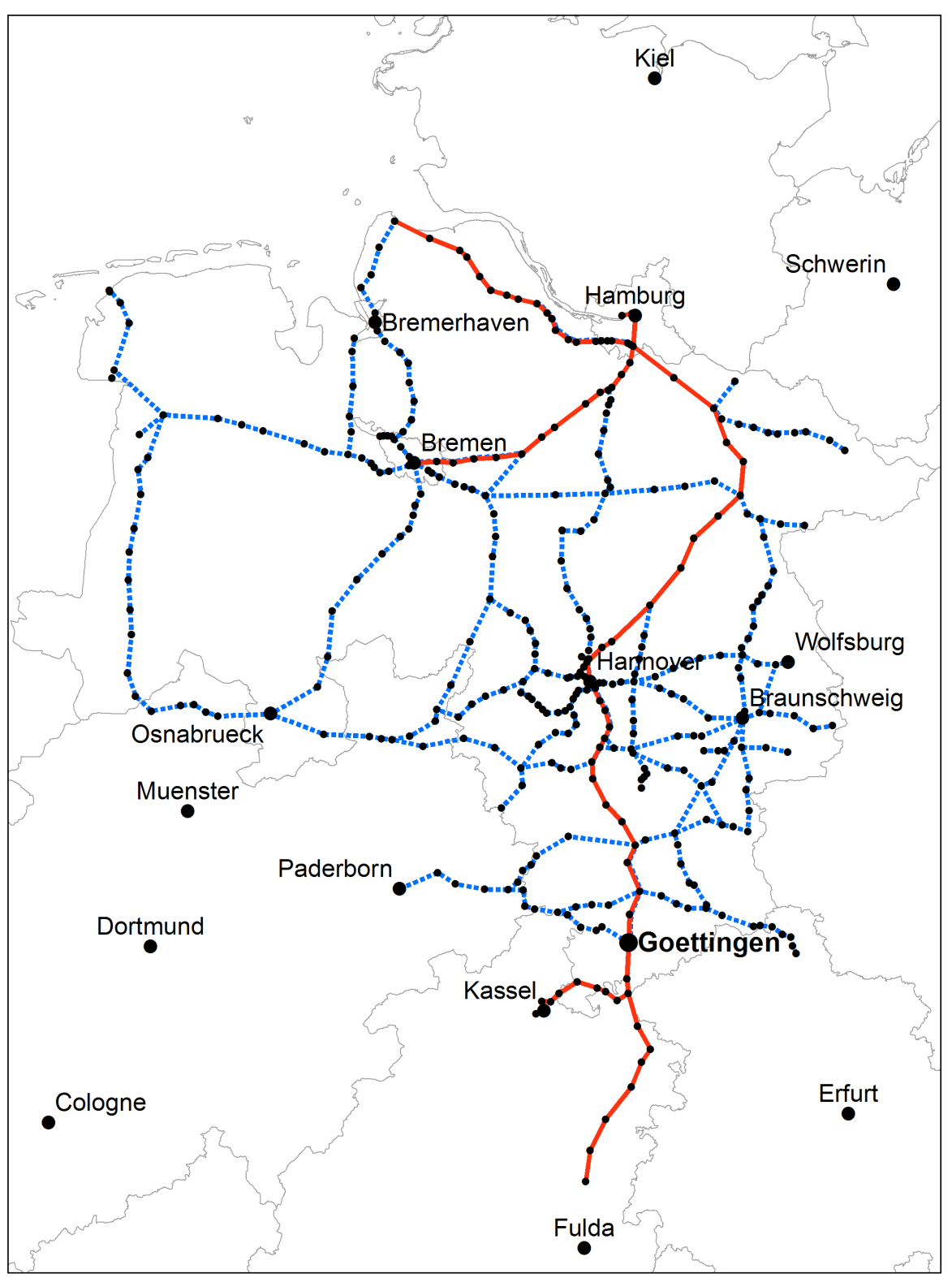

The tracks covered by the Bahn (dashed blue lines) and MetroCan (solid red lines) tickets. Grey lines are state boundaries. 
Table 2.1: Summary statistics - Dataset I

\begin{tabular}{|c|c|c|}
\hline Variable & $\mathrm{N}$ & Mean \\
\hline \multicolumn{3}{|l|}{ Train ticket } \\
\hline Train ticket: yes & 1252 & 0.86 \\
\hline Would buy it & 1248 & 0.64 \\
\hline Never & 1321 & 0.07 \\
\hline Rarely $(\leq 5 /$ year $)$ & 1321 & 0.17 \\
\hline Sometimes (monthly) & 1321 & 0.32 \\
\hline Often (weekly) & 1321 & 0.21 \\
\hline Very often ( $\geq 2 /$ week $)$ & 1321 & 0.24 \\
\hline Savings of others important & 1292 & 0.47 \\
\hline Environment important & 1284 & 0.38 \\
\hline \multicolumn{3}{|l|}{ Bus ticket $\diamond$} \\
\hline Bus ticket: yes & 1246 & 0.51 \\
\hline Would buy it & 1276 & 0.37 \\
\hline Never & 1329 & 0.40 \\
\hline Rarely ( 1 or $2 /$ semester) & 1329 & 0.24 \\
\hline Sometimes (monthly) & 1329 & 0.13 \\
\hline Often (weekly) & 1329 & 0.09 \\
\hline Very often ( $\geq 2$ /week) & 1329 & 0.14 \\
\hline Savings of others important & 1280 & 0.23 \\
\hline Strengthening bus important & 1245 & 0.12 \\
\hline \multicolumn{3}{|l|}{ Culture ticket } \\
\hline Culture ticket: yes & 1283 & 0.54 \\
\hline Would buy it & 1233 & 0.44 \\
\hline Never & 1234 & 0.56 \\
\hline Rarely ( 1 or $2 /$ year) & 1234 & 0.25 \\
\hline Sometimes ( 3 to $5 /$ year) & 1234 & 0.12 \\
\hline Often (6 to $10 /$ year) & 1234 & 0.04 \\
\hline Very often $(>10 /$ year $)$ & 1234 & 0.03 \\
\hline Savings of others important & 1235 & 0.24 \\
\hline Others should go important & 1201 & 0.29 \\
\hline Strengthening local culture important & 1229 & 0.39 \\
\hline \multicolumn{3}{|l|}{ Control variables } \\
\hline Female & 1276 & 0.50 \\
\hline Freshman & 1318 & 0.30 \\
\hline Christian Democrats & 1140 & 0.26 \\
\hline Social Democrats & 1140 & 0.29 \\
\hline Liberal Democrats & 1140 & 0.04 \\
\hline Green & 1140 & 0.31 \\
\hline Left & 1140 & 0.05 \\
\hline Other parties & 1140 & 0.05 \\
\hline Economic sciences & 1322 & 0.30 \\
\hline Social sciences & 1322 & 0.24 \\
\hline Forestry/Agriculture & 1322 & 0.06 \\
\hline Humanities & 1322 & 0.27 \\
\hline Geology/Geography & 1322 & 0.03 \\
\hline Law & 1322 & 0.11 \\
\hline Natural sciences & 1322 & 0.08 \\
\hline Other fields & 1322 & 0.03 \\
\hline
\end{tabular}

$\diamond$ Intensity of the use of the bus ticket refers to lecture period. 
periods during term. Since first-year students had not been at the university for a full year, we control for freshman status in the regression analysis.

For each of the three tickets the survey contains a question capturing whether the respondent considered savings of other students important for his or her vote. Answers to these questions were given on a four point Likert scale ranging from 'not important' to 'important'. Furthermore, we asked about other motives, like environmental aspects in the case of the train ticket or strengthening local transportation or local cultural life, using the same Likert scale. In Table 2.1, we give the shares of students who replied that the respective consideration was important. Additional control variables are gender and fields of study. ${ }^{2}$ Moreover, political preferences were captured by a question on how the respondent would vote in a federal election if this were to take place the following Sunday. Finally, students were asked if they would buy the ticket for themselves in case the ticket would be rejected in the referendum but would be available to be bought for the same price on individual basis.

\subsubsection{Dataset II}

Summary statistics for Dataset II are reported in Table 2.2. This dataset consists of 1189 observations after cleaning the data. ${ }^{3}$ Out of these, 828 students took part in the referendum. This shows an overrepresentation of voters in our sample. At the same time, it allows us to base our analysis on detailed information of almost one sixth of all voters of the referendum. Among the voters in the sample, the share of yes votes is $68 \%$, and hence smaller than the share of yes votes in the polling box.

The key variable in this dataset is the individual savings of each student. We construct an objective measure of the savings associated with the Bahn ticket by combining the number of trips to the respondents' parents using this ticket within the last 12 months with the price that would have to be paid in absence of the ticket. ${ }^{4}$ We focus on trips to parents as these are the most common trips students

\footnotetext{
${ }^{2}$ Due to the high number of polling stations the survey team could not cover all during opening hours on all three days. Therefore the faculties of law, humanities, economic sciences and social sciences are over-represented in the dataset.

${ }^{3}$ See Appendix 2.A.I for a detailed description.

${ }^{4} \mathrm{~A}$ detailed description of the calculation of savings is included as Appendix 2.A.II.
} 
Table 2.2: Summary statistics - Dataset II

\begin{tabular}{|c|c|c|c|c|}
\hline \multirow[b]{2}{*}{ Variable } & \multicolumn{2}{|c|}{ All } & \multicolumn{2}{|c|}{ Vote on Bahn ticket $=1$} \\
\hline & $\mathrm{N}$ & Mean & $\mathrm{N}$ & Mean \\
\hline Vote on Bahn ticket & 1189 & 0.70 & 828 & 1.00 \\
\hline Bahn ticket: yes & 818 & 0.68 & 818 & 0.68 \\
\hline Savings $\diamond$ & 1189 & 255.09 & 828 & 302.92 \\
\hline Stakes $\$$ & 1189 & 259.31 & 828 & 297.56 \\
\hline Own price threshold $\$$ & 1125 & 69.76 & 783 & 72.31 \\
\hline Exp. ave. price threshold ${ }^{\curvearrowright}$ & 1099 & 63.20 & 764 & 63.03 \\
\hline Leisure/work & 1189 & 0.06 & 828 & 0.06 \\
\hline Visiting others & 1174 & 0.65 & 819 & 0.67 \\
\hline Female & 1176 & 0.57 & 817 & 0.54 \\
\hline Freshman & 1099 & 0.15 & 768 & 0.18 \\
\hline Altruist $(-)$ & 1074 & 0.14 & 741 & 0.13 \\
\hline Altruist $(+)$ & 1074 & 0.34 & 741 & 0.33 \\
\hline Protest & 1189 & 0.21 & 828 & 0.24 \\
\hline Christian Democrats & 911 & 0.21 & 645 & 0.22 \\
\hline Social Democrats & 911 & 0.24 & 645 & 0.27 \\
\hline Liberal Democrats & 911 & 0.11 & 645 & 0.11 \\
\hline Green & 911 & 0.35 & 645 & 0.33 \\
\hline Left & 911 & 0.03 & 645 & 0.02 \\
\hline Other parties & 911 & 0.05 & 645 & 0.05 \\
\hline
\end{tabular}

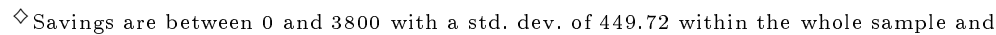
487.99 among the voters, $\$$ stakes are between 0 and 3715.52 with a std. dev. of 405.06 and 444.15 respectively, ${ }^{*}$ own price thresholds are between 0 and 750 with a std. dev. of 65.22 and 64.14 respectively, ${ }^{\circ}$ expected average price thresholds are between 10 and 720 with a std. dev. of 47.92 and 46.76 respectively.

make. Moreover, the two bigger cities close to Goettingen, namely Hannover and Kassel, which might be attractive leisure destinations, can be reached using the MetroCan ticket (see Figure 2.1). For the analysis of the decision to take part in the referendum, we transform the savings variable by subtracting the ticket price per year and taking the absolute value. Thereby, we gain a quantitative measure of the stakes a student has in the referendum.

Control variables in this dataset include gender and the party the student voted for in the last federal election in 2009. Further variables contain information on whether or not the student visited people other than his or her parents using the ticket and whether the student is a freshman. This is relevant since first-year students in the dataset started university in October 2009 only. Thus, they could not use the ticket for a full year.

The questionnaire also allowed students to enter free text on the main reasons to vote in favor or against the Bahn ticket. In order to use this qualitative information, 
a content analysis was done to identify relevant topics. Afterwards, three raters independently coded all answers with respect to whether a topic did apply. Finally, an indicator variable, that is equal to one if at least two out of the three raters independently identified the topic in the statement given and zero otherwise, was defined.

We use two variables resulting from this qualitative analysis: leisure/work captures if the student mentioned leisure activities other than visiting people, such as exploring the region or work-related aspects. The second item emerging from the content analysis is protest: some students expressed their unwillingness to accept the price of the ticket or were afraid that acceptance of the conditions would foster future price increases. Among the voters, the share of students referring to leisure/work and protest is about $6 \%$ and $24 \%$, respectively.

Dataset II includes information on the highest prices at which students would vote in favor of the Bahn ticket and their beliefs about the corresponding average of fellow students. We also asked students how they weighted these two amounts in their vote. If the decision was not only influenced by his or her own amount, a student is classified as altruist. The resulting group of altruists is then split into those who think that students on average gain from this ticket, and those who think that students on average lose. Accordingly, altruist $(+)$ is equal to one if the student based his or her decision not only on his or her own amount and believes that the price threshold of fellow students is on average greater than the price, and zero otherwise. We define altruist(-) analogously. If the student did not vote, these two indicator variables are based on the hypothetical question how he or she would have weighted these amounts.

\subsection{The big picture}

In this section, we take a closer look at the data in a descriptive analysis. The big picture that emerges is that there is strong evidence for pocketbook voting, but that social preferences also play an important role. For a first impression of the relevance of pocketbook voting, consider Figure 2.2. This figure depicts the share of yes votes in Dataset I depending on how intensively the voter used the service on which the 
vote took place. There is a strong link between own use and the likelihood of voting yes. For each ticket, more than $90 \%$ of those who used the service very often voted in favor, while the share of yes votes varies between $24 \%$ and $32 \%$ for those who never used the service.

Figure 2.2: Intensity of use and yes votes - Dataset I

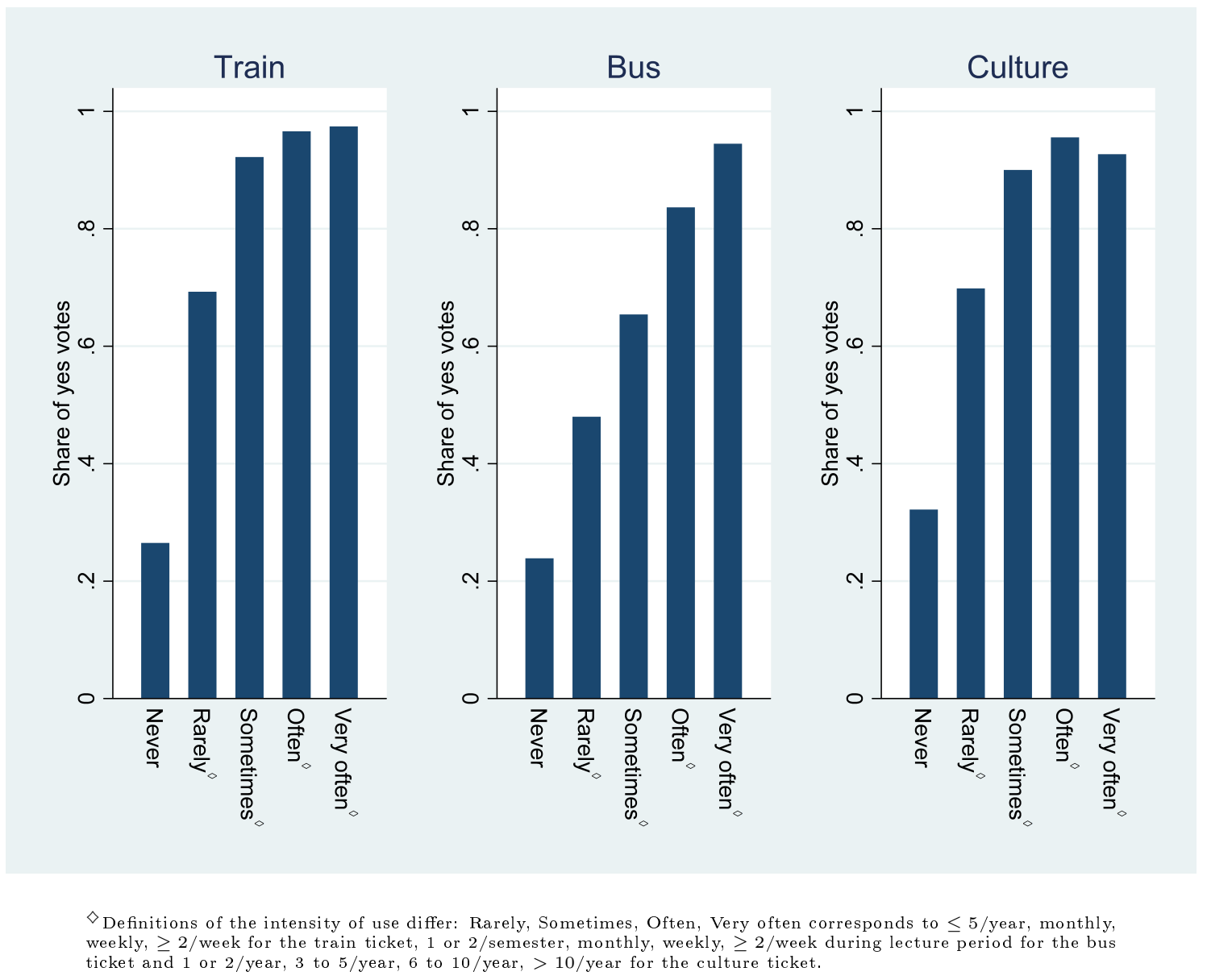

To understand to what extent pocketbook considerations can explain voting, we relate the voting decision to the binary variable stating whether the respondent would have bought the ticket individually in case it would be rejected in the referendum. If voting followed exclusively pocketbook considerations, we would expect those who vote in favor to be willing to buy the ticket also if this was available for 
Table 2.3: Pocketbook voting - Dataset I

\begin{tabular}{c|cc|c}
\hline \hline \multicolumn{4}{|c}{ Train ticket } \\
\hline \multirow{2}{*}{ Buy it } & \multicolumn{2}{|c}{ Vote } & \multirow{2}{*}{ Total } \\
& No & Yes & \\
\hline No & 158 & 256 & 414 \\
Yes & 11 & 755 & 766 \\
\hline Total & 169 & 1,011 & 1,180 \\
\hline \hline
\end{tabular}

\begin{tabular}{c|cc|c}
\hline \hline \multicolumn{4}{|c}{ Bus ticket } \\
\hline \multirow{2}{*}{ Buy it } & \multicolumn{2}{|c}{ Vote } & \multirow{2}{*}{ Total } \\
& No & Yes & \\
\hline No & 572 & 160 & 732 \\
Yes & 21 & 441 & 462 \\
\hline Total & 593 & 601 & 1,194 \\
\hline \hline
\end{tabular}

\begin{tabular}{c|cc|c}
\hline \hline \multicolumn{4}{c}{ Culture ticket } \\
\hline \multirow{2}{*}{ Buy it } & \multicolumn{2}{|c}{ Vote } & \multirow{2}{*}{ Total } \\
& No & Yes & \\
\hline No & 519 & 147 & 666 \\
Yes & 27 & 495 & 522 \\
\hline Total & 546 & 642 & 1,188 \\
\hline \hline
\end{tabular}

an individual purchase, and those who vote against to be unwilling to do so. Table 2.3 shows that $93 \%$ to $96 \%$ of those who voted against a semester ticket would also decline an opportunity to buy it privately. Remarkably, $23 \%$ to $27 \%$ of those who voted in favor of a ticket would not be willing to buy it privately for the same price. Taken together, about 30\% of respondents voted differently as a citizen compared with the choice that they would make as a private consumer. We conjecture that social preferences explain most of this difference.

To test our conjecture, we next counted which fraction of those who voted in favor of each ticket but would not buy it privately reported that at least one social motive was important (strong social preferences), and which fraction reported that none was important but at least one was somewhat important (moderate social preferences). The social preferences we consider relate to altruistic concerns towards other students when a respondent mentions that savings of others are a motivation to vote in favor of a ticket, or to a common good, when a respondent supports the service in question because it is good for the environment (in case of the train ticket) or because he or she wants to strengthen the local culture or the bus system. Social preferences also encompass responses that other students should use cultural services more often.

Table 2.4 shows that strong social preferences were especially pronounced among those supporting the culture ticket even if not being willing to buy it privately. In fact, everyone who supported the culture ticket without being willing to buy it claimed at least moderate social preferences. More than $90 \%$ of those voting in favor of train or bus ticket in spite of not being willing to buy it privately reported at least moderate social preferences.

Figure 2.3 summarizes these findings. It shows that $77 \%$ to $85 \%$ of all votes can be rationalized by pocketbook voting, corresponding to voting in favor of a ticket 
Table 2.4: Voting in favor but unwilling to buy: importance of social preferences

\begin{tabular}{lccc}
\hline \hline & Train & Bus & Culture \\
\hline Strong social preferences & 137 & 75 & 99 \\
Moderate social preferences & 92 & 69 & 46 \\
No social preferences & 21 & 10 & 0 \\
\hline Total & 250 & 154 & 145 \\
\hline \hline
\end{tabular}

Strong social preferences: at least one social motive was important for the student. Moderate social preferences: at least one social motives was somewhat important for the student but no motive was important. No social preferences: all social motives were unimportant for the student. Social motives considered include for all three tickets savings of other students. They also include environmental aspects for the train ticket, strengthening local public transportation for the bus ticket, and strengthening local cultural life and the belief that others should visit cultural institutions more frequently for the culture ticket.

Figure 2.3: Rationalizing votes - Dataset I

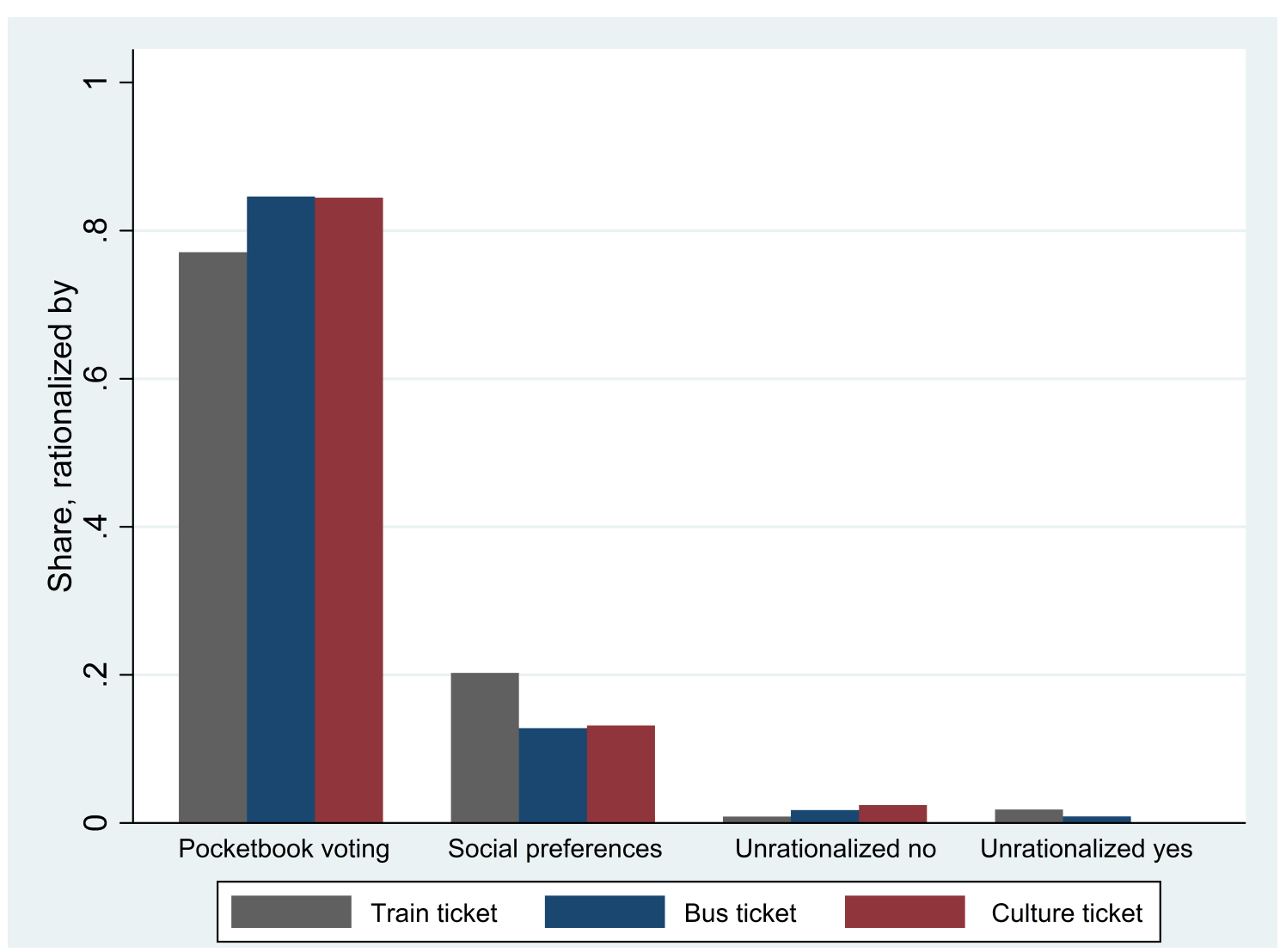


in case one would buy it privately at the price charged and voting against in case one would not buy it. Almost all votes which cannot be rationalized in this way can be rationalized by social preferences. Only one to two percent of respondents voted against a ticket, despite having own pocketbook consideration to support the service. The share of unrationalizable yes votes, those supporting a service which they would not demand privately at the quoted price, and for which they did not express any social concerns, varies between zero and two percent.

When interpreting Figure 2.3, it is important to note that own financial interests and social preferences are not mutually exclusive, but may coincide. Therefore, the figure does not state that $80 \%$ of all voters would base their decision just on their own financial benefit. Rather, it shows that there are very few votes which cannot be rationalized by either pocketbook voting or social considerations or both kinds of motivations together. We have done the analysis summarized in Figure 2.3 splitting the sample by gender and by political orientation. We find that the differences between men and women and between supporters of the left and of the right are minor.

To see the full power of social preferences, note from Table 2.3 that although only a minority of students in the sample would have bought the culture ticket or the bus ticket, a majority supported them in the referenda. As Figure 2.3 shows, for a sizable minority of voters, social preferences were the decisive factor shifting their decision. Given that results in the actual referenda on culture and bus ticket were close, this suggests that social preferences were pivotal in the former vote and came close to being pivotal in the latter.

For each ticket, Table 2.5 goes deeper into various social preferences of those who voted in favor of the ticket but would not buy it privately. For train and bus, altruistic consideration for savings by others is by far the most important social motivation to support the ticket. Four out of five also view environmental benefits as an at least somewhat important motivation to support the train ticket. A collective purchase decision in favor of an environmentally friendlier form of transportation can be seen as a way to avoid free-riding in protecting the environment.

For the culture ticket, a different picture arises. The most common motivation to support this ticket as a voter, even if not being willing to buy it as consumer, 
Table 2.5: Voting in favor but unwilling to buy: different social preferences

\begin{tabular}{lccc|c}
\hline \hline & Important & Somewhat important & Unimportant & Total \\
\hline $\begin{array}{l}\text { Train ticket } \\
\text { Savings of others } \\
\text { Environment }\end{array}$ & 119 & 106 & 29 & 254 \\
$\begin{array}{l}\text { Bus ticket } \\
\text { Savings of others }\end{array}$ & 96 & 105 & 50 & 251 \\
$\begin{array}{l}\text { Strengthening bus system } \\
\text { Culture ticket }\end{array}$ & 63 & 70 & 24 & 157 \\
$\begin{array}{l}\text { Savings of others } \\
\text { Strengthening local culture }\end{array}$ & 54 & 69 & 57 & 155 \\
Others should go & 65 & 65 & 26 & 145 \\
\hline \hline
\end{tabular}

is strengthening local cultural institutions. This motivation, in turn, can have an altruistic component, but also be self-interested: a stronger local cultural landscape improves the choices one has available as private consumer. Altruistic motivation by savings of others and the view that others should attend cultural activities more often are less pronounced. Interestingly, the latter motivation appears to be somewhat more common than concern for the savings of others. Wanting other students to consume more culture can be interpreted as paternalism, but might also reflect a desire to have more company at cultural events.

Yet another reason for supporting the culture ticket could be related to problems of self-control. Students possibly want to commit themselves to consume more culture, just as a flat rate gym membership can be seen as a commitment device to exercise more (DellaVigna and Malmendier, 2006). Note however that buying such a ticket privately would provide a commitment device too. The fact that a substantial number of respondents voting in favor of the ticket would not do this but at the same time state that others should attend cultural events more often suggests that many students see the self-control problem rather in their fellow students than in themselves.

We now turn to Dataset II which refers to the vote in 2010 on the Bahn ticket. First, we note that savings on the trips to parents are highly diverse. While almost half of the students do not use the Bahn ticket at all for visiting their parents, mean savings amount to 255 euros. Figure 2.4 depicts the share of yes votes according to the magnitude of the savings conferred by this ticket for trips to students' parents. 
Figure 2.4: Savings and share of yes votes - Dataset II

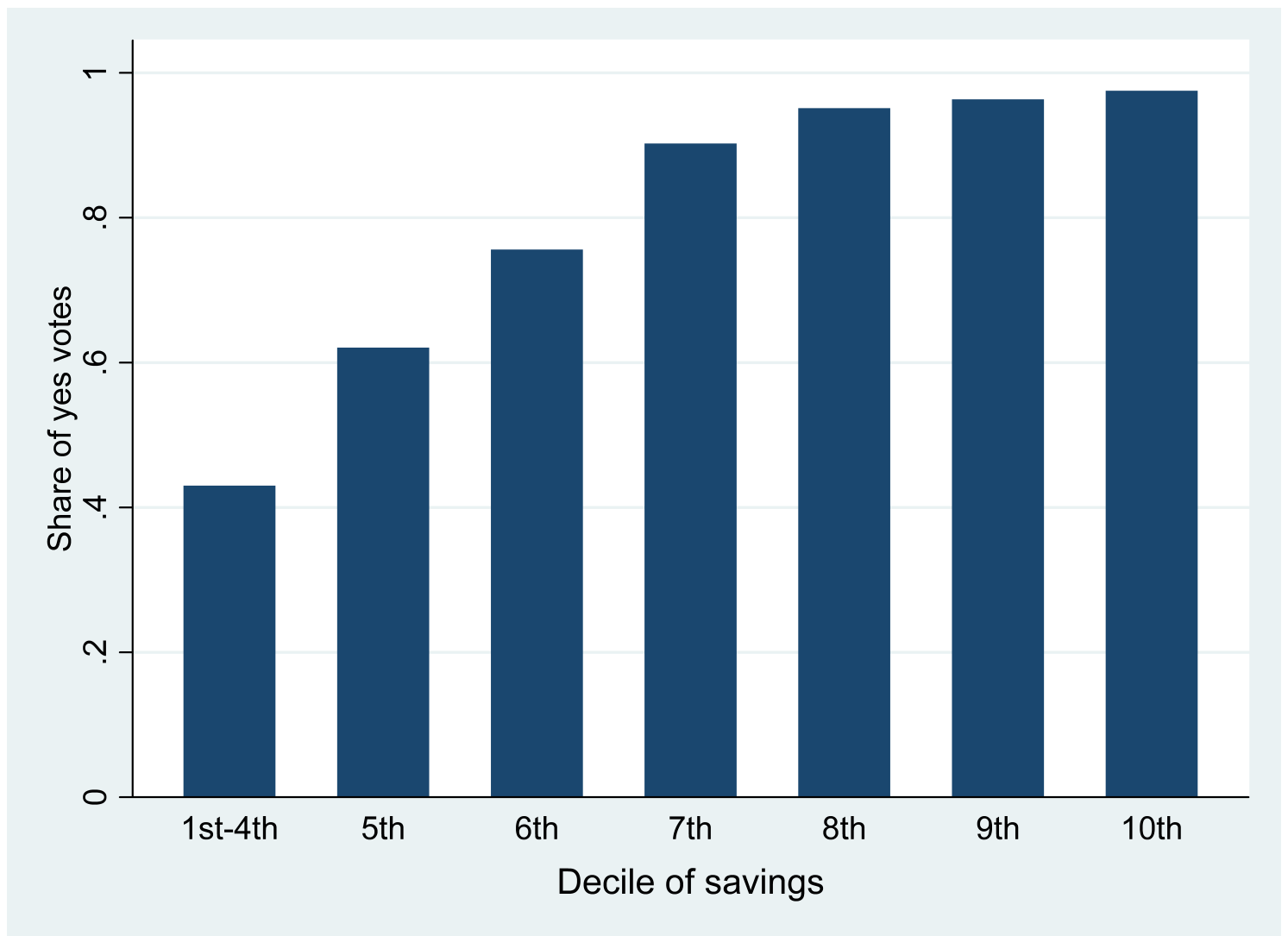

About $40 \%$ in the lowest four deciles, which consist of students with zero savings, voted in favor of the ticket. Thereafter, support for the ticket increased monotonically when moving to higher savings deciles, exceeding $90 \%$ for the four highest deciles. Therefore, the picture we find is again very much in line with pocketbook voting.

We also asked students whether they used the ticket for other visits than those to their parents. This allows us to define those for whom savings from visiting parents were less than the price of the ticket and who did neither visit other people using the ticket nor mentioned leisure or work-related trips as losers in terms of private benefits, and those for whom the savings from visiting parents exceeded the price of the ticket as clear winners. Those for whom savings from visiting parents fell short from the price of the ticket but who also mentioned other trips are a middle 
Table 2.6: Pocketbook voting - Dataset II

\begin{tabular}{|c|c|c|c|}
\hline \multicolumn{4}{|l|}{ Bahn ticket } \\
\hline \multirow{2}{*}{ Net gain } & \multicolumn{2}{|c|}{ Vote } & \multirow[b]{2}{*}{ Total } \\
\hline & No & Yes & \\
\hline Loser & 144 & 49 & 193 \\
\hline Moderate savings \& add. monetary gains & 80 & 140 & 220 \\
\hline Winner & 34 & 368 & 402 \\
\hline Total & 258 & 557 & 815 \\
\hline
\end{tabular}

category, in which we cannot say for sure whether the student in question privately gained or lost from the ticket. Table 2.6 shows that $92 \%$ of winners voted in favor of the ticket, and $75 \%$ of losers against. Therefore, pocketbook voting can again rationalize most of the votes, but there is also a significant minority that voted against their narrowly defined self-interest.

Looking closer at those who voted against their narrowly defined self-interest shows that most respondents who lost privately but voted in favor of the ticket cared about the savings that the ticket delivered to other students. To analyze such concerns, we use the variables altruist $(+)$ and altruist(-) which describe students who stated that they cared about other students' benefits in their vote and at the same time thought that students on average gain or lose, respectively, when the ticket is introduced. As can be seen in Table 2.7, the majority of respondents who voted in favor of the ticket even if they lost privately thought that other students gained from it and reported that they cared about this. Among those who voted against the ticket, even if it promised them higher private savings than the price of the ticket, a clear majority was either of the view that other students would lose from the ticket, or mentioned protest motives regarding price or pricing policy in the questionnaire's write-in section.

We also analyzed how the decision to participate in the vote was related to the savings when visiting parents. Figure 2.5 presents turnout separately for those who lost, for those with moderate savings for whom we cannot say whether other trips than those to visiting parents were enough to make them gain from the ticket 
Table 2.7: Social preferences and protest among winners and losers - Dataset II

\begin{tabular}{c|cc|c}
\hline \hline \multicolumn{4}{c}{ Bahn ticket, only losers } \\
\hline \multirow{2}{*}{ Altruist $(+)$} & \multicolumn{2}{|c}{ Vote } & Total \\
& No & Yes & \\
\hline No & 119 & 17 & 136 \\
Yes & 12 & 23 & 35 \\
\hline Total & 131 & 40 & 171 \\
\hline \hline
\end{tabular}

\begin{tabular}{|c|c|c|c|}
\hline \multicolumn{4}{|c|}{ Bahn ticket, only winners } \\
\hline \multirow{2}{*}{ Altruist $(-)$ or protest } & \multicolumn{2}{|c|}{ Vote } & \multirow{2}{*}{ Total } \\
\hline & No & Yes & \\
\hline No & 9 & 239 & 248 \\
\hline Yes & 23 & 100 & 123 \\
\hline Total & 32 & 339 & 371 \\
\hline
\end{tabular}

Losers' savings do not cover ticket costs and they did neither visit other people using the ticket nor mention leisure/work activities. Winners' savings cover ticket costs.

privately, as well as for those whose savings when visiting parents were bigger than the price. Those with zero and moderate savings are least likely to turn out. After that, turnout increases monotonically. This suggests that those with higher stakes are more likely to vote, in line with rational calculus of voting. It is worth noting that gains and losses are asymmetrically distributed: the maximum loss is the price of ticket (84.48 euros), while the average saving just from visiting parents is among the winners 586 euros.

Taken together, our findings suggest that voters voted on the collective purchasing decisions largely in line with their financial interests. At the same time, social preferences also shaped voting decisions. In particular, there is a considerable altruistic component, and many students explicitly referred to the benefits for other students in the write-in section. A big share of students were also motivated to support public good provision, possibly as a way of solving the free-rider problem in case of private choices: as a result, they supported a collective purchasing decision even at a price for which they would not have been willing to buy the ticket privately. In the area of culture, a paternalistic component is also important, with a large fraction of students being willing to support the culture ticket as a nudge to push other students to use cultural services more often. Differences in turnout decisions are in line with what rational calculus of voting suggests: those who have more to gain are more likely to vote. 
Figure 2.5: Turnout and savings - Dataset II

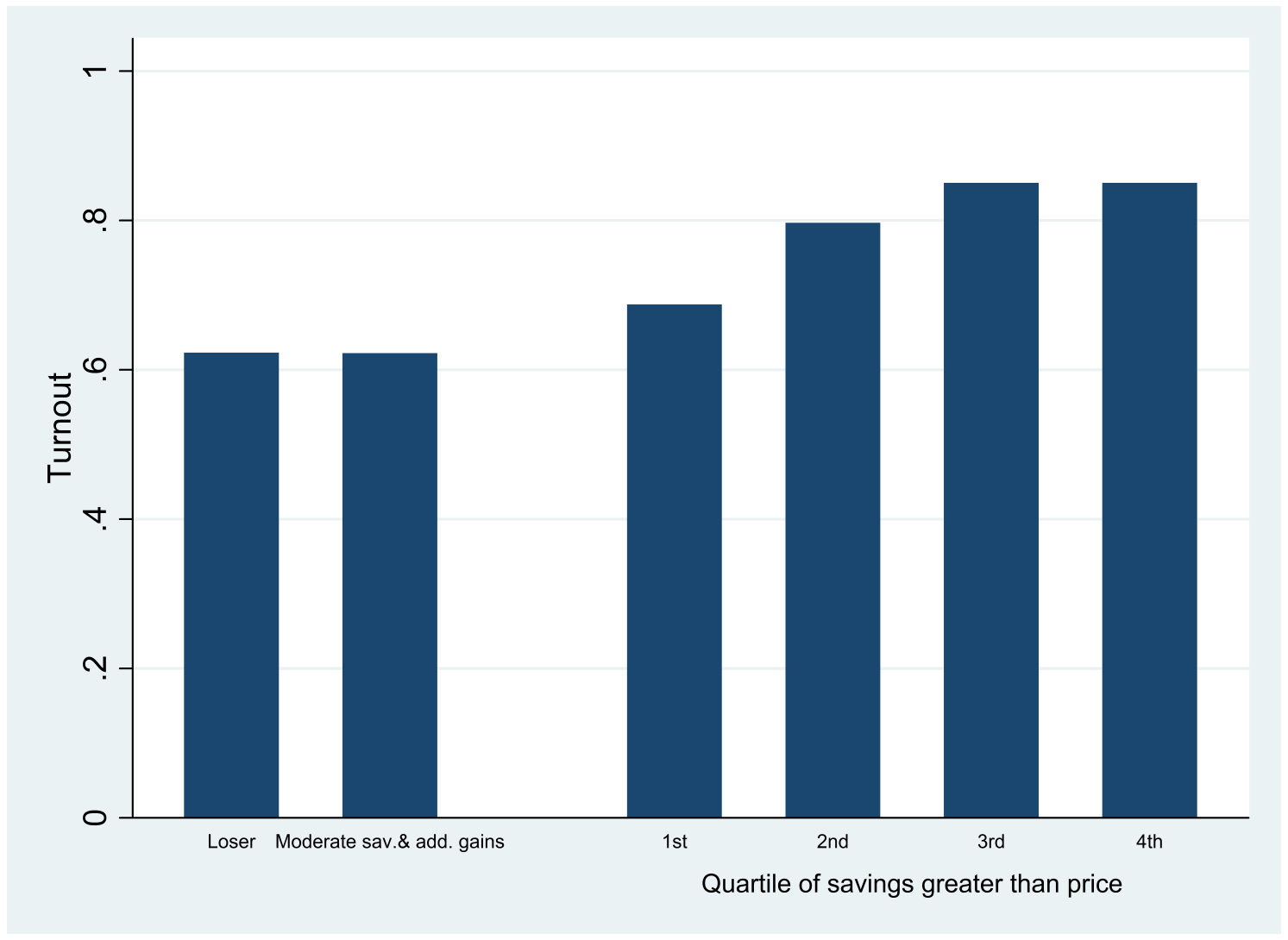

Losers' savings do not cover ticket costs and they did neither visit other people using the ticket nor mention leisure/work activities. Savings of the middle group alone do not cover ticket costs, but they mention other trips.

\subsection{The vote}

We now turn to the regression analysis of the voting decisions. The dependent variable is the probability to vote in favor of the respective ticket which we estimate using probit models. We start with the first dataset and present results for the train, bus and culture ticket in Tables 2.8 to 2.10. Indicator variables for social preferences take the value one if the respondent considered the respective motivation important; descriptive statistics are given in Table 2.1. In order to interpret the results right 
away, we display marginal effects ${ }^{5}$ for benchmark students in the regression tables. These students are characterized by all indicator variables being zero. Thus, the benchmark is male, not a freshman and savings of other students were not important for his decision. The base category of the intensity of use is "never".

Our econometric results confirm the impressions gained in Section 2.3: the probability of voting in favor of a ticket strongly increases in the intensity of personal use, suggesting a high degree of pocketbook voting. The effects are significant at the 0.1 percent-level and of economically relevant size. For instance, concerning the bus ticket estimations imply that an otherwise identical student who uses the bus several times per week is more than 70 percentage-points more likely to vote in favor of this ticket than the benchmark student who does not use the bus.

However, variables capturing social preferences show highly significant and positive effects too. Those who consider savings of other students important for their decision vote in favor of the respective ticket with higher probability. Also, students who consider environmental aspects or strengthening of local public transportation important are more likely to vote in favor of the train ticket or the bus ticket, respectively. The same holds true regarding the culture ticket for those who indicate that strengthening local cultural life or that others should visit these institutions more frequently is important to them in their voting decision. ${ }^{6}$

Freshmen are, ceteris paribus, more likely to vote in favor of the bus ticket and the culture ticket. However, as for freshmen the questions on the intensity of use refer to a shorter period of time or might cover some time when they have not yet been in Goettingen, we refrain from emphasizing this finding.

To examine whether general political attitudes contribute to explaining individual votes, we include party preferences in the regressions. Even though the parties

\footnotetext{
${ }^{5}$ We calculate marginal effects as discrete changes from zero to one for all indicator variables. Coefficients for all regressions are reported in Tables 2.A.1-2.A.6 in Appendix 2.A.III.

${ }^{6} \mathrm{~A}$ very similar picture emerges from the regression analysis if we use indicator variables encompassing motives that were at least somewhat important instead of focusing on motives that were important. In line with expectations, these variables capturing less pronounced social preferences in general display smaller marginal effects than those shown in Tables 2.8-2.10. We have also included complete sets of indicator variables containing information on whether someone considered a motive unimportant, somewhat important, or important, and the conclusions remained the same. (Not reported for brevity.)
} 
Table 2.8: Train ticket - Dataset I

\begin{tabular}{|c|c|c|c|c|}
\hline \multicolumn{5}{|c|}{ Dependent Variable: Supporting Train Ticket } \\
\hline & $(1)$ & $(2)$ & $(3)$ & (4) \\
\hline Rarely ( $\leq 5 /$ year $)$ & $\begin{array}{c}0.428^{* * *} \\
(7.35)\end{array}$ & $\begin{array}{c}0.422^{* * *} \\
(7.41)\end{array}$ & $\begin{array}{c}0.393^{* * *} \\
(6.73)\end{array}$ & $\begin{array}{c}0.373^{* * *} \\
(6.21)\end{array}$ \\
\hline Sometimes (monthly) & $\begin{array}{c}0.657^{* * *} \\
(13.05)\end{array}$ & $\begin{array}{c}0.683^{* * *} \\
(14.23)\end{array}$ & $\begin{array}{c}0.680 * * * \\
(13.56)\end{array}$ & $\begin{array}{c}0.676^{* * * *} \\
(13.14)\end{array}$ \\
\hline Often (weekly) & $\begin{array}{c}0.701^{* * *} \\
(14.10)\end{array}$ & $\begin{array}{c}0.750 * * * \\
(15.65)\end{array}$ & $\begin{array}{c}0.749 * * * \\
(14.61)\end{array}$ & $\begin{array}{c}0.745^{* * *} \\
(14.19)\end{array}$ \\
\hline Very often $(\geq 2 /$ week $)$ & $\begin{array}{c}0.709 * * * \\
(14.39)\end{array}$ & $\begin{array}{c}0.764^{* * *} \\
(16.26)\end{array}$ & $\begin{array}{c}0.766^{* * *} \\
(15.19)\end{array}$ & $\begin{array}{c}0.760 * * * \\
(14.69)\end{array}$ \\
\hline Savings of others & & $\begin{array}{c}0.300^{* * *} \\
(6.11)\end{array}$ & $\begin{array}{c}0.285^{* * *} \\
(5.48)\end{array}$ & $\begin{array}{c}0.198^{* * *} \\
(3.65)\end{array}$ \\
\hline Female & & & $\begin{array}{c}0.090^{*} \\
(2.44)\end{array}$ & $\begin{array}{c}0.074^{*} \\
(2.04)\end{array}$ \\
\hline Freshman & & & $\begin{array}{l}-0.043 \\
(-1.39)\end{array}$ & $\begin{array}{l}-0.029 \\
(-0.92)\end{array}$ \\
\hline Environment & & & & $\begin{array}{c}0.242^{* *} \\
(3.25)\end{array}$ \\
\hline Pseudo $\mathrm{R}^{2}$ & 0.285 & 0.334 & 0.342 & 0.364 \\
\hline Log Likelihood & -356.2 & -315.2 & -298.1 & -277.9 \\
\hline Observations & 1247 & 1217 & 1163 & 1145 \\
\hline
\end{tabular}

Probit estimation, discrete effects for benchmark students from changes from 0 to

1 for all variables, $\mathrm{z}$-statistic in parentheses. ${ }^{*} p<0.05,{ }^{* *} p<0.01,{ }^{* * *} p<0.001$.

Table 2.9: Bus ticket - Dataset I

\begin{tabular}{lcccc}
\hline \multicolumn{4}{c}{ Dependent Variable: Supporting Bus Ticket } \\
\hline Rarely (1 or 2/semester) & $0.241^{* * *}$ & $(2)$ & $(3)$ & $(4)$ \\
& $(6.94)$ & $(6.31)$ & $(6.15)$ & $(5.64)$ \\
Sometimes (monthly) & $0.415^{* * *}$ & $0.398^{* * *}$ & $0.416^{* * *}$ & $0.411^{* * *}$ \\
& $(9.81)$ & $(8.88)$ & $(8.79)$ & $(8.19)$ \\
Often (weekly) & $0.597^{* * *}$ & $0.597^{* * *}$ & $0.617^{* * *}$ & $0.587^{* * *}$ \\
& $(14.88)$ & $(13.63)$ & $(13.58)$ & $(11.38)$ \\
Very often ( $\geq 2 /$ week) & $0.706^{* * *}$ & $0.726^{* * *}$ & $0.744^{* * *}$ & $0.728^{* * *}$ \\
& $(27.55)$ & $(25.34)$ & $(24.39)$ & $(20.17)$ \\
Savings of others & & $0.221^{* * *}$ & $0.204^{* * *}$ & $0.158^{* * *}$ \\
& & $(5.93)$ & $(5.36)$ & $(4.07)$ \\
Female & & -0.006 & 0.007 \\
Freshman & & $(-0.27)$ & $(0.29)$ \\
Strengthening bus system & & $0.081^{* *}$ & $0.067^{*}$ \\
& & & $(2.92)$ & $(2.44)$ \\
Pseudo R & & & & $0.418^{* * *}$ \\
Log Likelihood & & & & $(5.87)$ \\
Observations & 0.224 & 0.245 & 0.252 & 0.285 \\
\hline \hline
\end{tabular}

Probit estimation, discrete effects for benchmark students from changes from 0 to 1 for all variables, z-statistic in parentheses. ${ }^{*} p<0.05,{ }^{* *} p<0.01,{ }^{* * *} p<0.001$. 
Table 2.10: Culture ticket - Dataset I

\begin{tabular}{|c|c|c|c|c|}
\hline \multicolumn{5}{|c|}{ Dependent Variable: Supporting Culture Ticket } \\
\hline & $(1)$ & $(2)$ & $(3)$ & $(4)$ \\
\hline Rarely (1 or $2 /$ year) & $\begin{array}{c}0.376^{* * *} \\
(11.67)\end{array}$ & $\begin{array}{c}0.347^{* * *} \\
(9.91)\end{array}$ & $\begin{array}{c}0.355^{* * *} \\
(9.60)\end{array}$ & $\begin{array}{c}0.290^{* * *} \\
(6.83)\end{array}$ \\
\hline Sometimes (3 to $5 /$ year) & $\begin{array}{c}0.578^{* * *} \\
(18.57)\end{array}$ & $\begin{array}{c}0.570^{* * *} \\
(15.26)\end{array}$ & $\begin{array}{c}0.597 * * * \\
(13.28)\end{array}$ & $\begin{array}{c}0.572^{* * *} \\
(7.80)\end{array}$ \\
\hline Often (6 to $10 /$ year) & $\begin{array}{c}0.634^{* * *} \\
(17.78)\end{array}$ & $\begin{array}{c}0.643^{* * *} \\
(14.19)\end{array}$ & $\begin{array}{c}0.693 * * * \\
(12.09)\end{array}$ & $\begin{array}{c}0.649 * * * \\
(4.94)\end{array}$ \\
\hline Very often $(>10 /$ year $)$ & $\begin{array}{c}0.605^{* * * *} \\
(13.59)\end{array}$ & $\begin{array}{c}0.585^{* * * *} \\
(9.37)\end{array}$ & $\begin{array}{c}0.640^{* * *} \\
(7.96)\end{array}$ & $\begin{array}{c}0.522 * * \\
(2.95)\end{array}$ \\
\hline Savings of others & & $\begin{array}{c}0.280^{* * * *} \\
(6.91)\end{array}$ & $\begin{array}{c}0.262^{* * *} \\
(6.21)\end{array}$ & $\begin{array}{c}0.104^{*} \\
(2.40)\end{array}$ \\
\hline Female & & & $\begin{array}{c}0.071^{*} \\
(2.53)\end{array}$ & $\begin{array}{l}0.019 \\
(0.80)\end{array}$ \\
\hline Freshman & & & $\begin{array}{c}0.109 * * \\
(3.17)\end{array}$ & $\begin{array}{c}0.084^{*} \\
(2.58)\end{array}$ \\
\hline Others should go & & & & $\begin{array}{c}0.433^{* * *} \\
(5.83)\end{array}$ \\
\hline Strengthening local culture & & & & $\begin{array}{c}0.453^{* * *} \\
(8.74)\end{array}$ \\
\hline Pseudo $\mathrm{R}^{2}$ & 0.192 & 0.215 & 0.215 & 0.440 \\
\hline Log Likelihood & -664.7 & -599.2 & -571.1 & -378.7 \\
\hline Observations & 1189 & 1110 & 1055 & 988 \\
\hline
\end{tabular}

traditionally present in German parliaments cannot easily be ordered in a strict way from the left to the right, there arguably exists the general consensus that Social Democrats and the Greens represent the center left and that the Left Party is positioned according to its name. Liberal Democrats and Christian Democrats form the center right.

As is apparent from Table 2.A.7 in Appendix 2.A.IV, the strong empirical support for both pocketbook voting and social considerations is robust to the inclusion of party preferences. Furthermore, for both the train and the bus tickets, we find almost no significant effects for any of the parties. ${ }^{7}$ Thus, party politics in general is not driving the voting decisions here. ${ }^{8}$ However, political attitudes seem to play a role when it comes to the joint provision of local cultural goods. Even within the left bloc our results show differences with respect to voting behavior. Namely, we find

\footnotetext{
${ }^{7}$ The only exception are negative effects of Liberal Democrats, which are significant at 10 or 5 percent-levels in Tables 2.A.7 and 2.A.9 respectively.

${ }^{8}$ Results are similar if we group parties to the left bloc and the right bloc.
} 
that support for the culture ticket, ceteris paribus, increases as party preferences become more leftist.

As a robustness check, we also control for the fields of study (Table 2.A.8 in Appendix 2.A.IV). The overall picture is that they do not seem to matter much for individual votes. Only a small number of fields show significant effects on the voting decisions and our main results remain robust. All else being equal, students of the humanities and social sciences are more likely to vote in favor of the culture ticket. One explanation for this can be based on the large share of students in these fields that study culture-related subjects. Our main findings are also robust to the inclusion of both fields and party preferences into the regressions (Table 2.A.9 in Appendix 2.A.IV).

Turning to our second dataset and the constructed measure of monetary savings, a very similar picture emerges. In order to account for the expected non-linear effect of savings on the decision we use the natural logarithm of savings, after adding 1 euro, in the regressions. Table 2.11 shows corresponding marginal effects for benchmark students. Again, the benchmark is defined by all indicator variables being zero. However, to account for the high variation with respect to individual savings on trips to parents, we incorporate the individual values of the savings variable also in the calculation of marginal effects. Hence, we display average marginal effects for benchmark students.

The main variable of interest, the natural logarithm of savings on trips to parents, is significant at the 0.1 percent-level and shows the expected positive sign. The corresponding marginal effect does virtually not change if we include additional variables. It implies that a benchmark student is on average $0.7-0.8$ percentage points more likely to vote in favor if savings increase by $10 \%$. Given the range of the variable, this translates into sizeable differences in the prediction: Based on the full specification, column (5), the probability of a positive vote is $14 \%$ for a benchmark student who does not save at all on trips to parents. This value increases to $56 \%$ if his savings just cover ticket costs, which is remarkably close to our expectations based on theoretical considerations; such a student should be fairly indifferent between the alternatives. The predicted probability is $67 \%$ if his savings are of average size and $79 \%$ if he saved a thousand euros. In addition, visiting others using the ticket 
Table 2.11: Bahn ticket - Dataset II

\begin{tabular}{|c|c|c|c|c|c|}
\hline \multicolumn{6}{|c|}{ Dependent Variable: Supporting Bahn Ticket } \\
\hline & $(1)$ & $(2)$ & $(3)$ & $(4)$ & (5) \\
\hline Log savings & $\begin{array}{c}0.070^{* * *} \\
(24.47)\end{array}$ & $\begin{array}{c}0.070^{* * *} \\
(17.90)\end{array}$ & $\begin{array}{c}0.073^{* * *} \\
(18.36)\end{array}$ & $\begin{array}{c}0.077^{* * *} \\
(22.52)\end{array}$ & $\begin{array}{c}0.078^{* * * *} \\
(24.48)\end{array}$ \\
\hline Leisure/work & & $\begin{array}{c}0.079 \\
(1.02)\end{array}$ & $\begin{array}{l}0.033 \\
(0.41)\end{array}$ & $\begin{array}{l}0.042 \\
(0.52)\end{array}$ & $\begin{array}{l}0.046 \\
(0.56)\end{array}$ \\
\hline Visiting others & & $\begin{array}{c}0.296^{* * *} \\
(8.31)\end{array}$ & $\begin{array}{c}0.308^{* * *} \\
(8.22)\end{array}$ & $\begin{array}{c}0.269^{* * *} \\
(6.50)\end{array}$ & $\begin{array}{c}0.272^{* * * *} \\
(6.62)\end{array}$ \\
\hline Female & & & $\begin{array}{l}0.055 \\
(1.51)\end{array}$ & $\begin{array}{l}0.043 \\
(1.13)\end{array}$ & $\begin{array}{l}0.042 \\
(1.09)\end{array}$ \\
\hline Freshman & & & $\begin{array}{l}0.103^{*} \\
(2.04)\end{array}$ & $\begin{array}{c}0.127^{*} \\
(2.42)\end{array}$ & $\begin{array}{l}0.134^{*} \\
(2.57)\end{array}$ \\
\hline Altruist $(-)$ & & & & $\begin{array}{c}-0.149^{* *} \\
(-3.04)\end{array}$ & $\begin{array}{c}-0.147^{* *} \\
(-2.94)\end{array}$ \\
\hline Altruist $(+)$ & & & & $\begin{array}{c}0.288^{* * *} \\
(6.78)\end{array}$ & $\begin{array}{c}0.286^{* * *} \\
(6.83)\end{array}$ \\
\hline Protest & & & & & $\begin{array}{l}-0.080 \\
(-1.93) \\
\end{array}$ \\
\hline Pseudo $\mathrm{R}^{2}$ & 0.225 & 0.306 & 0.333 & 0.434 & 0.438 \\
\hline Log Likelihood & -395.7 & -351.7 & -307.4 & -236.0 & -234.2 \\
\hline Observations & 818 & 810 & 741 & 669 & 669 \\
\hline
\end{tabular}

Probit estimation, marginal effects for benchmark students, discrete changes from 0 to 1 for indicator variables, $\mathrm{z}$-statistic in parentheses. ${ }^{*} p<0.05,{ }^{* *} p<0.01,{ }^{* * *} p<0.001$.

significantly increases the probability of voting in favor. This confirms the high importance of personal monetary benefits for the individual vote.

However, social preferences have their share in this vote too: both altruism variables carry the expected sign and are significant at least at the 1 percent-level. About half of the students consider their fellow students' gains and losses in their vote. According to their own perception of whether the other students on average gain or lose, these students are, ceteris paribus, more or less likely, respectively, than the benchmark to vote in favor of the ticket. Expecting other students to gain from the ticket and considering this, increases support for the ticket as much as using it oneself to also visit other people than one's parents. Furthermore, the protest variable carries a negative sign and is almost significant at the 5 percent-level. So, there is weak evidence suggesting that some students expressed their protest against the train company's pricing policy by voting against the ticket.

Finally, as in the analysis of Dataset I, including general political preferences in the regressions does not change our main results. All else being equal, supporters of the left are not more likely to vote in favor of the ticket; we do not find significant 
effects for any of the parties (Table 2.A.10 in Appendix 2.A.IV) ${ }^{9}$

Without putting too much emphasis on this, it is noted that especially private savings and the altruism variables contribute to the pseudo R-squared in Table 2.11.

A general concern related to survey data is how reliable answers are. In our setting, this problem may arise in particular when it comes to social preferences because of social desirability. For example, one might wonder whether respondents genuinely care for others or just feel social pressure to express such a concern. However, both the descriptive and econometric analyses show that stated social preferences, in particular altruism, have a substantial impact on the voting decision, explaining most votes which cannot be rationalized by monetary benefits.

From an econometric perspective, one might be concerned with reverse causality, omitted variables or sample selection. A reverse causality problem would arise if those respondents who voted in favor of a ticket against their monetary interest ex post rationalized their decision by mentioning social preferences. However, this argumentation leaves open the question of why they voted in favor of the ticket in the first place. Given the extensive set of controls we use, there is no obvious candidate for an omitted variable which affects the voting decision and is correlated with the explanatory variables. An issue of sample selection could arise if respondents in the surveys systematically differed from the student population. As shown in Section 2.2, voters are somewhat overrepresented in Dataset II. This is, however, not a problem since we excluded nonvoters from the analysis of voting decisions. Finally, semester tickets should only play a minor role when deciding whether to enroll at Goettingen University. Taken together, we do not think that any of these issues is likely to seriously bias our results.

Summarizing our empirical analysis so far, we find, first, that pocketbook voting is an important determinant of referendum outcomes, second, that party politics plays only a minor role and, third, that monetary self-interest is not the whole story. In particular, social preferences should not be disregarded. Especially so, as the descriptive analysis in Section 2.3 suggested that these motives were or came close to being pivotal in two out of four referenda studied.

\footnotetext{
${ }^{9}$ This holds true also for grouping of the left and right into blocs.
} 


\subsection{Participation}

We now turn to the second part of our analysis, which is to understand what induced students to turn out in the referendum. For this purpose, we use Dataset II which also contains detailed information on non-voters.

Specifically, we investigate whether the calculated savings are also able to explain participation in the referendum. Hence, we estimate the probability of taking part in the vote conditional on the explanatory variables using probit specifications. As described above, we therefore transform savings into stakes, defined as the absolute value of the difference of the yearly price and savings. In the regressions, we use the natural logarithm of these stakes augmented by one euro. Table 2.12 shows average marginal effects for benchmark students. ${ }^{10}$ Looking at the full sample, regressions (1)-(4), we see a highly significant positive effect of stakes. This effect is robust to the inclusion of additional control variables. It seems that students whose stakes are high make sure to take part in the referendum.

Table 2.12: Taking part - Dataset II

\begin{tabular}{lcccccc}
\hline \hline \multicolumn{7}{c}{ Dependent Variable: Taking Part in Referendum } \\
\hline & $(1)$ & $(2)$ & $(3)$ & $(4)$ & $(5)$ & $(6)$ \\
& all & all & all & all & savings $\geq$ price & savings < price \\
\hline Log stakes & $0.070^{* * *}$ & $0.072^{* * *}$ & $0.074^{* * *}$ & $0.077^{* * *}$ & $0.046^{* *}$ & 0.041 \\
& $(5.94)$ & $(5.85)$ & $(5.88)$ & $(6.00)$ & $(2.99)$ & $(1.04)$ \\
Leisure/work & & -0.017 & -0.007 & -0.008 & -0.014 & -0.003 \\
& & $(-0.28)$ & $(-0.12)$ & $(-0.14)$ & $(-0.16)$ & $(-0.03)$ \\
Visiting others & & 0.041 & 0.033 & 0.031 & 0.036 & -0.009 \\
& & $(1.46)$ & $(1.17)$ & $(1.09)$ & $(0.73)$ & $(-0.22)$ \\
Female & & & $-0.106^{* * *}$ & $-0.107^{* * *}$ & $-0.124^{* *}$ & $-0.087^{*}$ \\
& & & $(-3.61)$ & $(-3.58)$ & $(-2.61)$ & $(-2.16)$ \\
Freshman & & & $0.137^{* * *}$ & $0.140^{* * *}$ & 0.081 & $0.188^{* * *}$ \\
& & & $(4.29)$ & $(4.18)$ & $(1.85)$ & $(3.95)$ \\
Protest & & & & $0.087^{* *}$ & 0.061 & $0.094^{*}$ \\
& & & & $(2.81)$ & $(1.51)$ & $(2.09)$ \\
\hline Pseudo $\mathrm{R}^{2}$ & 0.023 & 0.026 & 0.050 & 0.056 & 0.046 & 0.027 \\
Log Likelihood & -713.1 & -700.8 & -625.6 & -622.0 & -213.2 & -403.5 \\
Observations & 1189 & 1174 & 1075 & 1075 & 449 & 626 \\
\hline \hline
\end{tabular}

Probit estimation, marginal effects for benchmark students, discrete changes from 0 to 1 for indicator variables, z-statistic in parentheses. Regression (5): only students whose savings cover ticket costs (winners), regression (6): only students whose savings do not cover ticket costs. ${ }^{*} p<0.05,{ }^{* *} p<0.01,{ }^{* * *} p<0.001$

\footnotetext{
${ }^{10} \mathrm{As}$ in Section 2.4 all indicator variables are zero for benchmark students. The continuous variable, $\log$ of stakes plus 1 euro, enters calculations of marginal effects at individual values.
} 
Unlike for the decision to vote in favor or against, visiting others does not have a significant effect on the decision to take part. Being female reduces the probability of voting, whereas being freshman increases it. However, we cannot disentangle alternative possible explanations driving the freshman effect. It could be based on the fact that savings of freshmen refer to a shorter period of time, or alternatively, it may reflect that many freshmen are more easily reached by voting advertisements or more enthusiastic to use the first chance to take part in such a referendum. Students who mention protest motives go to the polls with a significantly higher probability.

Splitting the sample into those whose savings on trips to parents cover ticket costs and those whose savings do not, we find that stakes remain significant among the winners at the 1 percent-level, but turn insignificant among the second group. This may be due to the asymmetric distribution of gains and losses: the latter are limited to the yearly price of the ticket, 84.48 euros, whereas stakes of someone who uses the Bahn ticket every weekend to visit his or her parents can be much higher.

Remarkably, those who mention protest motives take part in the referendum with significantly higher probability only among those whose savings fall short of the ticket price. One potential interpretation for this result could be that for someone who gains monetarily from the ticket, protest and monetary interest are opposing motives. Contrary to that, in the case of smaller savings, both motives should shift the vote in the direction of rejection of the ticket. Therefore, the latter group might have a stronger opinion concerning rejection or approval, which could foster participation.

In order to further examine the question whether high gains drive our results here, we gradually remove observations with the highest stakes from the dataset. Table 2.13 contains corresponding average marginal effects for benchmark students. In regression (1), we only leave out the top $5 \%$ students in terms of stakes, whereas in regression (6), we only use the bottom $70 \%$. Stakes have a positive and highly significant effect if we use almost all observations. The more we remove observations with high stakes, the smaller the size and z-statistic of the marginal effect become. Looking at the bottom $75 \%$ only, the effect is not significantly different from zero anymore. In contrast, the significance of the control variables female, freshman and protest suffers much less from this reduction of the sample, and the marginal 
Table 2.13: Taking part, reduced sample - Dataset II

\begin{tabular}{|c|c|c|c|c|c|c|}
\hline \multicolumn{7}{|c|}{ Dependent Variable: Taking Part in Referendum } \\
\hline \multirow[b]{2}{*}{ Bottom } & $(1)$ & $(2)$ & $(3)$ & $(4)$ & $(5)$ & $(6)$ \\
\hline & $95 \%$ & $90 \%$ & $85 \%$ & $80 \%$ & $75 \%$ & $70 \%$ \\
\hline \multirow[t]{2}{*}{ Log stakes } & $0.082^{* * *}$ & $0.073^{* * *}$ & $0.068^{* * *}$ & $0.052^{*}$ & 0.029 & 0.011 \\
\hline & $(5.32)$ & $(4.21)$ & $(3.41)$ & $(2.23)$ & $(1.09)$ & $(0.39)$ \\
\hline \multirow[t]{2}{*}{ Leisure/work } & -0.006 & -0.012 & -0.022 & -0.011 & -0.005 & -0.028 \\
\hline & $(-0.10)$ & $(-0.17)$ & $(-0.31)$ & $(-0.15)$ & $(-0.07)$ & $(-0.35)$ \\
\hline \multirow{2}{*}{ Visiting others } & 0.037 & 0.038 & 0.043 & 0.037 & 0.023 & 0.007 \\
\hline & $(1.26)$ & $(1.25)$ & $(1.35)$ & $(1.11)$ & $(0.67)$ & $(0.19)$ \\
\hline \multirow[t]{2}{*}{ Female } & $-0.106 * * *$ & $-0.106^{* *}$ & $-0.102^{* *}$ & $-0.094^{* *}$ & $-0.097^{* *}$ & $-0.081^{*}$ \\
\hline & $(-3.39)$ & $(-3.27)$ & $(-3.07)$ & $(-2.72)$ & $(-2.74)$ & $(-2.22)$ \\
\hline \multirow[t]{2}{*}{ Freshman } & $0.150^{* * *}$ & $0.153^{* * *}$ & $0.148^{* * *}$ & $0.162^{* * *}$ & $0.160 * * *$ & $0.157^{* * *}$ \\
\hline & $(4.15)$ & $(4.08)$ & $(3.71)$ & $(3.86)$ & $(3.63)$ & $(3.39)$ \\
\hline \multirow{2}{*}{ Protest } & $0.098 * *$ & $0.100 * *$ & $0.098 * *$ & $0.099 * *$ & $0.109 * *$ & $0.105^{* *}$ \\
\hline & $(3.01)$ & $(3.00)$ & $(2.76)$ & $(2.67)$ & $(2.88)$ & $(2.69)$ \\
\hline Pseudo $\mathrm{R}^{2}$ & 0.052 & 0.044 & 0.036 & 0.030 & 0.027 & 0.022 \\
\hline Log Likelihood & -594.8 & -580.7 & -558.1 & -535.0 & -509.4 & -486.7 \\
\hline Observations & 1011 & 967 & 912 & 858 & 806 & 761 \\
\hline
\end{tabular}

Probit estimation, marginal effects for benchmark students, discrete changes from 0 to 1 for indicator variables, z-statistic in parentheses. Columns show percentiles with respect to stakes, e.g. column (1) contains those observations who belong to the bottom $95 \%$ with respect to stakes. ${ }^{*} p<0.05,{ }^{* *} p<0.01,{ }^{* * *} p<0.001$.

effects are much more stable. This suggests that the loss of significance of the stakes variable should not be attributed to the smaller sample size alone.

Reverse causality should not be a problem in the analysis of the participation decision. Variables capturing travel patterns and demographics are clearly not affected by the decision to vote. The protest variable is derived from the write-in section, so that it seems unlikely that it captures ex-post rationalization of participation decision. In contrast, stated altruism could be affected by the participation decision. In fact, non-voters more often claim that they would have considered the benefit of others, had they voted, than voters do. One interpretation for this is that, given that one did not take an actual decision, it is easy to claim noble motivations. For this reason, we did not include the altruism variables in the regressions explaining the participation decision.

Sample selection and omitted variables are a more serious concern given the underrepresentation of non-voters in the sample. If the decisions to vote and to take part in the survey are affected by the same unobserved variable, our participation regressions could be biased. However, it is not clear what such a variable could be 
and in which direction it would bias our results.

Summing up, the results from this section shed some light on the motives to turn out in referenda. First, we find that students who mention protest motives take part with a higher probability, suggesting expressive voting. Second, our results are also in line with the theory of instrumental voting which predicts that for a given probability of being pivotal and given costs of voting, participation should increase in stakes. More specifically, we conclude that especially those who gain a lot, and hence, lose a lot if the ticket fails, drive this result. Thus, in a referendum, one may expect a disproportionately higher turnout by voters who stand to benefit substantially when the proposal passes, whereas voters who are affected only moderately are more likely to abstain.

\subsection{Conclusion}

We investigate determinants of individual votes in four referenda on deep-discount flat rate tickets on train, bus and cultural services held among university students. Introducing such a ticket resembles the collective provision of a public good. The service becomes much cheaper by providing it collectively, but all voters, including those who do not use the service, have to pay taxes to finance it.

Our results show that monetary interests are a major driver of both turnout and voting decisions. However, we also find that in addition to this 'pocketbook voting', altruistic and social motives such as the costs and benefits of other students or a desire to support local public transportation or cultural life are also important, and occasionally even decisive for the referendum outcome. Finally, we found evidence for some students taking part in the referendum in order to express dissatisfaction with the train company's pricing policy. Remarkably, among those upset by the high price of the ticket, those who would lose from the ticket being introduced were more likely to turn out to express their protest.

The set of referenda we study concerns a relatively small group of voters and has the specific feature that voters had very good information on individual costs and benefits of the decision on the ballot. While this allows to study voting motives in a clearly defined setting, it remains an open question to study to what extent our 
results carry over to referenda in a broader context. In particular, we expect ideology and general political attitudes to play a larger role when it comes to referenda on much bigger issues. Nevertheless, similarly to laboratory experiments, it seems plausible that the major voting motives we identify in the present study will also be active in other direct democratic decisions. 


\section{Appendix to Chapter 2}

\section{Appendix 2.A.I: Data handling}

The survey for Dataset II was conducted online between July and November 2010. It was advertised in lectures, on posters on campus and in two e-mails that were sent to all students of Goettingen University from the office of student affairs. On the survey webpage students were informed that the survey was conducted for research purposes and about a possibility to participate in a lottery. At the end of the questionnaire, students were provided with a link to another webpage, where they could register for the lottery. In order to identify winners without ambiguity, we required the (unique) student ID number for a registration for the lottery as well as an e-mail address. For the sake of data protection, questionnaire data and lottery data were collected in separate databases. As the savings variable plays a key role in explaining the decision on whether to vote and if voting, then how to vote, we excluded observations without information on voting behavior or for which we cannot assess savings, because, for instance, we lack data on traveling behavior or the corresponding student started studying only one month before the referendum took place. Besides, we removed less than fifteen observations from the dataset due to clear data errors or where answers given seemed highly implausible, such as visiting parents 20,000 times in one year.

A challenge in the data management is that 75 ID numbers show up twice and on different days, suggesting that 75 students also show up twice in the dataset. Using the time stamps of the lottery data showed that in most cases, the responses were entered shortly after receiving an e-mail from the office of student affairs that was advertising the survey. Therefore, it is likely that these students had forgot that they had already answered a survey or thought that they should answer for a second time. After we explored this issue, we realized that we also have time stamps for survey responses, although in a separate data base. To guarantee the anonymity of respondents, we recruited a research assistant who was not otherwise connected to the project to select those entries from the lottery data that belong to duplicates in the lottery data base and to provide the two timestamps for each of the 75 pairs. 
As only timestamps were extracted pairwisely from the lottery database, anonymity was guaranteed at all times. Specifically, no individual information that could be used to identify the person behind a timestamp, such as a student ID number, was extracted from the lottery data.

Timestamps from the lottery were then assigned to the time stamps in the response dataset. For all pairs of time stamps, we examined responses that were submitted close to the time stamp in the lottery. For all pairs of these subsamples, we identified potential pairs of observations present in both subsamples, based on the data provided. Initial selection was made using gender, year of birth and the zip code of their parents. If students indicated that their parents do not live together, the maternal, or if missing the paternal, zip code was used. If these three variables, year of birth, gender and parental zip code, were the same for observations in both subsamples, they were considered potential duplicates based on the data provided. Potential duplicates were then compared based on additional variables, such as subjects studied, travel frequencies, general attitudes and voting decisions, party preferences and reasons to be in favor and against the ticket. This procedure allowed us to identify 46 pairs of assigned duplicates. Consequently, we dropped the later entry of every pair of duplicates from the dataset. This left us with 29 pairs of duplicates which we could not identify. This corresponds to about two percent of the dataset and should, therefore, have only a tiny effect on the results. To be on the safe side, we also replicated the analysis of the second dataset without excluding observations based on this assignment. The results remain virtually unchanged.

\section{Appendix 2.A.II: Construction of the savings variable}

In the survey, students were asked about their parents' address and how many times they visited their parents' residence within the last 12 months (July 1, 2009 - June 30, 2010) using the Bahn ticket. If students indicated that their parents did not live in the same city, questions were asked for both parents separately.

To translate trips to parents into monetary savings, the nearest train station covered by the Bahn ticket was identified for every parental address (zip code) using 
a standard route planner. ${ }^{11}$ Afterwards, for each station, the relevant price was derived. Therefore, we identified the suggested route to Goettingen using local trains for all stations on the Bahn tracks (dashed blue lines on the map, Figure 2.1) using software provided by Deutsche Bahn. At the time of the referendum, it was already known that students could use the MetroCan tracks (solid red lines) without additional costs. Consequently, savings per trip from the Bahn ticket are the price that would have to be paid to the station on this route where the "free" train (red) is entered. ${ }^{12}$

If the determined price was greater than 21 euros it was capped to this amount to reflect the possibility to buy the so called Lower-Saxony-ticket that is valid on all local trains in the state on the day of validation at this price. As furthermore every visit consists of the way back and forth, final savings are calculated as the product of the relevant price and twice the number of visits using the Bahn ticket. To also reflect the opportunity to buy a train ticket for all trains in Germany including high speed trains (BahnCard100) at a price of 3800 euros per year at the time of the survey, the savings variable is capped at 3800 euros.

For some students in Dataset II, Goettingen is not the nearest train station. Most of these students live in the same town as their parents, presumably with their parents. For these students, the savings variable captures direct monetary savings when they come to campus. If they live apart from their parents we calculate savings correspondingly, starting from their respective nearest station. Ten students, however, live outside Goettingen apart from their parents and save little on trips to them, but could primarily use this ticket for commuting. We refrain from calculating savings in these cases as they might severely misrepresent the benefit from the ticket. Consequently, these observations are dropped.

\footnotetext{
${ }^{11}$ Google maps, standard proposal for cars.

${ }^{12}$ Due to the non-linear pricing in the German railway market, this price is in most cases not equal to the price from a station to Goettingen less the price from the station where the free train is entered to Goettingen.
} 


\section{Appendix 2.A.III: Coefficients}

Table 2.A.1: Train ticket - Dataset I, coefficients for Table 2.8

\begin{tabular}{lcccc}
\hline \hline \multicolumn{4}{c}{ Dependent Variable: Supporting Train Ticket } \\
\hline & $(1)$ & $(2)$ & $(3)$ & $(4)$ \\
\hline Rarely ( $\leq 5 /$ year $)$ & $1.131^{* * *}$ & $1.164^{* * *}$ & $1.099^{* * *}$ & $1.060^{* * *}$ \\
& $(0.17)$ & $(0.19)$ & $(0.19)$ & $(0.20)$ \\
Sometimes (monthly) & $2.047^{* * *}$ & $2.018^{* * *}$ & $1.997^{* * *}$ & $1.976^{* * *}$ \\
& $(0.18)$ & $(0.19)$ & $(0.20)$ & $(0.21)$ \\
Often (weekly) & $2.452^{* * *}$ & $2.423^{* * *}$ & $2.386^{* * *}$ & $2.334^{* * *}$ \\
& $(0.21)$ & $(0.22)$ & $(0.23)$ & $(0.24)$ \\
Very often ( $\geq 2 /$ week) & $2.574^{* * *}$ & $2.555^{* * *}$ & $2.528^{* * *}$ & $2.439^{* * *}$ \\
& $(0.21)$ & $(0.23)$ & $(0.24)$ & $(0.24)$ \\
Savings of others & & $0.855^{* * *}$ & $0.826^{* * *}$ & $0.613^{* * *}$ \\
& & $(0.13)$ & $(0.13)$ & $(0.15)$ \\
Female & & & $0.301^{* *}$ & $0.260^{*}$ \\
& & & $(0.12)$ & $(0.12)$ \\
Freshman & & & -0.179 & -0.122 \\
& & & $(0.12)$ & $(0.13)$ \\
Environment & & & & $0.730^{* * *}$ \\
& & & $(0.18)$ \\
Constant & $-0.628^{* * *}$ & $-0.883^{* * *}$ & $-0.912^{* * *}$ & $-0.947^{* * *}$ \\
& $(0.15)$ & $(0.17)$ & $(0.19)$ & $(0.20)$ \\
\hline Pseudo $\mathrm{R}^{2}$ & 0.285 & 0.334 & 0.342 & 0.364 \\
Log Likelihood & -356.2 & -315.2 & -298.1 & -277.9 \\
Observations & 1247 & 1217 & 1163 & 1145 \\
\hline \hline
\end{tabular}

Probit estimation, coefficients, standard errors in parentheses. ${ }^{*} p<0.05,{ }^{* *} p<0.01,{ }^{* * *} p<0.001$. 
Table 2.A.2: Bus ticket - Dataset I, coefficients for Table 2.9

\begin{tabular}{|c|c|c|c|c|}
\hline \multicolumn{5}{|c|}{ Dependent Variable: Supporting Bus Ticket } \\
\hline & (1) & $(2)$ & $(3)$ & $(4)$ \\
\hline Rarely ( 1 or $2 /$ semester) & $\begin{array}{c}0.659^{* * *} \\
(0.10)\end{array}$ & $\begin{array}{c}0.630^{* * *} \\
(0.10)\end{array}$ & $\begin{array}{c}0.654^{* * *} \\
(0.10)\end{array}$ & $\begin{array}{c}0.631^{* * *} \\
(0.11)\end{array}$ \\
\hline Sometimes (monthly) & $\begin{array}{c}1.106^{* * *} \\
(0.12)\end{array}$ & $\begin{array}{c}1.086^{* * *} \\
(0.12)\end{array}$ & $\begin{array}{c}1.159^{* * * *} \\
(0.13)\end{array}$ & $\begin{array}{c}1.160^{* * *} \\
(0.14)\end{array}$ \\
\hline Often (weekly) & $\begin{array}{c}1.690^{* * *} * \\
(0.16)\end{array}$ & $\begin{array}{c}1.674^{* * *} \\
(0.16)\end{array}$ & $\begin{array}{c}1.746^{* * *} \\
(0.16)\end{array}$ & $\begin{array}{c}1.653^{* * *} \\
(0.17)\end{array}$ \\
\hline Very often $(\geq 2 /$ week $)$ & $\begin{array}{c}2.306^{* * *} \\
(0.16)\end{array}$ & $\begin{array}{c}2.305^{* * *} \\
(0.17)\end{array}$ & $\begin{array}{c}2.350^{* * *} \\
(0.18)\end{array}$ & $\begin{array}{c}2.222^{* * *} \\
(0.19)\end{array}$ \\
\hline Savings of others & & $\begin{array}{c}0.637^{* * *} \\
(0.10)\end{array}$ & $\begin{array}{c}0.621^{* * *} \\
(0.10)\end{array}$ & $\begin{array}{c}0.509 * * * \\
(0.11)\end{array}$ \\
\hline Female & & & $\begin{array}{r}-0.023 \\
(0.09)\end{array}$ & $\begin{array}{l}0.026 \\
(0.09)\end{array}$ \\
\hline Freshman & & & $\begin{array}{c}0.273^{* *} \\
(0.09)\end{array}$ & $\begin{array}{c}0.238^{*} \\
(0.09)\end{array}$ \\
\hline Strengthening bus system & & & & $\begin{array}{c}1.179^{* * *} \\
(0.18)\end{array}$ \\
\hline Constant & $\begin{array}{c}-0.710^{* * *} \\
(0.06) \\
\end{array}$ & $\begin{array}{c}-0.826^{* * *} \\
(0.07) \\
\end{array}$ & $\begin{array}{c}-0.915^{* * *} \\
(0.09) \\
\end{array}$ & $\begin{array}{c}-0.959^{* * *} \\
(0.09) \\
\end{array}$ \\
\hline Pseudo $\mathrm{R}^{2}$ & 0.224 & 0.245 & 0.252 & 0.285 \\
\hline Log Likelihood & -668.1 & -623.5 & -589.1 & -539.1 \\
\hline Observations & 1242 & 1192 & 1137 & 1090 \\
\hline
\end{tabular}


Table 2.A.3: Culture ticket - Dataset I, coefficients for Table 2.10

\begin{tabular}{|c|c|c|c|c|}
\hline \multicolumn{5}{|c|}{ Dependent Variable: Supporting Culture Ticket } \\
\hline & (1) & (2) & (3) & (4) \\
\hline Rarely (1 or $2 /$ year) & $\begin{array}{c}0.982^{* * *} \\
(0.09)\end{array}$ & $\begin{array}{c}0.902^{* * *} \\
(0.10)\end{array}$ & $\begin{array}{c}0.961^{* * *} \\
(0.10)\end{array}$ & $\begin{array}{c}0.931^{* * *} \\
(0.12)\end{array}$ \\
\hline Sometimes ( 3 to $5 /$ year) & $\begin{array}{c}1.744^{* * *} \\
(0.15)\end{array}$ & $\begin{array}{c}1.643^{* * *} \\
(0.16)\end{array}$ & $\begin{array}{c}1.677^{* * *} \\
(0.17)\end{array}$ & $\begin{array}{c}1.664^{* * *} \\
(0.22)\end{array}$ \\
\hline Often (6 to $10 /$ year) & $\begin{array}{c}2.164 * * * \\
(0.33)\end{array}$ & $\begin{array}{c}2.083^{* * *} \\
(0.34)\end{array}$ & $\begin{array}{c}2.138^{* * *} \\
(0.35)\end{array}$ & $\begin{array}{c}1.901^{* * *} \\
(0.44)\end{array}$ \\
\hline Very often $(>10 /$ year $)$ & $\begin{array}{c}1.915^{* * *} \\
(0.30)\end{array}$ & $\begin{array}{c}1.717^{* * *} \\
(0.31)\end{array}$ & $\begin{array}{c}1.855^{* * * *} \\
(0.36)\end{array}$ & $\begin{array}{c}1.523^{* *} \\
(0.48)\end{array}$ \\
\hline Savings of others & & $\begin{array}{c}0.727^{* * *} \\
(0.11)\end{array}$ & $\begin{array}{c}0.726^{* * * *} \\
(0.11)\end{array}$ & $\begin{array}{c}0.405^{* *} \\
(0.15)\end{array}$ \\
\hline Female & & & $\begin{array}{c}0.220^{*} \\
(0.09)\end{array}$ & $\begin{array}{l}0.085 \\
(0.10)\end{array}$ \\
\hline Freshman & & & $\begin{array}{c}0.327^{* *} \\
(0.10)\end{array}$ & $\begin{array}{c}0.338^{* *} \\
(0.12)\end{array}$ \\
\hline Others should go & & & & $\begin{array}{c}1.293^{* * *} \\
(0.19)\end{array}$ \\
\hline Strengthening local culture & & & & $\begin{array}{c}1.344^{* * *} \\
(0.13)\end{array}$ \\
\hline Constant & $\begin{array}{c}-0.463^{* * *} \\
(0.05) \\
\end{array}$ & $\begin{array}{c}-0.536^{* * *} \\
(0.06) \\
\end{array}$ & $\begin{array}{c}-0.767^{* * * *} \\
(0.08) \\
\end{array}$ & $\begin{array}{c}-1.141^{* * *} \\
(0.10) \\
\end{array}$ \\
\hline Pseudo $\mathrm{R}^{2}$ & 0.192 & 0.215 & 0.215 & 0.440 \\
\hline Log Likelihood & -664.7 & -599.2 & -571.1 & -378.7 \\
\hline Observations & 1189 & 1110 & 1055 & 988 \\
\hline
\end{tabular}


Table 2.A.4: Bahn ticket - Dataset II, coefficients for Table 2.11

\begin{tabular}{|c|c|c|c|c|c|}
\hline \multicolumn{6}{|c|}{ Dependent Variable: Supporting Bahn Ticket } \\
\hline & (1) & $(2)$ & $(3)$ & (4) & $(5)$ \\
\hline \multirow[t]{2}{*}{ Log savings } & $0.258^{* * *}$ & $0.215^{* * *}$ & $0.230^{* * *}$ & $0.268^{* * *}$ & $0.271^{* * *}$ \\
\hline & $(0.02)$ & $(0.02)$ & $(0.02)$ & $(0.03)$ & $(0.03)$ \\
\hline \multirow[t]{2}{*}{ Leisure/work } & & 0.244 & 0.104 & 0.143 & 0.158 \\
\hline & & $(0.24)$ & $(0.26)$ & $(0.28)$ & $(0.28)$ \\
\hline \multirow[t]{2}{*}{ Visiting others } & & $1.000 * * *$ & $1.044 * * *$ & $0.951 * * *$ & $0.975 * * *$ \\
\hline & & $(0.11)$ & $(0.12)$ & $(0.13)$ & $(0.13)$ \\
\hline \multirow[t]{2}{*}{ Female } & & & 0.175 & 0.149 & 0.145 \\
\hline & & & $(0.12)$ & $(0.13)$ & $(0.13)$ \\
\hline \multirow[t]{2}{*}{ Freshman } & & & $0.326^{*}$ & $0.437^{*}$ & $0.465^{*}$ \\
\hline & & & $(0.16)$ & $(0.18)$ & $(0.19)$ \\
\hline \multirow[t]{2}{*}{ Altruist $(-)$} & & & & $-0.538^{* *}$ & $-0.526^{* *}$ \\
\hline & & & & $(0.18)$ & $(0.18)$ \\
\hline \multirow{2}{*}{ Altruist $(+)$} & & & & $1.023 * * *$ & $1.031 * * *$ \\
\hline & & & & $(0.17)$ & $(0.17)$ \\
\hline \multirow[t]{2}{*}{ Protest } & & & & & -0.282 \\
\hline & & & & & $(0.15)$ \\
\hline \multirow[t]{2}{*}{ Constant } & $-0.220 * * *$ & $-0.728 * * *$ & $-0.872 * * *$ & $-1.112^{* * *}$ & $-1.062 * * *$ \\
\hline & $(0.07)$ & $(0.09)$ & $(0.12)$ & $(0.15)$ & $(0.15)$ \\
\hline Pseudo $\mathrm{R}^{2}$ & 0.225 & 0.306 & 0.333 & 0.434 & 0.438 \\
\hline Log Likelihood & -395.7 & -351.7 & -307.4 & -236.0 & -234.2 \\
\hline Observations & 818 & 810 & 741 & 669 & 669 \\
\hline
\end{tabular}

Table 2.A.5: Taking part - Dataset II, coefficients for Table 2.12

\begin{tabular}{|c|c|c|c|c|c|c|}
\hline \multicolumn{7}{|c|}{ Dependent Variable: Taking Part in Referendum } \\
\hline & (1) & $(2)$ & $(3)$ & (4) & $(5)$ & $(6)$ \\
\hline & all & all & all & all & savings $\geq$ price & savings $<$ price \\
\hline \multirow[t]{2}{*}{ Log stakes } & $0.206^{* * *}$ & $0.205^{* * *}$ & $0.223^{* * *}$ & $0.228^{* * *}$ & $0.171^{* *}$ & 0.109 \\
\hline & $(0.04)$ & $(0.04)$ & $(0.04)$ & $(0.04)$ & $(0.06)$ & $(0.10)$ \\
\hline \multirow[t]{2}{*}{ Leisure/work } & & -0.047 & -0.021 & -0.025 & -0.050 & -0.007 \\
\hline & & $(0.16)$ & $(0.18)$ & $(0.18)$ & $(0.31)$ & $(0.22)$ \\
\hline \multirow[t]{2}{*}{ Visiting others } & & 0.120 & 0.101 & 0.095 & 0.140 & -0.024 \\
\hline & & $(0.08)$ & $(0.09)$ & $(0.09)$ & $(0.18)$ & $(0.10)$ \\
\hline \multirow[t]{2}{*}{ Female } & & & $-0.300 * * *$ & $-0.298 * * *$ & $-0.396^{* *}$ & $-0.225^{*}$ \\
\hline & & & $(0.08)$ & $(0.08)$ & $(0.14)$ & $(0.10)$ \\
\hline \multirow[t]{2}{*}{ Freshman } & & & $0.489 * * *$ & $0.481 * * *$ & 0.350 & $0.581 * * *$ \\
\hline & & & $(0.13)$ & $(0.13)$ & $(0.20)$ & $(0.17)$ \\
\hline \multirow[t]{2}{*}{ Protest } & & & & $0.279^{* *}$ & 0.252 & $0.265^{*}$ \\
\hline & & & & $(0.10)$ & $(0.17)$ & $(0.13)$ \\
\hline \multirow[t]{2}{*}{ Constant } & $-0.479 * *$ & $-0.542^{* *}$ & $-0.502^{*}$ & $-0.578^{* *}$ & -0.096 & -0.128 \\
\hline & $(0.18)$ & $(0.18)$ & $(0.20)$ & $(0.20)$ & $(0.34)$ & $(0.47)$ \\
\hline Pseudo $\mathrm{R}^{2}$ & 0.023 & 0.026 & 0.050 & 0.056 & 0.046 & 0.027 \\
\hline Log Likelihood & -713.1 & -700.8 & -625.6 & -622.0 & -213.2 & -403.5 \\
\hline Observations & 1189 & 1174 & 1075 & 1075 & 449 & 626 \\
\hline
\end{tabular}

Probit estimation, coefficients, standard errors in parentheses. Regression (5): only students whose savings cover ticket costs (winners), regression (6): only students whose savings do not cover ticket costs. $* p<0.05, * *<0.01, * * * p<0.001$ 
Table 2.A.6: Taking part, reduced sample - Dataset II, coefficients for Table 2.13

\begin{tabular}{|c|c|c|c|c|c|c|}
\hline \multicolumn{7}{|c|}{ Dependent Variable: Taking Part in Referendum } \\
\hline & (1) & $(2)$ & $(3)$ & $(4)$ & $(5)$ & $(6)$ \\
\hline Bottom & $95 \%$ & $90 \%$ & $85 \%$ & $80 \%$ & $75 \%$ & $70 \%$ \\
\hline \multirow[t]{2}{*}{ Log stakes } & $0.235^{* * *}$ & $0.204^{* * *}$ & $0.188^{* * *}$ & $0.140^{*}$ & 0.078 & 0.030 \\
\hline & $(0.05)$ & $(0.05)$ & $(0.06)$ & $(0.06)$ & $(0.07)$ & $(0.08)$ \\
\hline \multirow[t]{2}{*}{ Leisure/work } & -0.018 & -0.032 & -0.059 & -0.030 & -0.014 & -0.074 \\
\hline & $(0.18)$ & $(0.19)$ & $(0.19)$ & $(0.19)$ & $(0.20)$ & $(0.21)$ \\
\hline \multirow[t]{2}{*}{ Visiting others } & 0.110 & 0.110 & 0.121 & 0.101 & 0.062 & 0.018 \\
\hline & $(0.09)$ & $(0.09)$ & $(0.09)$ & $(0.09)$ & $(0.09)$ & $(0.10)$ \\
\hline \multirow[t]{2}{*}{ Female } & $-0.288^{* * *}$ & $-0.282^{* *}$ & $-0.270^{* *}$ & $-0.244^{* *}$ & $-0.252^{* *}$ & $-0.209^{*}$ \\
\hline & $(0.09)$ & $(0.09)$ & $(0.09)$ & $(0.09)$ & $(0.09)$ & $(0.09)$ \\
\hline \multirow[t]{2}{*}{ Freshman } & $0.497 * * *$ & $0.494^{* * *}$ & $0.463^{* * *}$ & $0.496 * * *$ & $0.484^{* *}$ & $0.469 * *$ \\
\hline & $(0.14)$ & $(0.14)$ & $(0.14)$ & $(0.15)$ & $(0.15)$ & $(0.16)$ \\
\hline \multirow[t]{2}{*}{ Protest } & $0.304^{* *}$ & $0.305^{* *}$ & $0.288^{* *}$ & $0.285^{*}$ & $0.313^{* *}$ & $0.300^{*}$ \\
\hline & $(0.11)$ & $(0.11)$ & $(0.11)$ & $(0.11)$ & $(0.11)$ & $(0.12)$ \\
\hline \multirow[t]{2}{*}{ Constant } & $-0.630 * *$ & $-0.501 *$ & -0.438 & -0.248 & 0.024 & 0.219 \\
\hline & $(0.22)$ & $(0.24)$ & $(0.26)$ & $(0.29)$ & $(0.32)$ & $(0.35)$ \\
\hline Pseudo $\mathrm{R}^{2}$ & 0.052 & 0.044 & 0.036 & 0.030 & 0.027 & 0.022 \\
\hline Log Likelihood & -594.8 & -580.7 & -558.1 & -535.0 & -509.4 & -486.7 \\
\hline Observations & 1011 & 967 & 912 & 858 & 806 & 761 \\
\hline
\end{tabular}

Probit estimation, coefficients, standard errors in parentheses. Columns show percentiles with respect to stakes, e.g. column (1) contains those observations who belong to the bottom $95 \%$ with respect to stakes. ${ }^{*} p<0.05,{ }^{* *} p<0.01,{ }^{* * *} p<0.001$. 


\section{Appendix 2.A.IV: Political parties and fields of study}

Table 2.A.7: General political preferences - Dataset I, coefficients

\begin{tabular}{|c|c|c|c|}
\hline \multicolumn{4}{|c|}{ Dependent Variable: Supporting Ticket } \\
\hline & $\begin{array}{c}(1) \\
\text { Train }\end{array}$ & $\begin{array}{l}\text { (2) } \\
\text { Bus }\end{array}$ & $\begin{array}{c}(3) \\
\text { Culture } \\
\end{array}$ \\
\hline Rarely $\diamond$ & $\begin{array}{c}1.034^{* * *} \\
(0.21)\end{array}$ & $\begin{array}{c}0.585^{* * *} \\
(0.11)\end{array}$ & $\begin{array}{c}0.893^{* * *} \\
(0.14)\end{array}$ \\
\hline Sometimes $\diamond$ & $\begin{array}{c}1.930^{* * *} \\
(0.22)\end{array}$ & $\begin{array}{c}1.083^{* * *} \\
(0.15)\end{array}$ & $\begin{array}{c}1.712^{* * *} \\
(0.25)\end{array}$ \\
\hline Often $\diamond$ & $\begin{array}{c}2.408^{* * *} \\
(0.26)\end{array}$ & $\begin{array}{c}1.626^{* * *} \\
(0.18)\end{array}$ & $\begin{array}{c}1.804^{* * * *} \\
(0.44)\end{array}$ \\
\hline Very often $\diamond$ & $\begin{array}{c}2.344^{* * *} \\
(0.25)\end{array}$ & $\begin{array}{c}2.104^{* * *} \\
(0.20)\end{array}$ & $\begin{array}{c}1.981^{* * * *} \\
(0.60)\end{array}$ \\
\hline Savings of others & $\begin{array}{c}0.690^{* * * *} \\
(0.15)\end{array}$ & $\begin{array}{c}0.538^{* * * *} \\
(0.12)\end{array}$ & $\begin{array}{c}0.366^{*} \\
(0.16)\end{array}$ \\
\hline Female & $\begin{array}{l}0.332^{*} \\
(0.13)\end{array}$ & $\begin{array}{l}0.040 \\
(0.10)\end{array}$ & $\begin{array}{c}-0.049 \\
(0.12)\end{array}$ \\
\hline Freshman & $\begin{array}{l}-0.176 \\
(0.14)\end{array}$ & $\begin{array}{c}0.272^{* *} \\
(0.10)\end{array}$ & $\begin{array}{l}0.346^{*} \\
(0.14)\end{array}$ \\
\hline Environment & $\begin{array}{c}0.663^{* * *} \\
(0.19)\end{array}$ & & \\
\hline Strengthening bus system & & $\begin{array}{c}1.225^{* * *} \\
(0.20)\end{array}$ & \\
\hline Others should go & & & $\begin{array}{c}1.267^{* * *} \\
(0.20)\end{array}$ \\
\hline Strengthening local culture & & & $\begin{array}{c}1.287^{* * *} \\
(0.15)\end{array}$ \\
\hline Social Democrats & $\begin{array}{l}-0.104 \\
(0.16)\end{array}$ & $\begin{array}{l}0.017 \\
(0.12)\end{array}$ & $\begin{array}{c}0.628^{* * * *} \\
(0.15)\end{array}$ \\
\hline Liberal Democrats & $\begin{array}{c}-0.562 \\
(0.29)\end{array}$ & $\begin{array}{l}-0.232 \\
(0.27)\end{array}$ & $\begin{array}{l}0.171 \\
(0.27)\end{array}$ \\
\hline Green & $\begin{array}{l}-0.104 \\
(0.17)\end{array}$ & $\begin{array}{l}-0.109 \\
(0.13)\end{array}$ & $\begin{array}{c}0.611^{* * *} \\
(0.16)\end{array}$ \\
\hline Left & $\begin{array}{l}-0.065 \\
(0.34)\end{array}$ & $\begin{array}{l}0.037 \\
(0.25)\end{array}$ & $\begin{array}{c}0.970^{* *} \\
(0.34)\end{array}$ \\
\hline Other parties & $\begin{array}{c}0.153 \\
(0.31)\end{array}$ & $\begin{array}{l}-0.076 \\
(0.23)\end{array}$ & $\begin{array}{l}0.260 \\
(0.28)\end{array}$ \\
\hline Constant & $\begin{array}{c}-0.884^{* * *} * \\
(0.23) \\
\end{array}$ & $\begin{array}{c}-0.935^{* * *} \\
(0.11) \\
\end{array}$ & $\begin{array}{c}-1.486^{* * *} \\
(0.14)\end{array}$ \\
\hline Pseudo $\mathrm{R}^{2}$ & 0.371 & 0.281 & 0.466 \\
\hline Log Likelihood & -246.5 & -477.1 & -312.7 \\
\hline Observations & 1000 & 958 & 858 \\
\hline
\end{tabular}

Probit estimation, coefficients, standard errors in parentheses.

$\diamond$ Definitions of the intensity of use differ: rarely, sometimes, often,

very often corresponds to $\leq 5 /$ year, monthly, weekly, $\geq 2 /$ week for the train ticket, 1 or 2 /semester, monthly, weekly, $\geq 2$ /week during lecture period for the bus ticket and 1 or 2 /year, 3 to 5 /year, 6 to $10 /$ year, $>10 /$ year for the culture ticket. The base category for the party preferences is Christian Democrats. ${ }^{*} p<0.05,{ }^{* *} p<0.01,{ }^{* * *} p<0.001$ 
Table 2.A.8: Fields of study - Dataset I, coefficients

\begin{tabular}{|c|c|c|c|}
\hline \multicolumn{4}{|c|}{ Dependent Variable: Supporting Ticket } \\
\hline & $\begin{array}{c}(1) \\
\text { Train }\end{array}$ & $\begin{array}{l}(2) \\
\text { Bus }\end{array}$ & $\begin{array}{c}(3) \\
\text { Culture }\end{array}$ \\
\hline Rarely $^{\triangleright}$ & $\begin{array}{c}1.083^{* * *} \\
(0.20)\end{array}$ & $\begin{array}{c}0.640^{* * *} \\
(0.11)\end{array}$ & $\begin{array}{c}0.859^{* * *} \\
(0.13)\end{array}$ \\
\hline Sometimes $\diamond$ & $\begin{array}{c}2.010^{* * *} \\
(0.21)\end{array}$ & $\begin{array}{c}1.174^{* * *} \\
(0.14)\end{array}$ & $\begin{array}{c}1.642^{* * *} \\
(0.23)\end{array}$ \\
\hline Often $\diamond$ & $\begin{array}{l}2.402^{* * *} \\
(0.24)\end{array}$ & $\begin{array}{c}1.644^{* * *} \\
(0.17)\end{array}$ & $\begin{array}{c}1.755^{* * *} \\
(0.45)\end{array}$ \\
\hline Very often $\diamond$ & $\begin{array}{c}2.438^{* * *} \\
(0.25)\end{array}$ & $\begin{array}{c}2.237^{* * *} \\
(0.19)\end{array}$ & $\begin{array}{c}1.430^{* *} \\
(0.51)\end{array}$ \\
\hline Savings of others & $0.632^{* * *}$ & $0.500^{* * *}$ & $0.398^{* *}$ \\
\hline Female & $\begin{array}{c}0.186 \\
(0.13)\end{array}$ & $\begin{array}{c}0.025 \\
(0.09)\end{array}$ & $\begin{array}{l}0.015 \\
(0.11)\end{array}$ \\
\hline Freshman & $\begin{array}{l}-0.117 \\
(0.13)\end{array}$ & $\begin{array}{l}0.242^{*} \\
(0.10)\end{array}$ & $\begin{array}{l}0.319^{*} \\
(0.13)\end{array}$ \\
\hline Environment & $\begin{array}{c}0.707^{* * *} \\
(0.18)\end{array}$ & & \\
\hline Strengthening bus system & & $\begin{array}{c}1.177^{* * *} \\
(0.18)\end{array}$ & \\
\hline Others should go & & & $\begin{array}{c}1.333^{* * *} * \\
(0.19)\end{array}$ \\
\hline Strengthening local culture & & & $\begin{array}{c}1.317^{* * *} \\
(0.14)\end{array}$ \\
\hline Social sciences & $\begin{array}{c}0.149 \\
(0.17)\end{array}$ & $\begin{array}{c}0.086 \\
(0.12)\end{array}$ & $\begin{array}{c}0.560^{* * *} \\
(0.14)\end{array}$ \\
\hline Forestry/Agriculture & $\begin{array}{c}-0.623^{* *} \\
(0.22)\end{array}$ & $\begin{array}{c}0.262 \\
(0.19)\end{array}$ & $\begin{array}{r}-0.147 \\
(0.24)\end{array}$ \\
\hline Humanities & $\begin{array}{c}0.210 \\
(0.16)\end{array}$ & $\begin{array}{l}0.040 \\
(0.11)\end{array}$ & $\begin{array}{l}0.343^{*} \\
(0.14)\end{array}$ \\
\hline Geology/Geography & $\begin{array}{l}0.016 \\
(0.41)\end{array}$ & $\begin{array}{l}0.082 \\
(0.26)\end{array}$ & $\begin{array}{l}0.545 \\
(0.32)\end{array}$ \\
\hline Law & $\begin{array}{l}-0.046 \\
(0.20)\end{array}$ & $\begin{array}{l}0.318^{*} \\
(0.15)\end{array}$ & $\begin{array}{l}0.089 \\
(0.17)\end{array}$ \\
\hline Natural sciences & $\begin{array}{l}-0.191 \\
(0.21)\end{array}$ & $\begin{array}{l}-0.026 \\
(0.17)\end{array}$ & $\begin{array}{l}0.440^{*} \\
(0.20)\end{array}$ \\
\hline Other fields & $\begin{array}{l}0.397 \\
(0.47)\end{array}$ & $\begin{array}{l}-0.146 \\
(0.25)\end{array}$ & $\begin{array}{l}0.240 \\
(0.33)\end{array}$ \\
\hline Constant & $\begin{array}{c}-0.949 * * * \\
(0.21)\end{array}$ & $\begin{array}{c}-1.039^{* * *} \\
(0.10)\end{array}$ & $\begin{array}{c}-1.336 * * * \\
(0.12)\end{array}$ \\
\hline Pseudo $\mathrm{R}^{2}$ & 0.381 & 0.290 & 0.460 \\
\hline Log Likelihood & -270.4 & -534.9 & -364.3 \\
\hline Observations & 1143 & 1088 & 986 \\
\hline
\end{tabular}

Probit estimation, coefficients, standard errors in parentheses.

$\diamond$ Definitions of the intensity of use differ: rarely, sometimes, often,

very often corresponds to $\leq 5 /$ year, monthly, weekly, $\geq 2$ /week

for the train ticket, 1 or $2 /$ semester, monthly, weekly, $\geq 2$ /week

during lecture period for the bus ticket and 1 or $2 /$ year, 3 to $5 /$ year, 6 to $10 /$ year, $>10$ year for the culture ticket. The base categories for the fields of study is economic sciences. ${ }^{*} p<0.05,{ }^{* *} p<0.01,{ }^{* * *} p<0.001$ 
Table 2.A.9: Political preferences and fields of study - Dataset I, coefficients

\begin{tabular}{|c|c|c|c|}
\hline \multicolumn{4}{|c|}{ Dependent Variable: Supporting Ticket } \\
\hline & $\begin{array}{c}(1) \\
\text { Train }\end{array}$ & $\begin{array}{l}(2) \\
\text { Bus }\end{array}$ & $\begin{array}{c}(3) \\
\text { Culture }\end{array}$ \\
\hline Rarely $\diamond$ & $\begin{array}{c}1.060^{* * *} \\
(0.22)\end{array}$ & $\begin{array}{l}0.583^{* * *} \\
(0.12)\end{array}$ & $\begin{array}{c}0.853^{* * *} \\
(0.14)\end{array}$ \\
\hline Sometimes $\diamond$ & $\begin{array}{c}1.963^{* * *} \\
(0.22)\end{array}$ & $\begin{array}{c}1.081^{* * *} \\
(0.15)\end{array}$ & $\begin{array}{c}1.728^{* * * *} \\
(0.25)\end{array}$ \\
\hline Often $\diamond$ & $\begin{array}{c}2.475^{* * *} \\
(0.26)\end{array}$ & $\begin{array}{c}1.619^{* * *} \\
(0.18)\end{array}$ & $\begin{array}{c}1.762^{* * * *} \\
(0.45)\end{array}$ \\
\hline Very often $\diamond$ & $\begin{array}{c}2.331^{* * *} \\
(0.26)\end{array}$ & $\begin{array}{l}2.112^{* * *} \\
(0.20)\end{array}$ & $\begin{array}{c}1.971 * * \\
(0.63)\end{array}$ \\
\hline Savings of others & $\begin{array}{c}0.718^{* * *} \\
(0.16)\end{array}$ & $\begin{array}{c}0.529^{* * *} \\
(0.12)\end{array}$ & $\begin{array}{l}0.361^{*} \\
(0.16)\end{array}$ \\
\hline Female & $\begin{array}{l}0.261 \\
(0.14)\end{array}$ & $\begin{array}{c}0.035 \\
(0.10)\end{array}$ & $\begin{array}{l}-0.062 \\
(0.12)\end{array}$ \\
\hline Freshman & $\begin{array}{l}-0.178 \\
(0.14)\end{array}$ & $\begin{array}{c}0.275^{* *} \\
(0.10)\end{array}$ & $\begin{array}{l}0.348^{*} \\
(0.14)\end{array}$ \\
\hline Environment & $\begin{array}{c}0.644^{* * *} \\
(0.19)\end{array}$ & & \\
\hline Strengthening bus system & & $\begin{array}{c}1.225^{* * *} \\
(0.20)\end{array}$ & \\
\hline Others should go & & & $\begin{array}{c}1.316^{* * *} \\
(0.20)\end{array}$ \\
\hline Strengthening local culture & & & $\begin{array}{c}1.294^{* * *} \\
(0.15)\end{array}$ \\
\hline Social sciences & $\begin{array}{l}0.172 \\
(0.18)\end{array}$ & $\begin{array}{l}0.127 \\
(0.12)\end{array}$ & $\begin{array}{c}0.446^{* *} \\
(0.15)\end{array}$ \\
\hline Forestry/Agriculture & $\begin{array}{c}-0.661^{* *} \\
(0.24)\end{array}$ & $\begin{array}{l}0.165 \\
(0.21)\end{array}$ & $\begin{array}{l}-0.004 \\
(0.27)\end{array}$ \\
\hline Humanities & $\begin{array}{c}0.201 \\
(0.17)\end{array}$ & $\begin{array}{l}0.033 \\
(0.12)\end{array}$ & $\begin{array}{c}0.176 \\
(0.16)\end{array}$ \\
\hline Geology/Geography & $\begin{array}{c}0.013 \\
(0.42)\end{array}$ & $\begin{array}{l}0.267 \\
(0.28)\end{array}$ & $\begin{array}{c}0.608 \\
(0.36)\end{array}$ \\
\hline Law & $\begin{array}{l}-0.097 \\
(0.21)\end{array}$ & $\begin{array}{l}0.414^{*} \\
(0.16)\end{array}$ & $\begin{array}{c}0.073 \\
(0.18)\end{array}$ \\
\hline Natural sciences & $\begin{array}{l}-0.367 \\
(0.23)\end{array}$ & $\begin{array}{l}0.059 \\
(0.18)\end{array}$ & $\begin{array}{c}0.379 \\
(0.22)\end{array}$ \\
\hline Other fields & $\begin{array}{l}0.328 \\
(0.52)\end{array}$ & $\begin{array}{l}-0.065 \\
(0.28)\end{array}$ & $\begin{array}{l}0.231 \\
(0.38)\end{array}$ \\
\hline Social Democrats & $\begin{array}{l}-0.231 \\
(0.17)\end{array}$ & $\begin{array}{l}0.046 \\
(0.13)\end{array}$ & $\begin{array}{c}0.541 * * * \\
(0.16)\end{array}$ \\
\hline Liberal Democrats & $\begin{array}{c}-0.674^{*} \\
(0.29)\end{array}$ & $\begin{array}{l}-0.218 \\
(0.27)\end{array}$ & $\begin{array}{c}0.178 \\
(0.27)\end{array}$ \\
\hline Green & $\begin{array}{l}-0.221 \\
(0.19)\end{array}$ & $\begin{array}{l}-0.081 \\
(0.13)\end{array}$ & $\begin{array}{c}0.466^{* *} \\
(0.17)\end{array}$ \\
\hline Left & $\begin{array}{l}-0.217 \\
(0.35)\end{array}$ & $\begin{array}{c}0.058 \\
(0.26)\end{array}$ & $\begin{array}{c}0.930^{* *} \\
(0.36)\end{array}$ \\
\hline Other parties & $\begin{array}{l}0.106 \\
(0.33)\end{array}$ & $\begin{array}{l}-0.037 \\
(0.24)\end{array}$ & $\begin{array}{l}0.238 \\
(0.29)\end{array}$ \\
\hline Constant & $\begin{array}{c}-0.780^{* *} \\
(0.24)\end{array}$ & $\begin{array}{c}-1.057^{* * * *} \\
(0.13)\end{array}$ & $\begin{array}{c}-1.612^{* * *} \\
(0.16)\end{array}$ \\
\hline Pseudo $\mathrm{R}^{2}$ & 0.391 & 0.287 & 0.477 \\
\hline Log likelihood & -238.6 & -472.9 & -306.0 \\
\hline Observations & 999 & 957 & 857 \\
\hline
\end{tabular}

Probit estimation, coefficients, standard errors in parentheses.

$\diamond$ Definitions of the intensity of use differ: rarely, sometimes, often, very often corresponds to $\leq 5 /$ year, monthly, weekly, $\geq 2$ /week for the train ticket, 1 or $2 /$ semester, monthly, weekly, 2 /week during lecture period for the bus ticket and 1 or $2 /$ year, 3 to 5 /year, 6 to 10 /year, $>10$ year for the culture ticket. The base categories for the fields of study and party preferences are economic sciences and Christian Democrats, respectively. ${ }^{*} p<0.05,{ }^{* *} p<0.01,{ }^{* * *} p<0.001$ 
Table 2.A.10: General political preferences - Dataset II, coefficients

\begin{tabular}{|c|c|}
\hline Dependent Variable: & Supporting Bahn Ticket \\
\hline Log savings & $\frac{(1)}{0.291^{* * *}}$ \\
\hline & $(0.03)$ \\
\hline Leisure/work & $\begin{array}{r}0.340 \\
(0.34)\end{array}$ \\
\hline Visiting others & $\begin{array}{c}1.002^{* * *} \\
(0.16)\end{array}$ \\
\hline Female & $\begin{array}{l}0.151 \\
(0.16)\end{array}$ \\
\hline Freshman & $\begin{array}{c}0.613^{* *} \\
(0.22)\end{array}$ \\
\hline Altruist $(-)$ & $\begin{array}{c}-0.636^{* *} \\
(0.21)\end{array}$ \\
\hline Altruist $(+)$ & $\begin{array}{c}1.130^{* * *} \\
(0.19)\end{array}$ \\
\hline Protest & $\begin{array}{c}-0.376^{*} \\
(0.17)\end{array}$ \\
\hline Social Democrats & $\begin{array}{l}0.028 \\
(0.23)\end{array}$ \\
\hline Liberal Democrats & -0.273 \\
\hline Green & $\begin{array}{c}-0.207 \\
(0.21)\end{array}$ \\
\hline Left & $\begin{array}{l}0.084 \\
(0.49)\end{array}$ \\
\hline Other parties & $\begin{array}{l}0.273 \\
(0.36)\end{array}$ \\
\hline Constant & $\begin{array}{c}-1.004^{* * *} \\
(0.21)\end{array}$ \\
\hline Pseudo $\mathrm{R}^{2}$ & 0.456 \\
\hline Log likelihood & -178.8 \\
\hline Observations & 534 \\
\hline $\begin{array}{l}\text { Probit estimation, } \\
\text { rors in parentheses. } \\
\text { the party preferences } \\
{ }^{*} p<0.05,{ }^{* *} p<0.01,{ }^{* *}\end{array}$ & $\begin{array}{l}\text { coefficients, standard er- } \\
\text { The base category for } \\
\text { is Christian Democrats. } \\
* p<0.001 \text {. }\end{array}$ \\
\hline
\end{tabular}




\section{Chapter 3}

\section{Determinants of Students' Success at University*}

\section{$3.1 \quad$ Introduction}

The number of students in higher education worldwide is constantly increasing. Today's students are more heterogeneous than ever before and possess a wide and diverse range of characteristics and abilities. They often differ in educational background, social status, skills, and academic potential, among others. As the diversity of the student population increases, factors predicting students' academic performance become a matter of concern for institutions in the educational sector (see e.g. Burton and Dowling, 2005; Simpson, 2006). For instance, knowledge about factors affecting academic success is relevant for universities when selecting the most promising students. At an aggregate level, based on such knowledge, policy can decide to what extent investment in tertiary education should be directed towards those fields where large numbers of students can expect to succeed, or be concentrated in fields which rather cater to a minority of excellent students.

In this chapter we address this concern by focusing on the question of whether, and if so to what extent student characteristics can be used for predicting academic

*This chapter originates from joint work with Kamila Danilowicz-Gösele, Katharina Lerche (née Suntheim) and Robert Schwager (see Danilowicz-Gösele et al., 2014). 
success. We find a highly significant and positive effect of the high school leaving grade on academic performance. Additionally, we narrow our view towards differences between fields of study, grouped by faculties. We find that the importance of the high school leaving grade differs strongly between fields. In some faculties graduation is less difficult to achieve, but not necessarily associated with a good final grade. However, in other faculties, graduation seems to be less likely, but among those students who graduate, the final university grade is on average better and less differentiated. This points towards diverging teaching and examination cultures among faculties. Some of them specialize in preparing a positive selection of students to science or demanding employment, whereas others provide an education which is accessible for large numbers of high school graduates with average abilities.

The probability of academic success and the reasons for dropping out of university are subject of the continuously expanding research literature in many areas, notably economics of education, psychology and sociology. These studies provide a consistent picture of previous high school performance as the most prominent predictor of university success (Baron-Boldt, 1989; Betts and Morell, 1999; Cyrenne and Chan, 2012). Furthermore, various other personal characteristics are found to affect students' academic performance, for instance gender (McNabb et al., 2002), age (Hong, 1984) or socio-economic status (Arulampalam et al., 2005). Besides, the type of high school visited is shown to influence both the probability of entering a college (Altonji et al, 2005) as well as the probability of obtaining a good degree (Smith and Naylor, 2005). At university, also the chosen field of study might matter (Achen and Courant, 2009).

Although there is a vast amount of literature on factors predicting academic success, the analysis presented in this chapter differs from previous work in this area in a number of ways. Firstly, to the best of our knowledge, this is the first study that analyzes a comprehensive administrative data set of student population, that aims to be an encompassing analysis of students' characteristics as predictors for academic success at university in Germany. In contrast to much of the earlier work, we can track students' academic careers from the admission day onward. For instance, we observe changes in fields of study. Secondly, we analyze not only one but three dimensions of academic success: graduation from the university, graduation 
within a chosen field of study and final grade of the university degree. Thirdly, differentiating between faculties allows us to observe different examination cultures.

The remainder of this chapter is structured as follows: In Section 3.2 we present a brief overview of the related literature. In Section 3.3 we describe our dataset, explain the variables used, and lay out the empirical setup. We turn our attention to our empirical results in Section 3.4 and conclude with a discussion of the implications of these results in Section 3.5.

\subsection{Literature}

As the universities' selection process is often based on high school performance, almost all literature dealing with students' academic performance examines in the first place whether the high school Grade Point Average (GPA) is a valid predictor for university success. According to the meta-analysis of Robbins et al. (2004), the correlation between secondary school grades and university GPA is on average about 0.41 . Trapmann et al. (2007) find a mean corrected validity between 0.26 and 0.53 for high school grades predicting university success by using a meta-analysis approach including studies from Austria, Czech Republic, Germany, Great Britain and Norway. In this sample, the German high school GPA has the highest validity.

However, the predictive effectiveness of secondary school grades on academic performance seems to be different for diverse groups. For instance, Dobson and Skuja (2005) show that high university entrance scores are indeed a good predictor, but not for every field of study. They find a strong correlation between the university entrance scores and students' academic performance in agriculture, engineering and science, and almost no correlation in education and health studies. This corresponds to the results of Trapmann et al. (2007) who find a high predictive power for engineering and natural sciences and a comparatively low validity for psychology.

There is also a large number of contributions showing that students with the same entry grades are often found to perform differently in tertiary education, which suggests the importance of other factors when predicting university success. Based on an analysis of about 300 students in a regional equity and access program of Monash University, Australia, Levy and Murray (2005) report that an appropriate 
coaching program can reduce the impact of discrepancy in university entrance scores. Consequently, the entrance scores themselves may not be able to capture all relevant student characteristics.

In a study by Grebennikov and Skaines (2009) at the University of Western Sydney, data relating to about 9,000 students was analyzed in order to determine a set of variables predicting students' academic performance and retention. They find that the odds of dropping out without applying to other educational institutions are significantly higher for part-time and mature students, who tend to have less time for studying and face stricter financial constraints. Furthermore, the probability of early withdrawal from university is particularly high for students from an Englishspeaking background and with a low grade point average.

An analysis of academic, psychological, cognitive, and demographic predictors for academic performance can be found in McKenzie and Schweitzer (2001). For this purpose, they examine a group of about 200 first year students and find significant coefficients for the university entry score (accounting for $39 \%$ of the variance in GPA), student institution integration (accounting for $3 \%$ of the variance in GPA) and self-efficacy (accounting for $8 \%$ of the variance in the GPA). When both the measure of integration and the measure of self-efficacy are included in the model, the prediction of GPA at university is improved by $12 \%$.

Looking at a data set of the population of newly enrolled students at the University of Brussels, Arias Ortiz and Dehon (2008) examine the probability of succeeding the first year at university by accounting for individual characteristics, prior schooling and socio-economic background. According to their results, socio-economic background, especially the mother's level of education and the father's occupational activity, matters for students' academic success. In addition, they observe differences in academic performance between students coming from different high school programs.

Other factors mentioned in the literature that may help identify students at risk of failing include: standardized pre-university tests (Cohn et al., 2004), study skills (Robbins et al., 2004), the ability to adapt to the university environment (McInnis et al., 2000; Peat et al., 2001) or first year experience at the university (Krause et al., 2005). Further studies emphasize the importance of psychosocial variables such 
as goal and institutional commitment (Tinto, 1975), emotional intelligence (Parker et al., 2004), relationship with the faculty (Girves and Wemmerus, 1988) and social support (Gerdes and Mallinckrodt, 1994).

Altogether it appears to be generally accepted that high school performance is the best predictor for university success. We confirm this result using a new and comprehensive dataset from a German university. Contrary to the mixed results about the link between high school GPA and success in specific fields, we find that such a link is present in all faculties, albeit in different forms. Specifically, by distinguishing between several measures of success, we are able to describe in detail how this relationship varies across fields. Finally, again contrasting with some of the results cited, our data does not support the view that social origin or income have strong additional impact on university success once high school grades are taken into account.

\subsection{Data and approach}

In our analysis we use an extensive administrative dataset from Goettingen University, Germany, which encompasses detailed, anonymized information on more than 12,000 students. One part of the data is collected when students enroll at university and contains information about the student's high school leaving certificate, his or her parental address, gender and type of health insurance. The other part includes information about the student's university career, such as the field of study, the reason for leaving university, whether he or she obtained a degree and if so, which one.

In addition, we use data on the purchasing power of the German zip-code areas which is provided by $G f K$, a market research firm. ${ }^{13}$ The index is based on data provided by the German tax offices as well as other relevant statistics, for instance regarding pensions and unemployment benefits.

Detailed information on data filtering and processing can be found in Appendix 3.A.I.

\footnotetext{
$13 G f K$ is one of the biggest companies worldwide in the field of market research and collects information on people's lifestyle and consumption behavior.
} 


\subsubsection{Variable description and institutional background}

We use the following three measures of university success: the probability of finishing studies with a degree, the probability of finishing a chosen field of study with a degree and the grade of the final university degree. For the first two measures, it is necessary to distinguish between students who drop out and those who change institution. For this reason, we exclude students who mention that they leave Goettingen University in order to continue studying at another university from the sample.

As one is generally considered to be a successful student if one holds some degree after finishing university, we first examine a binary variable which describes whether the student graduates at all from university. The variable is equal to one for all students who finish their studies with any kind of degree at Goettingen University, and zero otherwise.

However, since in Germany students have to decide on their field of study as soon as they register for university, it is not uncommon that more than one subject is chosen or that the major is changed within the first few years. Therefore, we narrow down the definition of university success by using an additional outcome variable, labeled 'graduation within faculty', measuring success in each program the student enrolled in. This implies that when a student changes his or her field of study or enrolls in more than one degree program, several observations are generated. Thereby, success or failure are registered individually for every observation dependent on whether the student obtained a degree in this specific field of study or not. For example, for a student who changed his or her subject of study once during his or her university career and completed only the second study subject, the dataset will contain two observations. For the first observation, the variable describing success equals zero, and for the second, it is one. However, as study programs within the same faculty are typically quite similar with respect to their content or required abilities, a change of subject is only seen as a failure if it also implies a change of the faculty.

The third outcome variable is the grade of the university degree. As some students are enrolled in more than one study program or complete two consecutive degree programs, we create individual observations for every final university degree 
obtained. Furthermore, we transform grades into the U.S. grading scale in order to make results internationally comparable and easier to interpret. In Germany, the grading schedule traditionally ranges from 1.0 to 5.0, with 1.0 being the best grade to achieve and 4.0 the worst grade that is still a pass. This implies that the better the performance, the lower the grade. The outcome variable university GPA, which we use in our analysis, is a transformation of the actual grade achieved. It ranges from 1.0 to 4.0 with 4.0 being the best grade to obtain and 1.0 the worst that is still a pass. ${ }^{14}$

The central exogenous variable used in the analysis is the high school GPA, a transformation of the grade of the high school leaving certificate. Similar to the grade of the university degree, it is converted to the U.S. grading scale with 4.0 being the best and 1.0 the worst passing grade.

The students' socio-economic background is captured by two variables: the type of health insurance and the purchasing power of the parents' zip-code area.

Due to a particular institutional feature of the German health insurance system, the type of health insurance can be used as a proxy for the students' educational and socio-economic background. In order to choose a private instead of the generally compulsory public health insurance, one has to earn more than a certain amount of income (2013 : 52,200 euros gross income per year), be self-employed or work as civil servant. As most students are insured through their parents, the type of health insurance a student holds contains information about whether his or her parents satisfy at least one of the above criteria. Specifically, a large group of civil servants are teachers, and many self-employed and high earners hold a university degree. Overall, in 2008, 56.7 percent of the people being privately insured held a degree enabling registration at a university or a university of applied science, 38.0 percent had completed university or university of applied science with a degree or a $\mathrm{Ph} . \mathrm{D}$ (Finkenstädt and Keßler, 2012). Within the total German population, these shares were much lower, amounting to 24.4 and 13.0 percent respectively (Statistisches Bundesamt, 2009).

The second socio-economic variable we use is an index of the purchasing power

\footnotetext{
${ }^{14}$ We transformed the grades into the U.S. grading scale by subtracting the final university grade from five. For legal studies the special grade "vollbefriedigend" is treated as a 2.5.
} 
within the zip-code area of the student's home address evaluated in the year 2007. The index, provided by $G f K$, is measured relative to the German average, and normalized to 100. For example, an index value of 110 means that the purchasing power of this area is $10 \%$ higher than the German average. Since German zip-code areas are fairly small, with the biggest cities like Hamburg or Berlin encompassing up to about 190 different zip-codes, and assuming a certain degree of residential sorting according to income, we are confident that this local measure approximates the students' economic background reasonably well.

As additional covariates we include indicator variables for male students, the sixteen German states and the university's thirteen faculties.

To get a more diversified picture of the determinants of university success, we also divide the data into sub-samples by faculty. At Goettingen University the various fields of study are assigned to thirteen faculties: theology, law, medicine, humanities, mathematics, physics, chemistry, geology/geography, biology, forestry, agriculture, economic sciences, and social sciences. A detailed analysis of individual faculties seems worthwhile since they may differ with regard to scientific approach, organizational structure and general conditions of studying.

\subsubsection{Summary statistics}

Summary statistics are presented in Table 3.1. The final dataset contains 12,315 students out of which $48 \%$ obtained a degree at Goettingen University. The remaining $52 \%$ left Goettingen University without completing a degree. Taking into account that students might be enrolled in more than one degree program or change fields of study during their university career increases the number of observations to 16,931 . For $49 \%$ of these observations the respective field of study is completed with a degree.

When taking a look at those students who graduated, we see that a final grade is registered for 8,204 observations. This implies that around one third of the students who finished their studies obtained more than one university degree. The reason for this could be the introduction of the consecutive study programs which by definition leads to more than one degree for many students. 
Table 3.1: Summary statistics

\begin{tabular}{lccccc}
\hline \hline Variable & $\mathrm{N}$ & Mean & Std. Dev. & Min & Max \\
\hline High school GPA & 12315 & 2.50 & 0.63 & 1.10 & 4.00 \\
Graduation (university) & 12315 & 0.48 & 0.50 & 0.00 & 1.00 \\
Graduation (within faculty) & 16931 & 0.49 & 0.50 & 0.00 & 1.00 \\
Final grade & 8204 & 2.97 & 0.59 & 1.00 & 4.00 \\
Male & 12315 & 0.47 & 0.50 & 0.00 & 1.00 \\
Private health insurance & 12315 & 0.22 & 0.42 & 0.00 & 1.00 \\
Purchasing power index & 12315 & 98.50 & 11.79 & 64.72 & 186.99 \\
Theology & 16931 & 0.02 & 0.13 & 0.00 & 1.00 \\
Law & 16931 & 0.07 & 0.26 & 0.00 & 1.00 \\
Medicine & 16931 & 0.09 & 0.28 & 0.00 & 1.00 \\
Humanities & 16931 & 0.20 & 0.40 & 0.00 & 1.00 \\
Mathematics & 16931 & 0.04 & 0.19 & 0.00 & 1.00 \\
Physics & 16931 & 0.03 & 0.18 & 0.00 & 1.00 \\
Chemistry & 16931 & 0.04 & 0.19 & 0.00 & 1.00 \\
Geology/Geography & 16931 & 0.03 & 0.18 & 0.00 & 1.00 \\
Biology & 16931 & 0.08 & 0.28 & 0.00 & 1.00 \\
Forest sciences & 16931 & 0.04 & 0.19 & 0.00 & 1.00 \\
Agriculture & 16931 & 0.09 & 0.29 & 0.00 & 1.00 \\
Economic sciences & 16931 & 0.16 & 0.37 & 0.00 & 1.00 \\
Social sciences & 16931 & 0.11 & 0.31 & 0.00 & 1.00 \\
\hline \hline
\end{tabular}

Grades transformed to $1-4$ scale, with 4 being the best grade and 1 being the worst grade that is still a pass. 
The mean university GPA is 2.97 and hence, higher than the mean high school GPA of all students in the dataset which is 2.50. Furthermore, the standard deviation of the final university grade is smaller than the standard deviation of the high school GPA. This indicates that compared to the grade of the high school leaving certificate, the distribution of the final university grade is compressed and shifted to the upper end of the grading scale.

With regard to the other covariates, we see that $47 \%$ of the students are male and $22 \%$ hold a private health insurance. The mean purchasing power index is 98.50 , meaning that the mean purchasing power in our sample is $1.5 \%$ lower than the German average.

Taking a look at the distribution of students across faculties, we see that the highest share of students is studying at the faculty of humanities (20\%). Theology, on the other hand, is the smallest faculty with a share of $2 \%$.

\subsubsection{Empirical setup}

We start by examining the broadest measure of academic success, namely, whether or not a student graduates from university at all. Afterwards, we narrow our view towards graduation within fields, considering a change of field as a failure in the abandoned subject. Finally, we focus on the final grade of the university degree. This grade is a measure of the relative success within the group of successful students completing their studies.

For each of the three outcome variables we start with the GPA achieved at high school as independent variable only and continue by adding the full set of controls. These also include indicator variables for all 16 German states excluding Lower Saxony, the state where Goettingen is located, so as to reflect potential differences between the states concerning schooling systems and grading standards. Afterwards, we allow for differing effects by faculties. The binary outcome, graduation, is analyzed using probit models. For the continuous outcome variable, university grade, we use simple OLS models. In all the regressions we cluster standard errors by administrative district.

In order to interpret the regression results of the probit models right away, we 
display marginal effects for a benchmark student. ${ }^{15}$ For categorical variables the effects are calculated as discrete changes from the base category. Our benchmark student is characterized by the average high school leaving grade and income, and the mode of categorical variables. Accordingly, the student is female, holds a public health insurance and finished high school in Lower Saxony.

\subsection{Results}

There is a strong ex ante expectation that the better the high school leaving grade is, the better the performance at university should be. High income as well as a private health insurance status are expected to have positive effects on academic success. Low family income, proxied by the purchasing power index, might inhibit academic success through channels different from performance in high school. Students from low income families might lack sufficient monetary support and thus have to earn their living expenses outside university, such as working in bars, shops or factories, and thus would have less time to study. They might be less able to buy books that are not (numerous) in the libraries or other auxiliary devices such as software packages. However, payments according to the Federal Training Assistance Act $(B A f \ddot{o} G)$ should at least partly counteract this effect by providing financial support for students from poorer families. ${ }^{16}$ We do not have a clear ex ante expectation about the influence of gender and the different faculties.

\subsubsection{University level}

Table 3.2 shows the expected highly significant and positive effect of the high school leaving grade on academic success. A marginal improvement of this grade increases the probability of the benchmark student to graduate at all from university by about 21 percentage points per grade, and within fields by about 16 percentage points. An

\footnotetext{
${ }^{15}$ The coefficients of the probit regressions can be found in Tables 3.A.1-3.A.3.b in Appendix 3.A.II.

${ }^{16}$ These payments are based on the income of the parents and the student. They can amount to up to 670 euros per month (2010) of which only $50 \%$ are to be repaid, capped at a maximum amount due of 10,000 euros. In winter term $2009 / 2010$ almost $20 \%$ of all students in Goettingen received payments according to this act.
} 
Table 3.2: University level

\begin{tabular}{|c|c|c|c|c|c|c|}
\hline \multirow[t]{2}{*}{ Dependent Variable: } & \multicolumn{2}{|c|}{$\begin{array}{c}\text { Graduation } \\
\text {-All faculties- } \\
\text { Probit }\end{array}$} & \multicolumn{2}{|c|}{$\begin{array}{c}\text { Graduation } \\
\text {-Within faculty- } \\
\text { Probit }\end{array}$} & \multicolumn{2}{|c|}{$\begin{array}{c}\text { Final Grade } \\
\text { OLS }\end{array}$} \\
\hline & (1) & (2) & (3) & (4) & (5) & $(6)$ \\
\hline High school GPA & $\begin{array}{c}0.210^{* * *} \\
(28.121)\end{array}$ & $\begin{array}{c}0.210^{* * *} \\
(28.444)\end{array}$ & $\begin{array}{c}0.165^{* * *} \\
(21.810)\end{array}$ & $\begin{array}{c}0.161^{* * *} \\
(26.022)\end{array}$ & $\begin{array}{c}0.371^{* * *} \\
(0.010)\end{array}$ & $\begin{array}{c}0.386^{* * *} \\
(0.010)\end{array}$ \\
\hline Male & & $\begin{array}{c}-0.006 \\
(-0.548)\end{array}$ & & $\begin{array}{c}-0.009 \\
(-1.077)\end{array}$ & & $\begin{array}{l}-0.019 \\
(0.014)\end{array}$ \\
\hline Private health insurance & & $\begin{array}{c}0.053^{* * *} \\
(4.825)\end{array}$ & & $\begin{array}{c}0.036^{* * *} \\
(3.826)\end{array}$ & & $\begin{array}{c}0.014 \\
(0.015)\end{array}$ \\
\hline Purchasing power index & & $\begin{array}{c}0.001 \\
(0.748)\end{array}$ & & $\begin{array}{c}0.000 \\
(0.423)\end{array}$ & & $\begin{array}{c}0.001 \\
(0.001)\end{array}$ \\
\hline Constant & & & & & $\begin{array}{c}1.986^{* * *} \\
(0.027)\end{array}$ & $\begin{array}{c}1.902^{* * *} \\
(0.070)\end{array}$ \\
\hline States included & No & Yes & No & Yes & No & Yes \\
\hline $\mathrm{R}^{2}$ & & & & & 0.155 & 0.169 \\
\hline Pseudo-R ${ }^{2}$ & 0.048 & 0.051 & 0.031 & 0.033 & & \\
\hline Log Likelihood & -8120 & -8093 & -11368 & -11338 & & \\
\hline Observations & 12315 & 12315 & 16931 & 16931 & 8204 & 8204 \\
\hline
\end{tabular}

Columns 1-4: marginal effects for benchmark student, z-statistic in parentheses; columns 5-6: coefficients, standard errors in parentheses; clustered by counties; ${ }^{*} p<0.05,{ }^{* *} p<0.01,{ }^{* * *} p<0.001$. 
improvement of the high school leaving certificate by one full grade is associated with an improvement of the expected final grade by slightly below 0.4 grades.

The controls are of lesser importance: All else being equal, coming from a family that provides a student with private health insurance increases the estimated probability of the benchmark student of graduating at all or within a faculty by 5 or 4 percentage points respectively. This effect is highly significant but relatively small: Being privately insured raises the graduation probability by as much as having a 0.25 better grade at high school. Conditional on graduating, there is no significant effect of the health insurance on the final grade.

The income variable does not show significant effects in any of the regressions presented in Table 3.2. This might indicate that financial aid, provided according to the Federal Training Assistance Act, is performing well. It could also mean that income alone is not very important for academic success if aspects such as the educational family background, as captured by the health insurance status, are accounted for. Another explanation could be that those who are negatively affected by their low family income have never even started university education in the first place.

Finally, the higher importance of the high school leaving GPA with respect to overall graduation compared to graduation within a field might indicate that being a good (high school) student does not help to find the most preferred field of study right away. Obviously, re-orientation at an early stage of the studies towards a field that fits the student's own preferences or abilities better should not be seen as severe as an overall failure to graduate. This is especially true with respect to international comparisons. For instance in the U.S. a major might be chosen only after trying several fields whereas in Germany students select their field prior to entering university.

\subsubsection{Faculties}

Some students change their field of study while being enrolled. This might reflect some change in their preferences or time needed to search for the perfect match. At the same time it might also reflect differences in the (perceived) degree of difficulty to 
graduate or to get a good grade. Every now and then a discussion arises in Germany about whether or not some faculties give good grades too easily. The faculties in question will usually defend themselves by pointing out the high ability of their student body (see for instance Krass and Scherf, 2012). In order to address this issue, we allow for differing effects by faculties. Firstly, we add indicator variables for the 13 faculties excluding the base category/faculty, humanities. Afterwards we present separate regressions for each of the faculties.

Column (1) of Table 3.3 shows marginal effects for a probit regression, estimating the probability of graduation, for the benchmark student. Column (2) presents corresponding OLS results for the final university grade given graduation.

Many indicator variables of faculties show effects that are significant at the 0.1 percent level. For the benchmark student the predicted probability of graduating, given she started studying at the faculty of humanities, is about $39 \%$; given successful graduation, her expected final grade is 3.1. A male student is almost 2 percentage points less likely to graduate within the given faculty compared to the benchmark. Ceteris paribus, if he does, he receives slightly better grades. The private health insurance status is associated with both better grades and a higher probability of graduating.

All else being equal, the predicted probability of graduating at the faculty of economic sciences is about 19 percentage points higher than at the faculty of humanities; at the faculty of mathematics it is 6 percentage points lower than at the base faculty. Given graduation, the faculty of economic sciences awards, ceteris paribus, a final grade that is more than 0.4 grades worse than the respective grade at the faculty of humanities. This difference is greater than the expected change in the degree associated with an improvement of the high school leaving certificate by one full grade. The worst grades are awarded by the faculty of law. ${ }^{17}$

Doing the same regressions separately by faculties, the picture gets more differentiated. Tables 3.4.a and 3.4.b reveal strong differences with respect to how important the high school GPA is for the probability of graduating at the different faculties of Goettingen University. The effect is not significantly different from zero

\footnotetext{
${ }^{17}$ The faculty of law is traditionally known to only rarely award very good grades. Accordingly, not too much attention should be given to this fact.
} 
Table 3.3: Faculties

\begin{tabular}{|c|c|c|}
\hline Dependent Variable: & $\begin{array}{c}\text { Graduation } \\
\text { Probit } \\
(1)\end{array}$ & $\begin{array}{c}\text { Final Grade } \\
\text { OLS } \\
(2)\end{array}$ \\
\hline High school GPA & $\begin{array}{c}0.190^{* * *} \\
(25.212)\end{array}$ & $\begin{array}{c}0.373^{* * *} \\
(0.011)\end{array}$ \\
\hline Male & $\begin{array}{l}-0.016^{*} \\
(-2.000)\end{array}$ & $\begin{array}{c}0.049^{* * *} \\
(0.012)\end{array}$ \\
\hline Private health insurance & $\begin{array}{c}0.047^{* * *} \\
(5.040)\end{array}$ & $\begin{array}{l}0.023 * \\
(0.011)\end{array}$ \\
\hline Purchasing power index & $\begin{array}{c}0.000 \\
(0.638)\end{array}$ & $\begin{array}{l}0.001^{*} \\
(0.001)\end{array}$ \\
\hline Theology & $\begin{array}{c}-0.073^{* *} \\
(-2.580)\end{array}$ & $\begin{array}{c}-0.648^{* * *} \\
(0.086)\end{array}$ \\
\hline Law & $\begin{array}{c}-0.004 \\
(-0.233)\end{array}$ & $\begin{array}{c}-1.164^{* * *} \\
(0.024)\end{array}$ \\
\hline Medicine & $\begin{array}{c}0.075^{* *} \\
(2.989)\end{array}$ & $\begin{array}{c}-0.267^{* * *} \\
(0.024)\end{array}$ \\
\hline Mathematics & $\begin{array}{c}-0.060 * * * \\
(-3.504)\end{array}$ & $\begin{array}{c}-0.123^{* * *} \\
(0.030)\end{array}$ \\
\hline Physics & $\begin{array}{r}-0.059 * * \\
(-2.820)\end{array}$ & $\begin{array}{c}0.020 \\
(0.029)\end{array}$ \\
\hline Chemistry & $\begin{array}{l}-0.020 \\
(-0.946)\end{array}$ & $\begin{array}{c}0.042 \\
(0.033)\end{array}$ \\
\hline Geology/Geography & $\begin{array}{c}0.103^{* * *} \\
(4.959)\end{array}$ & $\begin{array}{c}0.073^{*} \\
(0.032)\end{array}$ \\
\hline Biology & $\begin{array}{c}0.119 * * * \\
(7.784)\end{array}$ & $\begin{array}{c}0.063^{* * *} \\
(0.019)\end{array}$ \\
\hline Forest sciences & $\begin{array}{c}0.283^{* * *} \\
(13.612)\end{array}$ & $\begin{array}{c}-0.327 * * * \\
(0.027)\end{array}$ \\
\hline Agriculture & $\begin{array}{c}0.259 * * * \\
(15.286)\end{array}$ & $\begin{array}{c}-0.204 * * * \\
(0.021)\end{array}$ \\
\hline Economic sciences & $\begin{array}{c}0.185^{* * *} \\
(12.445)\end{array}$ & $\begin{array}{c}-0.414^{* * *} \\
(0.018)\end{array}$ \\
\hline Social sciences & $\begin{array}{c}0.066^{* * *} \\
(4.541)\end{array}$ & $\begin{array}{c}0.001 \\
(0.019)\end{array}$ \\
\hline Constant & & $\begin{array}{c}2.056^{* * *} \\
(0.065)\end{array}$ \\
\hline States included & Yes & Yes \\
\hline $\begin{array}{l}\mathrm{R}^{2} \\
\text { Pseudo } \mathrm{R}^{2} \\
\text { Log Likelihood } \\
\text { Observations }\end{array}$ & $\begin{array}{c}0.062 \\
-11005 \\
16931\end{array}$ & 0.423 \\
\hline
\end{tabular}

Column 1: marginal effects for benchmark student, z-statistics

in parentheses; column 2: coefficients, standard errors in parentheses; clustered by county; ${ }^{*} p<0.05,{ }^{* *} p<0.01$ par*nthe0.001. 
3.4.a Graduation by faculties

\begin{tabular}{|c|c|c|c|c|c|c|c|}
\hline & \multicolumn{7}{|c|}{ Dependent Variable: Graduation } \\
\hline & Theology & Law & Medicine & Humanities & Mathematics & Physics & Chemistry \\
\hline High school GPA & $0.180^{* * *}$ & $0.256^{* * *}$ & $0.285^{* * *}$ & $0.187^{* * *}$ & $0.279^{* * *}$ & $0.209^{* * *}$ & $0.285^{* * *}$ \\
\hline & $(4.558)$ & $(11.171)$ & $(9.357)$ & $(12.378)$ & $(6.412)$ & $(7.185)$ & $(9.016)$ \\
\hline Male & 0.112 & 0.007 & 0.019 & $-0.114^{* * *}$ & 0.060 & $0.110^{*}$ & 0.043 \\
\hline & (1.789) & $(0.231)$ & $(0.685)$ & $(-6.688)$ & $(1.677)$ & $(2.257)$ & $(1.004)$ \\
\hline Private health insurance & $0.184^{*}$ & 0.019 & $0.080^{* *}$ & $0.068^{* * *}$ & $0.131^{*}$ & -0.013 & 0.011 \\
\hline & $(2.507)$ & $(0.611)$ & $(2.923)$ & $(3.541)$ & $(2.464)$ & $(-0.310)$ & $(0.253)$ \\
\hline Purchasing power index & $\begin{array}{c}0.002 \\
(0.633)\end{array}$ & $\begin{array}{c}-0.000 \\
(-0.031)\end{array}$ & $\begin{array}{c}-0.001 \\
(-0.729)\end{array}$ & $\begin{array}{c}0.002 \\
(1.522)\end{array}$ & $\begin{array}{c}-0.001 \\
(-0.575)\end{array}$ & $\begin{array}{c}-0.000 \\
(-0.246)\end{array}$ & $\begin{array}{c}0.001 \\
(0.349)\end{array}$ \\
\hline States included & Yes & Yes & Yes & Yes & Yes & Yes & Yes \\
\hline Pseudo $\mathrm{R}^{2}$ & 0.109 & 0.076 & 0.125 & 0.059 & 0.164 & 0.111 & 0.137 \\
\hline Log Likelihood & -167 & -774 & -896 & -2128 & -367 & -345 & -378 \\
\hline Observations & 284 & 1246 & 1481 & 3342 & 660 & 567 & 644 \\
\hline
\end{tabular}

Marginal effects for benchmark student, $\mathrm{z}$-statistics in parentheses; clustered by county; ${ }^{*} p<0.05,{ }^{* *} p<0.01,{ }^{* * *} p<0.001$

3.4.b Graduation by faculties

\begin{tabular}{|c|c|c|c|c|c|c|}
\hline & \multicolumn{6}{|c|}{ Dependent Variable: Graduation } \\
\hline High school GPA & 0.069 & $0.176^{* * *}$ & $0.152^{* * *}$ & $0.132^{* * *}$ & $0.159^{* * *}$ & $0.086^{* * *}$ \\
\hline & $(1.875)$ & $(8.304)$ & $(3.971)$ & $(5.451)$ & $(8.061)$ & $(4.521)$ \\
\hline Male & $-0.127^{*}$ & -0.016 & 0.031 & 0.049 & -0.022 & -0.027 \\
\hline Private health insurance & 0.061 & 0.037 & 0.040 & -0.038 & $0.064^{* *}$ & 0.011 \\
\hline & $(1.070)$ & $(1.113)$ & $(1.056)$ & $(-1.071)$ & $(3.110)$ & $(0.388)$ \\
\hline Purchasing power index & $\begin{array}{c}0.004 \\
(1.436)\end{array}$ & $\begin{array}{c}-0.002 \\
(-1.826)\end{array}$ & $\begin{array}{c}-0.000 \\
(-0.029)\end{array}$ & $\begin{array}{c}-0.002 \\
(-1.390)\end{array}$ & $\begin{array}{c}0.001 \\
(0.553)\end{array}$ & $\begin{array}{c}0.004^{* * * *} \\
(3.340)\end{array}$ \\
\hline Log Likelihood & -360 & -923 & -425 & -1004 & -1819 & -1198 \\
\hline Observations & 542 & 1410 & 666 & 1546 & 2740 & 1778 \\
\hline
\end{tabular}

Marginal effects for benchmark student, z-statistics in parentheses; clustered by county; ${ }^{*} p<0.05,{ }^{* *} p<0.01,{ }^{* * *} p<0.001$.

at the faculty of geology and geography, and it is strongest at the medical school and the faculty of chemistry. For the benchmark student at these two faculties, a marginal increase in the GPA earned in high school is associated with an increase in the graduation probability by almost 29 percentage points per grade. At the faculty of social sciences, the effect is only about one third of that size.

Private health insurance status, which proxies a high socio-economic background, is significant and has a positive sign for about half of the faculties, while being insignificant for the other faculties. Purchasing power is also of little importance for the probability of graduating at the faculty level. It is significant only at the faculty of social sciences.

For illustration and further comparison of faculties, Table 3.5 provides predicted probabilities of graduation based on the estimation results underlying Tables 3.4.a 
and 3.4.b. The predictions for the benchmark student are presented in the middle column (mean high school GPA). The remaining predictions deviate from the usual benchmark by the high school GPA used. We define low and high high school GPA as the mean GPA minus two standard deviations and mean GPA plus two standard deviations respectively.

Although we do not want to put too much emphasis on these predictions, they serve to illustrate the rather large differences between faculties. The predicted probability of graduation for the benchmark student is between roughly 20 and 60 percent. Based on these predictions, a student with a low high school GPA can hardly expect to graduate at some of the faculties, such as mathematics and physics. At other faculties chances to graduate are still relatively high; the predicted probabilities for such a student are 45 and 39 percent at the faculties of agriculture and economic sciences respectively. For an otherwise identical student with a high high school GPA the predictions vary between about 50 and 80 percent.

Tables 3.6.a and 3.6.b show corresponding regression results for final grades at graduation. There is a highly significant positive effect of the high school GPA at every faculty. However, the importance of this GPA differs strongly. It is highest at the faculty of mathematics, where the expected grade at graduation is more than half a grade better for every full grade of the high school leaving certificate. At the faculty of chemistry, where the coefficient of high school GPA is the smallest, the effect is only about half that size. Given graduation, male students can expect slightly better grades than their female fellow students in about half of the faculties. The effects of health insurance status and purchasing power are indistinguishable from zero at most faculties. ${ }^{18}$

Figure 3.1 visualizes the relationship between the GPA earned at university and at high school across selected faculties. The red lines represent fitted values for female students who are publicly insured, come from a zip code area with average purchasing power and finished high school in Lower Saxony. We can notice from the

\footnotetext{
${ }^{18}$ There is a surprisingly large, highly significant, positive effect of the private health insurance status on the final grade at university at the faculty of theology. Taking this coefficient at face value, a reason for this strong effect could be that children of pastors in Germany are privately insured. However, due to the small sample size of the underlying regression, we refrain from emphasizing this finding.
} 
Table 3.5: Predicted probabilities of graduation by faculties

\begin{tabular}{lccc}
\hline \hline & \multicolumn{3}{c}{ High school GPA } \\
\cline { 2 - 4 } & Low & Mean & High \\
\hline Theology & 0.10 & 0.27 & 0.53 \\
Law & 0.14 & 0.40 & 0.72 \\
Medicine & 0.19 & 0.52 & 0.83 \\
Humanities & 0.21 & 0.42 & 0.66 \\
Mathematics & 0.04 & 0.24 & 0.67 \\
Physics & 0.05 & 0.21 & 0.54 \\
Chemistry & 0.06 & 0.30 & 0.69 \\
Geology/Geography & 0.41 & 0.50 & 0.59 \\
Biology & 0.30 & 0.51 & 0.72 \\
Forest sciences & 0.38 & 0.57 & 0.75 \\
Agriculture & 0.45 & 0.62 & 0.77 \\
Economic sciences & 0.39 & 0.59 & 0.77 \\
Social sciences & 0.34 & 0.45 & 0.56 \\
\hline \hline
\end{tabular}

Predicted probability of graduating at a faculty for female students who are publicly insured, come from a zip code area with average purchasing power, and finished high school in Lower average purchasing power, and fished high school in Lower GPA minus two standard deviations and mean GPA plus two standard deviations, respectively. 
3.6.a Grades by faculties

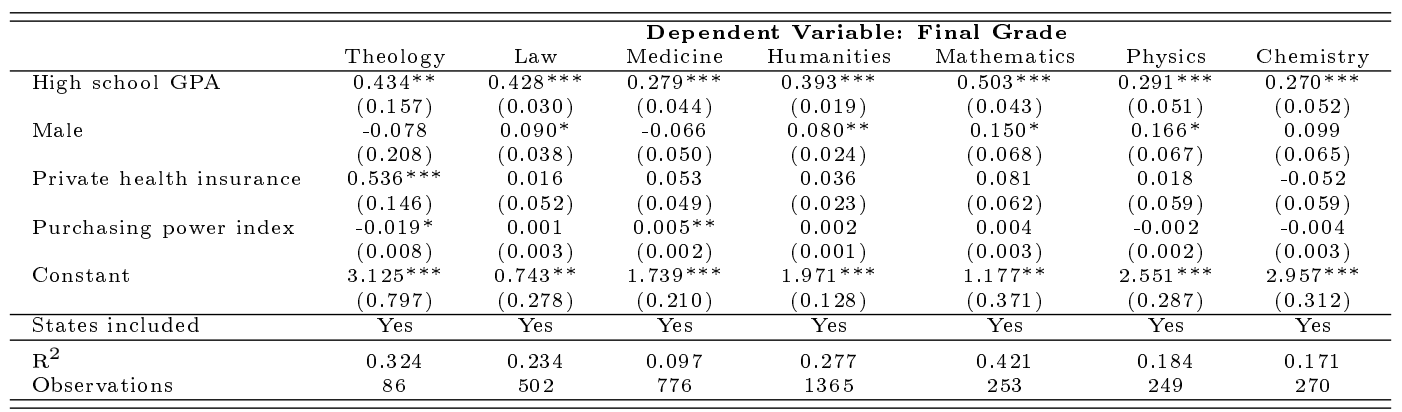

Coefficients, standard errors in parentheses; clustered by county; ${ }^{*} p<0.05,{ }^{* *} p<0.01,{ }^{* * *} p<0.001$.

3.6.b Grades by faculties

\begin{tabular}{|c|c|c|c|c|c|c|}
\hline & \multicolumn{6}{|c|}{ Dependent Variable: Final Grade } \\
\hline & Geology/Geography & Biology & Forest sciences & Agriculture & Economic sciences & Social sciences \\
\hline High school GPA & $\begin{array}{c}0.293^{* * *} \\
(0.057)\end{array}$ & $\begin{array}{c}0.288^{* * *} \\
(0.029)\end{array}$ & $\begin{array}{c}0.352^{* * *} \\
(0.041)\end{array}$ & $\begin{array}{c}0.386^{* * *} \\
(0.024)\end{array}$ & $\begin{array}{c}0.398^{* * *} \\
(0.019)\end{array}$ & $\begin{array}{c}0.398^{* * *} \\
(0.026)\end{array}$ \\
\hline Male & $\begin{array}{l}-0.064 \\
(0.051)\end{array}$ & $\begin{array}{c}0.113^{* * *} * \\
(0.033)\end{array}$ & $\begin{array}{c}0.116^{* *} \\
(0.043)\end{array}$ & $\begin{array}{c}0.018 \\
(0.029)\end{array}$ & $\begin{array}{c}0.017 \\
(0.022)\end{array}$ & $\begin{array}{c}0.054 \\
(0.027)\end{array}$ \\
\hline Private health insurance & $\begin{array}{c}0.011 \\
(0.053)\end{array}$ & $\begin{array}{c}0.013 \\
(0.030)\end{array}$ & $\begin{array}{c}0.041 \\
(0.053)\end{array}$ & $\begin{array}{c}-0.087^{*} \\
(0.037)\end{array}$ & $\begin{array}{c}0.014 \\
(0.035)\end{array}$ & $\begin{array}{c}0.050 \\
(0.035)\end{array}$ \\
\hline Purchasing power index & $\begin{array}{l}-0.003 \\
(0.003)\end{array}$ & $\begin{array}{c}0.002 \\
(0.001)\end{array}$ & $\begin{array}{c}0.003 \\
(0.002)\end{array}$ & $\begin{array}{l}-0.000 \\
(0.002)\end{array}$ & $\begin{array}{c}0.001 \\
(0.001)\end{array}$ & $\begin{array}{c}0.000 \\
(0.002)\end{array}$ \\
\hline Constant & $\begin{array}{c}2.807^{* * *} * \\
(0.335) \\
\end{array}$ & $\begin{array}{c}2.339^{* * *} \\
(0.159) \\
\end{array}$ & $\begin{array}{c}1.573^{* * *} \\
(0.250) \\
\end{array}$ & $\begin{array}{c}2.030^{* * *} \\
(0.171)\end{array}$ & $\begin{array}{c}1.575^{* * *} \\
(0.129)\end{array}$ & $\begin{array}{c}2.110^{* * *} \\
(0.171)\end{array}$ \\
\hline States included & Yes & Yes & Yes & Yes & Yes & Yes \\
\hline $\begin{array}{l}\mathrm{R}^{2} \\
\text { Observations }\end{array}$ & $\begin{array}{c}0.212 \\
250\end{array}$ & $\begin{array}{c}0.158 \\
784\end{array}$ & $\begin{array}{c}0.184 \\
408\end{array}$ & $\begin{array}{c}0.232 \\
953\end{array}$ & $\begin{array}{l}0.247 \\
1534\end{array}$ & $\begin{array}{l}0.250 \\
774\end{array}$ \\
\hline
\end{tabular}

Coefficients, standard errors in parentheses; clustered by county; ${ }^{*} p<0.05,{ }^{* *} p<0.01,{ }^{* * *} p<0.001$. 
upper two panels of this figure that grades in humanities are generally better than in economic sciences. The lower two panels show that the relationship between high school GPA and university grade is much steeper in mathematics than in biology.

Figure 3.1: Grades at selected faculties
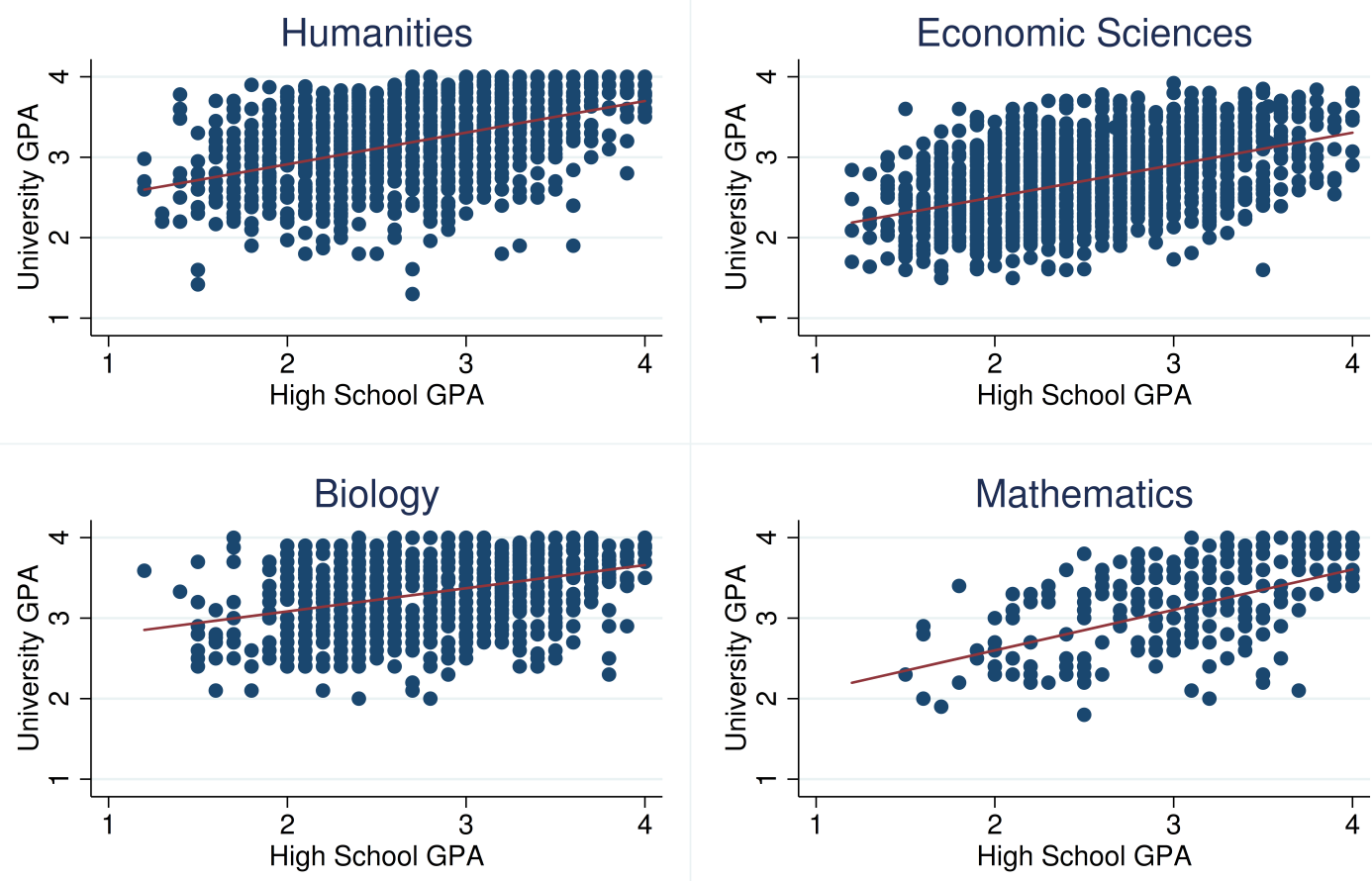

$$
\text { - University GPA } \quad \text { fitted values }
$$

Dots represent one or several observations. Fitted values are the predicted university GPA for female students who are publicly insured, come from a zip code area with average purchasing power, and finished high school in Lower Saxony.

Comparing the faculties with the highest number of students, humanities and economic sciences, it seems to be easier to graduate in economic sciences whereas the expected grade conditional on graduation is worse. This pattern can also be found for a couple of other faculties and might suggest differences in grading and examination culture between the faculties. It seems that at some faculties it is more difficult to obtain a degree while the grades given differentiate less strongly between 
students. However, at others achieving a degree is more likely while the grades obtained vary more within the grading scale.

There are a number of possible mechanisms which might contribute to these faculty-specific results. Firstly, students may self-select into faculties on unobservable characteristics related to the outcome variables. For example, some students may be more motivated to obtain good grades at university than they were in high school. If such students disproportionately choose humanities rather than mathematics or economics, we will find better grades in the former faculty conditional on high school GPA. While we cannot exclude such self-selection with the data at hand, in our view it is not very plausible that students of various faculties should differ precisely in this respect.

Alternatively, and arguably more convincingly, the results may be driven by features of the teaching and grading system in the respective faculties. A first explanation along this line is based on the similarity between curricula in high school and in university. The high school grade is a composite of a comprehensive variety of subjects whereas university studies are more specialized. Since students likely choose subjects which fit their specific abilities, one may expect that in highly specialized fields, university grades are better and less closely associated with high school GPA than in broader subjects. Given that the impact of high school GPA on university grades is largest in mathematics, which is a more specialized field than social science or economics, this explanation, however, does not find much support in the data.

Instead, the differences in grades are likely to reflect different grading cultures. Some faculties may simply be willing to award good grades to most students without differentiating strongly among good and mediocre performance. More subtly, an upward drift of average grades may be built in the structure of some degree programs. When a program grants ample choice among electives, students can avoid difficult or unpleasant courses while still obtaining the degree. Moreover, if students can freely choose courses, teachers might have an incentive to attract students by grading leniently. As a result, grades from such a program will be compressed at the upper end of the scale compared to programs with a more rigid structure of compulsory courses. 
Although we have some sympathy for the last explanation, our data do not permit to conclusively distinguish between these mechanisms. Instead, we confine ourselves to pointing out the main result of this chapter: The relationship between high school grades and university success varies in a statistically discernible manner among faculties, which hints at some differences in grading, teaching, and examination cultures.

\subsection{Discussion and policy implications}

In this chapter, the determinants of studying successfully are analyzed using data from more than 12,000 students from Goettingen University. Two main results are shown. Firstly, the high school leaving grade is by far the best predictor of both the probability of graduating and the final grade obtained at university. Other factors, notably gender or social origin, play only a minor role. Secondly, differences emerge among the various faculties regarding grading and graduation policies. In some faculties, like humanities or social sciences, the rate of graduation is low but those who graduate can expect to obtain quite good grades even when they start from a weaker academic base as measured by the high school GPA. In other faculties, such as economic sciences or forest sciences, the chance of obtaining a degree is relatively high whereas grades are moderate, and strongly linked to high school GPA. Finally, in some faculties such as mathematics and physics, graduation appears to be very difficult and good grades are hard to obtain, especially for weaker students.

These findings carry a number of implications both for the university and for the students individually as well as for education policy in general. Most obviously, our results support the current process of admission to German universities, which is based primarily on high school GPA. Clearly, this practice contributes to improving the academic success of those admitted. We do not find any evidence that adding other information can improve the selection. Specifically, variables capturing income or social background have a comparatively low explanatory power. This suggests that granting privileged access to minorities or providing universities with financial incentives to admit more students from poor districts, rather than focusing exclusively on ability, may raise the number of unsuccessful students. Most of the impact 
of social origin on university achievement is already absorbed in the high school leaving grade. Consequently, policy should address social imbalances in educational outcomes at earlier stages of the academic career.

For prospective students, the faculty specific results, summarized in Table 3.5, may give useful hints about what subject to choose. A student with mean high school GPA has a higher chance of graduating if she chooses agriculture or economic sciences rather than humanities or social sciences. If obtaining some degree irrespective of the field is very important for her, such a student should enroll in the former rather than in the latter faculties. Considering mathematics, physics, or chemistry, the recommendation is even clearer: The average student will graduate in these faculties with a probability of $30 \%$ or less. For weaker students with high school GPA substantially below the mean this probability falls below $10 \%$. This suggests that these three fields are almost unfeasible for students in the bottom half of the ability distribution and that such students are well advised to opt for other fields.

Extending the principle of selection on academic merit to the aggregate level obviously raises a consistency issue: Not every university or field can be restricted to the best students, since the weaker ones also will have to be placed somewhere, or else must be told not to study. This points out a basic choice which education policy must make: Should universities provide an excellent education for the most able individuals at a level defined by the current state of knowledge, or should tertiary education be targeted to large numbers of students and settle for an academic level accessible for these? Related to this, there are competing views on the main purpose of university studies. On one hand, in Humboldt's tradition, one may see academic studies mainly as a tool of personal intellectual enhancement, where knowledge, understanding and academic debate are rewards in themselves. On the other hand, studies may be seen as an investment in productivity, whose main reward comes in the form of a higher wage. In the former view, graduation and examination grades are of lesser importance. In the latter case, the signaling value of a degree is likely to be essential for employers. As a consequence, the labor market will honor only completed degrees, and a wage premium will be paid for good grades as long as these are rare enough so as to convey credible information. 
The results presented in this chapter suggest that faculties take different sides in this debate. In humanities, graduation rates are relatively low and individual grades are less differentiated than in other fields. This corresponds to the idea that one does not study for the sake of the examination or for a higher wage, but for intrinsic motivation. Quite possibly these fields specifically attract students with such expectations. In this view, a low completion rate in such subjects should not be seen as a sign of failure. These fields offer students an education tailored to their abilities and preferences and students use this offer to the extent which is individually optimal. On the other end of the scale, examinations in mathematics, physics and chemistry are highly selective. Thereby, these fields cannot cater to large numbers of students, but they prepare those who make it for demanding sections of the labor market. Similarly, economic sciences serve the labor market by awarding differentiated grades while still being accessible for large numbers of weaker students.

These considerations shed some light on the recommendation, repeatedly voiced by the OECD (see for instance OECD, 2013, p. 151), that Germany should produce more university graduates and the corresponding complaint by employers' organizations that German industry faces a shortage of graduates from mathematics, natural sciences, and engineering (see Anger et al., 2013). It is certainly conceivable that reforms in secondary schooling can raise the number of students entering university. It appears far-fetched, however, that a large fraction of those additional students will display academic abilities superior to those of the average current student. Our results show that average or below average students will typically be unable to successfully complete a degree in mathematics, physics or chemistry. Therefore it seems highly unlikely that an increase in university enrollment will produce substantial numbers of additional graduates in the subjects required by industry, at least as long as the concerned faculties are unwilling to lower their academic standards. If this does not occur, any increase in university enrollment will lead to larger numbers of graduates in those fields which cater to the preferences and abilities of the majority of students but not in those fields which firms demand. 


\section{Appendix to Chapter 3}

\section{Appendix 3.A.I: Data processing}

We exclude students for whom not all information is available as well as students for whom we observe pure data errors, such as when the grade of the high school leaving certificate is not within the possible interval. Ph.D. students are also dropped from the dataset. The reason for this is that they form a highly selective group and their success may be influenced by other factors than regular students' performance. Furthermore, we only take into account students who either finished university with a degree or dropped out of their study program. Since students are asked to give the reason for dropping out when they leave university, we can distinguish between real drop outs and students who intend to continue their studies at another university. We exclude these students from the sample in order not to register a drop out for the latter group.

As German and foreign high school leaving grades may not be comparable and university success of students with a foreign educational background may be influenced by additional factors such as language skills, we only take into account students who hold a German high school leaving certificate. In addition, we exclude students with a high school leaving grade of 4.0, the worst grade still allowing a student to pass. This is done as in our dataset a high school leaving grade of 4.0 was often found for students, in particular for foreign students, who enrolled in fields of study without admission restriction. This strongly suggests that the grade is sometimes used as a place holder when the real grade seemed not to be important for the admission procedure. However, we are confident that we have only deleted a very small number of students who actually have a high school leaving grade of 4.0 by imposing this restriction.

In addition, students have to provide information about their home address, usually their parents' address, and their semester address, usually the place students live by themselves. Since most students move to Goettingen when starting university, home and semester address should differ. Nonetheless, for some students in our dataset the two zip-codes are identical. As we make use of the parents' address 
in our analysis it is important that the correct zip-code is used. To deal with this problem, we look at all students for whom the zip-code of their home and semester address are the same. If both zip-codes belong to a place outside of Goettingen, it is very likely that this student is still living with his or her parents. If the zip-codes are identical and from Goettingen, it might be that the student did not provide any information about his or her parents' home address. Therefore, we take a look at the administrative district the student went to school in. If he or she graduated from a high school in Goettingen, we have no reason to doubt that his or her parents also live there. On the other hand, if he or she went to school outside of Goettingen, it is not entirely clear that the information about the home address really corresponds to the parental address. Consequently, we exclude these students from the sample. 


\section{Appendix 3.A.II: Coefficients}

Table 3.A.1: University level - Coefficients for Table 3.2

\begin{tabular}{lcccc}
\hline Dependent Variable: & \multicolumn{2}{c}{$\begin{array}{c}\text { Graduation } \\
\text {-All faculties- }\end{array}$} & \multicolumn{2}{c}{$\begin{array}{c}\text { Graduation } \\
\text {-Within faculty- }\end{array}$} \\
& \multicolumn{2}{c}{ Probit } & \multicolumn{2}{c}{ Probit } \\
& $(1)$ & $(2)$ & $(3)$ & $(4)$ \\
\hline High school GPA & $0.528^{* * *}$ & $0.527^{* * *}$ & $0.414^{* * *}$ & $0.405^{* * *}$ \\
& $(0.019)$ & $(0.018)$ & $(0.019)$ & $(0.016)$ \\
Male & & -0.014 & & -0.022 \\
& & $(0.025)$ & & $(0.021)$ \\
Private health insurance & & $0.134^{* * *}$ & & $0.091^{* * *}$ \\
& & $(0.028)$ & & $(0.024)$ \\
Purchasing power index & & 0.002 & & 0.001 \\
& & $(0.002)$ & & $(0.002)$ \\
Constant & $-1.359^{* * *}$ & $-1.513^{* * *}$ & $-1.076^{* * *}$ & $-1.142^{* * *}$ \\
& $(0.048)$ & $(0.271)$ & $(0.079)$ & $(0.240)$ \\
\hline States included & No & Yes & No & Yes \\
\hline Pseudo- ${ }^{2}$ & 0.048 & 0.051 & 0.031 & 0.033 \\
Log Likelihood & -8120 & -8093 & -11368 & -11338 \\
Observations & 12315 & 12315 & 16931 & 16931 \\
\hline \hline
\end{tabular}

Coefficients, standard errors in parentheses; clustered by counties; ${ }^{*} p<0.05,{ }^{* *} p<0.01$, $* * * p<0.001$ 
Table 3.A.2: Faculties - Coefficients for Table 3.3

\begin{tabular}{|c|c|}
\hline Dependent Variable: & $\begin{array}{c}\text { Graduation } \\
\text { Probit } \\
(1)\end{array}$ \\
\hline High school GPA & $\begin{array}{c}0.493^{* * *} \\
(0.016)\end{array}$ \\
\hline Male & $\begin{array}{l}-0.040^{*} \\
(0.020)\end{array}$ \\
\hline Private health insurance & $\begin{array}{c}0.119 * * * \\
(0.023)\end{array}$ \\
\hline Purchasing power index & $\begin{array}{c}0.001 \\
(0.002)\end{array}$ \\
\hline Theology & $\begin{array}{l}-0.196^{*} \\
(0.080)\end{array}$ \\
\hline Law & $\begin{array}{c}-0.009 \\
(0.039)\end{array}$ \\
\hline Medicine & $\begin{array}{c}0.191^{* *} \\
(0.065)\end{array}$ \\
\hline Humanities & base \\
\hline Mathematics & $\begin{array}{c}-0.160^{* * *} \\
(0.047)\end{array}$ \\
\hline Physics & $\begin{array}{c}-0.157^{* *} \\
(0.057)\end{array}$ \\
\hline Chemistry & $\begin{array}{l}-0.052 \\
(0.055)\end{array}$ \\
\hline Geology/Geography & $\begin{array}{c}0.261^{* * *} \\
(0.052)\end{array}$ \\
\hline Biology & $\begin{array}{c}0.302^{* * *} \\
(0.039)\end{array}$ \\
\hline Forest sciences & $\begin{array}{c}0.730 * * * \\
(0.058)\end{array}$ \\
\hline Agriculture & $\begin{array}{c}0.663^{* * * *} \\
(0.046)\end{array}$ \\
\hline Economic sciences & $\begin{array}{c}0.467^{* * *} \\
(0.038)\end{array}$ \\
\hline Social sciences & $\begin{array}{c}0.167^{* * *} \\
(0.037)\end{array}$ \\
\hline Constant & $\begin{array}{c}-1.587^{* * * *} \\
(0.216)\end{array}$ \\
\hline States included & Yes \\
\hline $\begin{array}{l}\text { Pseudo } \mathrm{R}^{2} \\
\text { Log Likelihood } \\
\text { Observations }\end{array}$ & $\begin{array}{c}0.062 \\
-11005 \\
16931\end{array}$ \\
\hline
\end{tabular}

Coefficients, standard errors in parentheses clustered by county; ${ }^{*} p<0.05,{ }^{* *} p<0.01$,
${ }^{* * *} p<0.001$. 
3.A.3.a Graduation by faculties - Coefficients for Table 3.4.a

\begin{tabular}{lccccccc}
\hline \hline & \multicolumn{7}{c}{ Dependent Variable: Graduation } \\
& Theology & Law & Medicine & Humanities & Mathematics & Physics & Chemistry \\
\hline High school GPA & $0.539^{* * *}$ & $0.663^{* * *}$ & $0.714^{* * *}$ & $0.479^{* * *}$ & $0.889^{* * *}$ & $0.717^{* * *}$ & $0.820^{* * *}$ \\
& $(0.115)$ & $(0.057)$ & $(0.076)$ & $(0.038)$ & $(0.083)$ & $(0.088)$ & $(0.089)$ \\
Male & 0.310 & 0.019 & 0.048 & $-0.304^{* * *}$ & 0.181 & $0.336^{*}$ & 0.121 \\
& $(0.167)$ & $(0.084)$ & $(0.070)$ & $(0.047)$ & $(0.112)$ & $(0.150)$ & $(0.120)$ \\
Private health insurance & $0.496^{* *}$ & 0.048 & $0.204^{* *}$ & $0.173^{* * *}$ & $0.373^{* *}$ & -0.044 & 0.032 \\
& $(0.188)$ & $(0.078)$ & $(0.071)$ & $(0.048)$ & $(0.139)$ & $(0.145)$ & $(0.125)$ \\
Purchasing power index & 0.006 & -0.000 & -0.003 & 0.005 & -0.003 & -0.001 & 0.002 \\
& $(0.009)$ & $(0.004)$ & $(0.003)$ & $(0.003)$ & $(0.006)$ & $(0.006)$ & $(0.005)$ \\
Constant & $-2.518^{* *}$ & $-1.900^{* * *}$ & $-1.496^{* * *}$ & $-1.848^{* * *}$ & $-2.571^{* * *}$ & $-2.438^{* * *}$ & $-2.742^{* * *}$ \\
& $(0.953)$ & $(0.419)$ & $(0.406)$ & $(0.364)$ & $(0.623)$ & $(0.666)$ & $(0.551)$ \\
\hline States included & Yes & Yes & Yes & Yes & Yes & Yes & Yes \\
\hline Pseudo R & 0.109 & 0.076 & 0.125 & 0.059 & 0.164 & 0.111 & 0.137 \\
Log Likelihood & -167 & -774 & -896 & -2128 & -367 & -345 & -378 \\
Observations & 284 & 1246 & 1481 & 3342 & 660 & 567 \\
\hline
\end{tabular}

Coefficients, standard errors in parentheses; clustered by county; ${ }^{*} p<0.05,{ }^{* *} p<0.01,{ }^{* * *} p<0.001$.

3.A.3.b Graduation by faculties - Coefficients for Table 3.4.b

\begin{tabular}{|c|c|c|c|c|c|c|}
\hline & \multicolumn{6}{|c|}{ Dependent Variable: Graduation } \\
\hline & Geology/Geography & Biology & Forest sciences & Agriculture & Economic sciences & Social sciences \\
\hline High school GPA & $\begin{array}{c}0.172 \\
(0.092)\end{array}$ & $\begin{array}{c}0.441^{* * *} \\
(0.053)\end{array}$ & $\begin{array}{c}0.388^{* * *} \\
(0.104)\end{array}$ & $0.346^{* * *}$ & $\begin{array}{c}0.410^{* * *} \\
(0.049)\end{array}$ & $\begin{array}{c}0.218^{* * *} \\
(0.048)\end{array}$ \\
\hline Male & $\begin{array}{l}-0.325^{*} \\
(0.151)\end{array}$ & $\begin{array}{c}-0.040 \\
(0.069)\end{array}$ & $\begin{array}{c}0.081 \\
(0.123)\end{array}$ & $\begin{array}{c}0.132 \\
(0.090)\end{array}$ & $\begin{array}{l}-0.057 \\
(0.053)\end{array}$ & $\begin{array}{c}-0.069 \\
(0.056)\end{array}$ \\
\hline Private health insurance & $\begin{array}{c}0.154 \\
(0.145)\end{array}$ & $\begin{array}{c}0.093 \\
(0.083)\end{array}$ & $\begin{array}{c}0.102 \\
(0.097)\end{array}$ & $\begin{array}{l}-0.098 \\
(0.092)\end{array}$ & $\begin{array}{l}0.168^{* *} \\
(0.055)\end{array}$ & $\begin{array}{c}0.028 \\
(0.072)\end{array}$ \\
\hline Purchasing power index & $\begin{array}{c}0.010 \\
(0.007)\end{array}$ & $\begin{array}{c}-0.006 \\
(0.003)\end{array}$ & $\begin{array}{l}-0.000 \\
(0.005)\end{array}$ & $\begin{array}{l}-0.005 \\
(0.004)\end{array}$ & $\begin{array}{c}0.002 \\
(0.003)\end{array}$ & $\begin{array}{c}0.009^{* * *} \\
(0.003)\end{array}$ \\
\hline Constant & $\begin{array}{l}-1.375 \\
(0.715)\end{array}$ & $\begin{array}{c}-0.472 \\
(0.369)\end{array}$ & $\begin{array}{l}-0.772 \\
(0.546)\end{array}$ & $\begin{array}{c}-0.031 \\
(0.423)\end{array}$ & $\begin{array}{c}-0.971^{* *} \\
(0.374)\end{array}$ & $\begin{array}{c}-1.589^{* * *} \\
(0.308)\end{array}$ \\
\hline States included & Yes & Yes & Yes & Yes & Yes & Yes \\
\hline $\begin{array}{l}\text { Pseudo } \mathrm{R}^{2} \\
\text { Log Likelihood }\end{array}$ & $\begin{array}{c}0.039 \\
-360 \\
542\end{array}$ & $\begin{array}{l}0.047 \\
-923 \\
1410\end{array}$ & $\begin{array}{c}0.043 \\
-425 \\
666\end{array}$ & $\begin{array}{l}0.024 \\
-1004 \\
1546\end{array}$ & $\begin{array}{c}0.032 \\
-1819 \\
2740\end{array}$ & $\begin{array}{c}0.019 \\
-1198 \\
1778\end{array}$ \\
\hline
\end{tabular}

Coefficients, standard errors in parentheses; clustered by county; ${ }^{*} p<0.05,{ }^{* *} p<0.01,{ }^{* * *} p<0.001$. 


\section{Chapter 4}

\section{The Second Dividend of Studying}

\section{Abroad: \\ The Impact of International Student Mobility on Academic Performance*}

\subsection{Introduction}

Globalization and internationalization do not only influence business and political decisions, but also affect university education. According to OECD figures (OECD, 2012) the number of students studying abroad increased by about $400 \%$ between 1975 and 2010. In 2010 more than 4.1 million students in higher education attended universities outside their home country (OECD, 2012). A similar pattern can be observed in Germany: until the year 2000 the share of university students going abroad increased to one third and stayed at this level since then (DAAD and HIS, 2013). Some of these students obtain their whole degree in a foreign country, while others do an internship or a language course. However, it is noted that the most popular reason for going abroad for German students is a temporary study-related

*This chapter originates from joint work with Katharina Lerche, née Suntheim (see Meya and Suntheim, 2014). 
visit abroad. More than half of the German students who decide to go abroad choose to study at a foreign university for one or two semesters (DAAD and HIS, 2013). The popularity of such a sojourn raises the question of what gains can be expected from being internationally mobile during university education.

The most prominent benefit from studying in a foreign country is arguably personal development. Study-related visits abroad are seen to have a positive impact on students' personality and cross-cultural skills. Students who went abroad for part of their university education report to be more independent, approachable and agreeable than before their stay. Furthermore, they are more open to foreign cultures and ways of working (Clarke et al., 2009; DAAD and HIS, 2013; Zimmermann and Neyer, 2013).

International experience seems to affect job market opportunities and decisions: Parey and Waldinger (2011) point out that studying in a foreign country influences the probability of working abroad later in life. Using an instrumental variables approach, they find that internationally mobile students are 15 percentage points more likely to work abroad after graduation than their counterparts who stayed at home. As possible reasons they emphasize the importance of factors such as having a partner from another country or interest in different cultures. These results are supported by Teichler (2011) who analyzes the impact of international experience gained during or shortly after graduation on later employment. Additionally, he finds that even if graduates with international experience work in their home country, they are more likely to have a job that requires cross-cultural skills in particular. In their study regarding the relationship between studying abroad and later migration, Dreher and Poutvaara (2011) show that an increase in the number of foreign students studying in the United States also leads to higher immigration later on.

More directly looking at the success in the job-market, Di Pietro (2013) finds that a study-related visit abroad increases the probability of being employed three years after graduation by 24 percentage points. In contrast, Messer and Wolter (2007) do not find a causal effect of a study-related visit abroad on the first job salary and the probability of starting a Ph.D. once they instrument for studying abroad. They suggest that differences in later job-market success and academic careers may be due to internationally mobile students generally being more capable rather than 
due to the visit abroad.

This chapter contributes to the literature on the effects of international student mobility by focusing on the impact of a sojourn on academic performance. Drawing on a rich dataset collected at Goettingen University, Germany, we analyze if and how studying one or two semesters at a foreign institution influences the final university grade achieved and the time needed to finish the degree program. We apply a propensity score matching strategy in order to overcome a potential problem of self-selection into studying abroad. This is possible due to the unique dataset at hand containing a variety of individual information on more than 2,500 students who successfully completed their bachelor studies.

We find that a temporary study-related visit abroad on average improves the final university grade by 0.095-0.17 grades. We call this effect the second dividend of studying abroad, as it seems that it arises as a consequence of students strategically deciding which grades count towards their degree. With regard to students who studied abroad, we note that the final university grade is 0.2 grades better for those who count the grades obtained at the foreign university towards their degree in contrast to those who do not transfer any grades. Furthermore, we find that students who go abroad have a lower probability of finishing their bachelor studies within the standard time period. This further supports our interpretation that students selectively transfer grades achieved abroad which are better than the average grade achieved at the home university. Moreover, it implies that selective transferring of grades comes at a cost.

This chapter is structured as follows: Section 4.2 describes the dataset and presents summary statistics. Section 4.3 gives an overview of the empirical framework used in the analysis. Section 4.4 presents and interprets our empirical results. Section 4.5 tests the robustness of our results. Finally, Section 4.6 summarizes the main findings and concludes.

\subsection{Data description}

For our analysis we use a unique dataset consisting of administrative student data collected at Goettingen University. It is to a large extent a subset of the dataset used 
in the previous chapter and contains detailed, anonymous information about more than 2,500 students who successfully completed their bachelor studies between 2006 and 2011, such as the students' university and high school degree and grade, subjects studied, their gender, type of health insurance and the zip-code of their address during semester as well as that of their parents' residence. Information on studyrelated visits abroad is provided by the international office of Goettingen University which collects data concerning students who take part in international mobility programs such as the European Union student mobility program, ERASMUS. We also use information about exams taken at a foreign university provided by the examination office in order to register stays abroad for students who did not take part in such a program but still studied at a foreign university. ${ }^{19}$

We restrict our sample to bachelor students who started their university studies at Goettingen University. ${ }^{20}$ The reason for this is that we can then observe all examinations relevant for the degree for these students. Furthermore, for all bachelor programs at Goettingen University some common rules apply, among them, a regular length of study of six semesters including thesis. As we have detailed information on the students' course of study, we are able to take into account only semesters of the field of study the respective student achieved her bachelor degree in. Like in Chapter 3, we restrict our attention to students who hold a German high school certificate as we use the grade received to control for pre-university ability.

Since we examine the impact of a study-related visit to a foreign university (usually one or two study terms) on academic performance, we are only interested in study-related stays during which the student could take courses counting towards his or her degree at Goettingen University. Students who took part in mobility programs that also support other kinds of stays, such as internships, short term field excursions and language courses, are dropped. Furthermore, in some unusual cases, students are enrolled in more than one bachelor program at the same time. In this case it cannot clearly be identified which courses taken abroad were transferred to the respective degree. Therefore, we exclude these students from the analysis.

\footnotetext{
${ }^{19}$ See Chapter 3, especially Appendix 3.A.I for further detailed information on data collection, processing and filtering.

${ }^{20}$ Students of the faculties of theology and law are not part of the analysis, as they are only in extremely rare cases enrolled in bachelor programs.
} 
We distinguish between students who stayed abroad and counted all or some of the grades obtained towards their bachelor degree at Goettingen University and students who stayed abroad, but did not transfer any of the grades achieved at the foreign university. Although most students taking part in an international mobility program are obliged to take courses at the foreign university, these courses taken abroad might not necessarily be part of the home curriculum, and therefore cannot be counted towards the degree at the home institution. For the courses taken abroad to be part of the final university grade, the grades need to be converted into the German grading scheme. Therefore, we require a student who transferred grades, that is count grades he or she achieved at a foreign university towards his or her degree at Goettingen University, to have at least one grade from the foreign university that is within the German grading interval. For students with no grade corresponding to the German grading scheme from courses taken abroad or who did not register their grades from the foreign university, the respective indicator variable is zero.

Dependent Variables. Firstly, we analyze the effect of a sojourn on the final university grade. German university grades range from 1 to 4 with 1 being the best and 4 the worst grade still allowing students to pass. In order to make results internationally comparable, we convert them into the U.S. grading scheme with 4 being the best and 1 the worst grade that is still a pass. ${ }^{21}$

Secondly, we examine whether a study-related visit abroad affects the probability of finishing the bachelor studies in time, i.e. within the standard time period of six semesters. To do so, we create an indicator variable that is equal to one if the student finished within six semesters, and equal to zero if he or she took longer to complete his or her degree.

Independent Variables. We control for the student's pre-university ability by using the grade of the high school leaving certificate. Similar to the university grades, the grades of the high school leaving certificate are converted into the U.S. grading scheme.

To account for the students' socio-economic background, we use, like in Chapter 3 , the type of health insurance a student holds and the purchasing power index

\footnotetext{
${ }^{21}$ We converted the grades into the US grading scale by subtracting the final university grade from five.
} 
related to the zip-code area his or her parents live in. The health insurance status can be used in this context due to the features of the German health care system, distinguishing between private and public health insurance. One has to fulfill certain criteria concerning income or employment status in order to select a private instead of the generally compulsory public health insurance. Therefore, compared to the overall German population, a disproportionately high number of people who hold a high school certificate enabling them to register at a university or a university of applied sciences and people who finished university or university of applied sciences with a degree or even a Ph.D. are privately insured. ${ }^{22}$ Taking into consideration that students in Germany are normally insured through their parents, their health insurance contains information about their socio-economic background.

The purchasing power index is provided by the market research firm GfK and measures the purchasing power within a zip-code area relative to the German average in 2007. ${ }^{23}$ As the German zip-code areas are relatively small - for instance there are about 190 different zip-codes in Berlin - we are confident to apply a well-founded measure of the students' socio-economic background.

We include the distance between the students' home town and Goettingen into our analysis in order to account for prior mobility decisions. ${ }^{24}$ Furthermore, we control for gender, the university's faculties offering bachelor programs and the student's cohort.

Summary Statistics. Summary statistics are shown in Table 4.1. Our final dataset contains 2624 observations, out of which $12 \%$ spent part of their studies at a university in a foreign country. The mean university grade point average (GPA) of these students is slightly higher, i.e. better, than the mean university GPA of their counterparts who stay at home. A t-test shows that this difference is significant at

\footnotetext{
${ }^{22}$ In $2008,56.7 \%$ of the privately insured held a high school degree that enables enrolment at a university or a university of applied sciences, $38.0 \%$ finished university or university of applied sciences with a degree or a Ph.D. (Finkenstädt and Kefler, 2012). The corresponding numbers for the whole German population are $24.4 \%$ and $13.0 \%$ respectively (Statistisches Bundesamt, 2009).

${ }^{23} \mathrm{GfK}$ is one of the biggest companies worldwide in the field of market research. It collects information on people's consumption behavior and lifestyle. The purchasing power index used in the analysis is based on data provided by the German tax offices as well as other relevant statistics concerning e.g. pensions and unemployment benefits.

${ }^{24}$ GoogleMaps standard route planer is used to measure the distance between the parents' zipcode area and Goettingen.
} 
Table 4.1: Summary statistics

\begin{tabular}{lcccccc}
\hline \hline & \multicolumn{2}{c}{ Total } & \multicolumn{2}{c}{ Study } & Abroad $=1$ & \multicolumn{2}{c}{ Study Abroad $=0$} \\
Variable & Mean & Std. Dev. & Mean & Std. Dev. & Mean & Std. Dev. \\
\hline University GPA & 2.86 & 0.47 & 2.99 & 0.44 & 2.85 & 0.47 \\
Graduate in time & 0.59 & 0.49 & 0.36 & 0.48 & 0.62 & 0.48 \\
Study abroad & 0.12 & 0.32 & 1.00 & 0.00 & 0.00 & 0.00 \\
Transfer grades & 0.09 & 0.29 & 0.80 & 0.40 & 0.00 & 0.00 \\
High school GPA & 2.60 & 0.63 & 2.71 & 0.59 & 2.58 & 0.63 \\
Male & 0.46 & 0.50 & 0.36 & 0.48 & 0.48 & 0.50 \\
Private health insurance & 0.18 & 0.39 & 0.23 & 0.42 & 0.18 & 0.38 \\
Purchasing power index & 97.73 & 11.58 & 99.04 & 10.71 & 97.56 & 11.68 \\
Distance to university & 184.69 & 118.41 & 204.25 & 118.91 & 182.13 & 118.13 \\
Medicine & 0.02 & 0.12 & 0.01 & 0.08 & 0.02 & 0.13 \\
Humanities & 0.20 & 0.40 & 0.31 & 0.46 & 0.18 & 0.39 \\
Mathematics & 0.04 & 0.19 & 0.03 & 0.17 & 0.04 & 0.20 \\
Physics & 0.04 & 0.19 & 0.02 & 0.13 & 0.04 & 0.20 \\
Chemistry & 0.05 & 0.21 & 0.01 & 0.10 & 0.05 & 0.22 \\
Geology/Geography & 0.03 & 0.18 & 0.01 & 0.08 & 0.03 & 0.18 \\
Biology & 0.11 & 0.31 & 0.08 & 0.28 & 0.12 & 0.32 \\
Forest sciences & 0.09 & 0.28 & 0.05 & 0.21 & 0.09 & 0.29 \\
Agriculture & 0.21 & 0.41 & 0.17 & 0.37 & 0.22 & 0.41 \\
Economic sciences & 0.18 & 0.38 & 0.27 & 0.44 & 0.17 & 0.37 \\
Social sciences & 0.05 & 0.21 & 0.06 & 0.24 & 0.05 & 0.21 \\
Cohort 1 & 0.08 & 0.27 & 0.04 & 0.20 & 0.08 & 0.28 \\
Cohort 2 & 0.08 & 0.27 & 0.09 & 0.28 & 0.08 & 0.26 \\
Cohort 3 & 0.18 & 0.38 & 0.22 & 0.42 & 0.17 & 0.38 \\
Cohort 4 & 0.35 & 0.48 & 0.36 & 0.48 & 0.35 & 0.48 \\
Cohort 5 & 0.31 & 0.46 & 0.29 & 0.45 & 0.32 & 0.46 \\
\hline Observations & 2624 & & 304 & & 2320 & \\
\hline \hline
\end{tabular}


the $1 \%$ level. The same results hold for the GPA earned at high school.

In our sample, there are slightly more female than male students and it seems that a disproportionately high number of female students go abroad. Students who go on a study-related visit abroad appear to have a higher socio-economic background, accounted for by the private health insurance and the purchasing power index. Moreover, these students seem to be generally more mobile as the mean distance between their parents' home and Goettingen is greater than for students who take all their courses at Goettingen University. These findings are in line with surveys focusing on German students in general (DAAD and HIS, 2013).

Furthermore, it can be seen that $80 \%$ of the students in our sample who go on a study-related visit abroad also count grades from the foreign university towards their degree. When taking a look at the time needed to finish a degree, summary statistics show that only $36 \%$ of the students studying abroad graduate in time, i.e. within six semesters, in comparison to $62 \%$ of their counterparts who stay at home.

The faculties having the highest shares of students studying abroad are the faculty of economic sciences $(27 \%)$ and the faculty of humanities (31\%). For both faculties, this share is disproportionately high compared to their overall share of students (18\% and $20 \%$ respectively) in our sample. The faculty of agriculture, on the other hand, has a disproportionately low share of students who decide to go abroad for part of their studies compared to its overall share of students (17\% and $21 \%$ respectively). These findings are also in line with results found with regard to all German students (DAAD and HIS, 2013).

\subsection{Empirical framework}

The summary statistics suggest that students who go abroad during their university studies might be systematically different from students who stay at home. If this is indeed the case, a direct comparison of the two groups and ordinary least squares regressions may lead to biased results.

The best way to overcome this problem of self-selection into studying abroad would be through the design of an experimental framework, where students are 
randomly assigned to the treatment, i.e. studying abroad. Such a procedure is, however, not feasible for obvious reasons. Since there is no specified threshold at which students become eligible to go abroad also empirical strategies like regression discontinuity designs cannot be applied in our setting. In fact, there exist several different mobility programs and every institute individually allocates the available amount of places on these programs. Thereby, students are not restricted to only applying at the faculty they are studying at. This means that students who want to go abroad have a lot of different possibilities to apply for an international mobility program. Hence, not being accepted for a certain program or at a certain institute does not necessarily imply that the student cannot go abroad at all.

Bearing this in mind, we apply a propensity score matching strategy in order to take self-selection into studying abroad into account as much as possible. The general idea of this matching approach is to compare individuals that have received a certain treatment and individuals of a control group who have not, but who are very similar concerning their pre-treatment characteristics. Since the matched individuals only differ in the treatment, a difference in the outcome can be assigned to the treatment (see for instance Becker and Ichino, 2002; Caliendo and Kopeinig, 2008; Heckman et al., 1998). As it might be difficult to find counterparts that are equal with regard to a variety of covariates, Rosenbaum and Rubin (1983) suggest to use a balancing score in order to group treated and untreated individuals. The balancing score they introduce is the propensity score which measures the conditional probability of being exposed to a treatment given a set of pre-treatment covariates (Becker and Ichino, 2002):

$$
p(X)=\operatorname{Pr}(A=1 \mid X)=E(A \mid X)
$$

where $A$ denotes the treatment, which is studying abroad in our case, and $X$ is a set of pre-treatment covariates.

The treated and untreated individuals are grouped by their propensity scores so that within a respective group, the distribution of covariates is identical and independent of the assignment to the treatment, i.e. receiving the treatment is as 
good as random given the controls. The average treatment effect on the treated, $\tau_{A T T}$, is the difference between the expected outcome when being and not being exposed to the treatment for all individuals who actually received the treatment (Becker and Ichino, 2002):

$$
\tau_{A T T}=E\left[E\left\{Y_{1 i} \mid A_{i}=1, p\left(X_{i}\right)\right\}-E\left\{Y_{0 i} \mid A_{i}=0, p\left(X_{i}\right)\right\} \mid A_{i}=1\right]
$$

with $Y_{1 i}$ and $Y_{0 i}$ being the outcome for individual $i$ in the case that he or she received and did not receive the treatment respectively.

In our analysis we estimate the propensity score of going abroad for all students in the sample using a probit model with $\Phi$ being the standard normal cumulative distribution function and $h\left(X_{i}\right)$ a function of the individuals' covariates:

$$
\operatorname{Pr}\left(A_{i}=1 \mid X_{i}\right)=\Phi\left\{h\left(X_{i}\right)\right\}
$$

From the summary statistics presented in Section 4.2 as well as the results of other studies on the topic (e.g. DAAD and HIS, 2013), we expect the high school leaving grade, gender, socio-economic background, pre-university mobility, field of study and the student's cohort to have an impact on going abroad.

We match treated and untreated individuals based on their propensity scores. Specifically, we first apply nearest neighbor matching. This means that in order to estimate the average treatment effect on the treated, each individual of the treatment group is assigned the counterpart in the control group that is closest with regard to the propensity score. To reduce the risk of bad matches, we require the nearest neighbor to be within a caliper of 0.05 . Additionally, we provide estimation results for kernel matching, with an Epanechnikov kernel function and the standard bandwidth of 0.06 , and radius matching, with a caliper of 0.05 . The letter two algorithms make use of more individuals of the control group at the cost of these additional matches not being as close as the nearest neighbor.

Unconfoundedness and Weak Overlap. A crucial assumption of propensity 
score matching is unconfoundedness or conditional independence: $Y_{0}, Y_{1} \| A \mid X$, with $\|$ denoting independence. This means that given the characteristics we observe, potential outcomes do not depend on treatment assignment. Nonetheless, if there exist unobserved variables which affect both going abroad and success at university, propensity score matching would lead to biased results.

By using the grade of the high school leaving certificate, we control for the fact that students who go abroad might be generally academically more able than students who stay at their home university. The grade of the high school leaving certificate is shown to be a strong predictor for university success (Betts and Morell, 1990; Cyrenne and Chan, 2013; and the results presented in Chapter 3 of this book). Furthermore, the grade may be a measure of motivation since students with a very good high school leaving certificate are typically not only smart, but also willing to put a lot of effort into studying.

The fact that studying abroad might be more costly than staying in Germany may lead to students with a higher socio-economic background being more likely to pursue part of their studies in a foreign country. Going to another country might be difficult to finance, especially for students who cannot afford to move away from their parents' residence when starting university. Moreover, highly educated parents might support a sojourn not only financially, but also by emphasizing the advantages of getting to know another country, language and culture. Therefore, we address a possible self-selection with regard to socio-economic characteristics by using the type of health insurance a student holds and the purchasing power of the parents' zip-code area as controls. Furthermore, we include gender in our model in order to account for systematic differences between male and female students regarding their choice of going abroad as well as academic performance.

Another factor that might influence the decision to go abroad as well as success at university is pre-university mobility. Moving away from home when starting university may imply a high level of independence and openness. Students who already once decided to leave their social environment may be more likely to go to a foreign country than their counterparts who decided to study at a university close to their home town. In addition, pre-university mobility might also affect the final university performance. A possible reason for this could be that students who 
move far away from home when starting university put more effort into finding the perfect match regarding university and field of study. This might lead to a high level of motivation, resulting in better grades. As it seems likely that the impact of pre-university mobility is non-linear with a decreasing marginal effect of distance, we use the natural logarithm of this variable as a control.

We also take into account that the possibility to go abroad as well as university performance may be influenced by the different faculties. Each student is assigned to one of the thirteen faculties at Goettingen university, depending on the field of study. Since a sojourn may be more common and more useful in some fields, such as in foreign language studies, literature and culture, students belonging to those faculties might be more likely to go abroad. At the same time, examination regulations and policies may differ among faculties, and thereby influence the final university grade as well as the time needed to graduate. In Chapter 3, we provide evidence for this assumption.

Finally, we account for cohort effects by controlling for the semester the student started a bachelor program in the field of study he or she obtained his or her degree in. Studying abroad might be more promoted in some years than in others and cohort size as well as the number of available places may differ in different years. Therefore, cohorts could have an impact on the probability of studying at a foreign university. Further, students within the same cohort are affected by the same study regulations and conditions: they may even take the same courses and examinations. As these cohort effects are probably even stronger within each of the faculties, we include interaction effects.

To sum up, we are confident to observe the relevant characteristics that might impact both assignment to the treatment and the outcome variables. Nevertheless, we are aware that propensity score matching only leads to robust and unbiased results if the assumption of conditional independence holds. We address this issue by testing the sensitivity of our results with regard to unobserved heterogeneity in Section 4.5.

Besides unconfoundedness the assumption of weak overlap also needs to be satisfied in order to get robust and unbiased results: $\operatorname{Pr}(A=1 \mid X)<1$. It means that individuals with a given set of covariates have a positive probability of not being 
treated. In our analysis, the weak overlap condition is fulfilled.

\subsection{Results}

We start the empirical analysis with a simple OLS model. Table 4.2 shows a positive and highly significant effect of studying abroad on the university GPA in all three regressions. In the full specification, column (3), the estimated effect of studying abroad is 0.08 grade points, which is slightly larger than the estimated effect of an increase in the high school leaving grade by one fifth of a grade. However, as described above, these results may suffer from a bias due to self-selection.

Table 4.2: Final university grade - OLS results

\begin{tabular}{lccc}
\hline \hline \multicolumn{3}{c}{ Dependent Variable: University GPA } \\
\hline Study abroad & $(1)$ & $(2)$ & $(3)$ \\
& $0.139^{* * *}$ & $0.087^{* * *}$ & $0.084^{* * *}$ \\
High school GPA & $(0.027)$ & $(0.024)$ & $(0.025)$ \\
& & $0.407^{* * *}$ & $0.379^{* * *}$ \\
Male & & $(0.011)$ & $(0.010)$ \\
& & & $0.060^{* * *}$ \\
Private health insurance & & & $(0.014)$ \\
& & & -0.008 \\
Purchasing power index & & & $(0.014)$ \\
& & & $0.003^{* * *}$ \\
Log distance & & & $(0.001)$ \\
& & & 0.014 \\
Constant & & & \\
& $2.848^{* * *}$ & $1.796^{* * *}$ & $1.745^{* * *}$ \\
Faculties included & $(0.011)$ & $(0.028)$ & $(0.088)$ \\
Cohorts included & No & No & Yes \\
Faculties \# cohorts included & No & No & Yes \\
\hline R & No & Yes \\
Observations & 0.009 & 0.309 & 0.477 \\
\hline \hline
\end{tabular}

OLS; coefficients, standard errors in parentheses; clustered by counties; ${ }^{*} p<0.05$, ${ }^{* *} p<0.01,{ }^{* * *} p<0.001$.

The descriptive analysis has already shown that students who study abroad might 
be systematically different from those who stay at their home university. Therefore, we expect that spending some time at a foreign university is not necessarily random and take a closer look to characteristics explaining whether or not a student goes abroad. Table 4.3 presents results of the corresponding probit regression. We display marginal effects for a benchmark student and the coefficients of the underlying regression. The benchmark student is female, publicly insured, studies at the faculty of humanities and belongs to the last cohort of the sample. She is average with regard to all continuous variables.

Our findings confirm the descriptive results of DAAD and HIS (2013) as they show that a better high school grade increases the probability of going abroad. Also, the private health insurance status, which proxies socio-economic background, shows the expected positive sign and is significant. The finding of Parey and Waldinger (2011) is supported in our analysis as we find that earlier mobility decisions have predictive power for studying abroad: The coefficient of the variable measuring distance of the parental address to Goettingen carries a positive sign and is highly significant. Besides, male students are less likely to go abroad.

Based on this regression, we apply propensity score matching as described in Section 4.3. Table 4.A.1 in the appendix to this chapter shows that the matching applied balances the treatment and the control group with respect to all variables used. ${ }^{25}$ In addition, Figures 4.A.1.a and 4.A.1.b present the distributions of students in the two groups by their propensity score before and after nearest neighbor matching.

Table 4.4 contains corresponding matching results. The estimated effect is between 0.095 and 0.17 grades and highly significant. ${ }^{26}$ This corresponds to between one fifth and one third of a standard deviation of university GPA. Our findings confirm the positive effect on the final university grade from studying abroad. The effect in Table 4.4 is larger than the OLS coefficient of studying abroad in the full

\footnotetext{
${ }^{25}$ The balancing table presents results for nearest neighbor matching. However, kernel and radius matching also balance the two groups with respect to all variables used. For the sake of brevity, we leave out corresponding tables.

${ }^{26}$ The statistical inference for the treatment effect does not take into account that the propensity score is estimated. However, repeating the nearest neighbor matching with the teffects psmatch routine of Stata 13 shows that this does not alter our findings. The estimated effect is almost identical and the corresponding Abadie-Imbens standard error is even smaller.
} 
Table 4.3: Probability of studying abroad

\begin{tabular}{|c|c|c|}
\hline \multicolumn{3}{|c|}{ Dependent Variable: Studying Abroad } \\
\hline & $\begin{array}{l}\text { Marginal effects } \\
\text { (1) }\end{array}$ & $\begin{array}{c}\text { Coefficients } \\
\text { (2) }\end{array}$ \\
\hline High school GPA & $\begin{array}{c}0.049^{* * *} \\
(0.012)\end{array}$ & $\begin{array}{c}0.223^{* * *} \\
(0.053)\end{array}$ \\
\hline Male & $\begin{array}{l}-0.030^{*} \\
(0.014)\end{array}$ & $\begin{array}{r}-0.148^{*} \\
(0.069)\end{array}$ \\
\hline Private health insurance & $\begin{array}{l}0.048^{*} \\
(0.021)\end{array}$ & $\begin{array}{l}0.197^{*} \\
(0.080)\end{array}$ \\
\hline Purchasing power index & $\begin{array}{c}0.001 \\
(0.001)\end{array}$ & $\begin{array}{c}0.004 \\
(0.003)\end{array}$ \\
\hline Log distance & $\begin{array}{c}0.030 * * * \\
(0.009)\end{array}$ & $\begin{array}{c}0.135^{* * *} \\
(0.033)\end{array}$ \\
\hline Constant & & $\begin{array}{c}-2.741^{* * *} \\
(0.358)\end{array}$ \\
\hline Faculties included & Yes & Yes \\
\hline Cohorts included & Yes & Yes \\
\hline Faculties \# cohorts included & Yes & Yes \\
\hline Pseudo- $\mathrm{R}^{2}$ & 0.076 & 0.076 \\
\hline Log Likelihood & -842 & -842 \\
\hline Observations & 2411 & 2411 \\
\hline
\end{tabular}

Probit estimation; column (1) marginal effects for benchmark student, column (2) coefficients; standard errors in parentheses; clustered by county; ${ }^{*} p<0.05,{ }^{* *} p<0.01,{ }^{* * *} p<0.001$.

specification in Table 4.2.

Concerning the channels through which studying abroad affects the grade of the degree, at least two main strands of interpretation can be thought of: firstly, an argument based on learning and secondly, an argument based on grades. Spending some time studying at a foreign university allows students to complement their courses at their home institution. They may take specialized courses that are not available at their home university and are potentially confronted with different styles of teaching, learning and studying. In many cases, the language spoken is different. Furthermore, the cultural experience is considered to contribute to the personal development of students. Therefore, going abroad may improve the student's academic ability. 
Table 4.4: Final university grade - matching results

\begin{tabular}{|c|c|c|c|}
\hline \multicolumn{4}{|c|}{ Dependent Variable: University GPA } \\
\hline & \multicolumn{3}{|c|}{ Propensity Score Matching } \\
\hline & $\begin{array}{c}\text { Nearest Neighbor } \\
\text { (1) }\end{array}$ & $\begin{array}{l}\text { Kernel } \\
(2)\end{array}$ & $\begin{array}{c}\text { Radius } \\
(3)\end{array}$ \\
\hline Study abroad & $\begin{array}{c}0.170^{* * *} \\
(0.043)\end{array}$ & $\begin{array}{c}0.095^{* * *} \\
(0.028)\end{array}$ & $\begin{array}{c}0.096^{* * *} \\
(0.028)\end{array}$ \\
\hline Treated observations & 302 & 302 & 302 \\
\hline Untreated observations & 2108 & 2108 & 2108 \\
\hline
\end{tabular}

Propensity score matching, average treatment effects on the treated using nearest neighbor matching with caliper 0.05 (column 1), kernel matching with an Epanechnikov kernel function, bandwidth 0.06 , (column 2), and radius matching with caliper 0.05 (column 3) calculated using PSMATCH2 package for Stata by Leuven, E. and Sianesi, B. (2003) Version 4.0.10; only observations on common support are used; standard errors in parentheses; variables used for the estimation and calculation of the propensity score are high school GPA, male, private health insurance, purchasing power index, log distance to university and indicator variables for faculties and cohorts as well as interactions of faculties and cohorts; ${ }^{*} p<0.05,{ }^{* *} p<0.01,{ }^{* * *} p<0.001$.

The second interpretation refers to grades transferred back to the home university: if these grades are on average better than the average grade earned at the home university, the positive effect shown above can be explained. There are several reasons why this could be the case: For instance, the effect could be based on better teaching or studying conditions at the host university, a more lenient grading policy on average at the foreign universities in the sample or selectively better grades given to visiting students. However, in our opinion, the most convincing reason why grades transferred back are better than the average grade earned at home, is that students primarily count the good grades of the sojourn towards their degree and leave out mediocre ones.

To shed light on the question of how important the grades transferred are for the positive effect on the final university grade shown above, we focus on the subsample of students who studied abroad. Since about $20 \%$ of this group did not transfer grades, we can exploit this variation to analyze the effect of counting grades towards the degree. All students in this subsample studied abroad so that self-selection into going abroad is not an issue. Still, who transfers grades might not be random. However, the data at hand does not support this hypothesis: estimating whether or not students who go abroad transfer grades does not yield any significant effect. Specifically, we do not find evidence that academically stronger students are more likely 
Table 4.5: Transferring grades

\begin{tabular}{lc}
\hline \hline Dependent Variable: & University GPA \\
\hline High school GPA & $0.416^{* * *}$ \\
& $(0.037)$ \\
Transfer grades & $0.213^{* * *}$ \\
& $(0.052)$ \\
Male & 0.082 \\
& $(0.055)$ \\
Private health insurance & 0.046 \\
& $(0.043)$ \\
Purchasing power index & 0.002 \\
& $(0.002)$ \\
Log distance & -0.005 \\
& $(0.024)$ \\
Constant & $1.708^{* * *}$ \\
& $(0.273)$ \\
\hline Faculties included & Yes \\
Cohorts included & Yes \\
Faculties \# cohorts included & Yes \\
\hline $\mathrm{R}^{2}$ & 0.559 \\
Observations & 304 \\
\hline \hline
\end{tabular}

OLS; coefficients, standard errors in parentheses; clustered by county; observations of students who studied abroad; ${ }^{*} p<0.05,{ }^{* *} p<0.01,{ }^{* * *} p<0.001$. 
to transfer grades than weaker students. ${ }^{27}$ Besides, based on theoretical considerations, it is not obvious why some students should systematically be more likely to transfer grades than others. The individual attractiveness of such a transfer should rather depend on how these grades are relative to those that the student earned at the home university.

Table 4.5 presents OLS results for the effect of transferring grades from abroad on the final university grade. The coefficient of interest is highly significant and positive, implying that the grades transferred on average improve the final grade. A descriptive comparison of the individual grades confirms this econometric result: on average, the difference between the grades a student transferred and his or her final university GPA is about 0.2 grades. $^{28}$ The corresponding median difference is even larger, a quarter of a grade.

Our second measure of academic success is whether bachelor students graduate within the standard period of time of six semesters. Table 4.6 presents results of the corresponding probit estimation. According to these estimates, going abroad decreases the probability of graduating in time, whereas a better high school leaving grade increases it. Both effects are significant at $0.1 \%$ level. However, as shown above, the group of students who spent part of their studies abroad is not a random selection. Therefore, we address this issue again by applying propensity score matching. We present results of nearest neighbor, kernel and radius matching in Table 4.7.29 The negative effect of going abroad on the probability of graduating within the standard time period is highly significant and robust with regard to the different matching algorithms. ${ }^{30}$ This suggests that students on average are not simply replacing a semester at their home institution with a semester abroad, but extend their overall time spend at university.

Summarizing our empirical results, we show that spending some time at a foreign university during bachelor studies has a positive effect on the final university

\footnotetext{
${ }^{27}$ Corresponding estimation results are included in the appendix to this chapter in Table 4.A.2.

${ }^{28}$ The corresponding difference in means is significant at the $0.1 \%$ level.

${ }^{29}$ Since the same three matching algorithms as above are applied, additional balancing analysis is not necessary.

${ }^{30}$ We also replicate the nearest neighbor matching with the teffects psmatch routine of Stata 13 employing Abadie-Imbens standard errors. Treatment effect and standard error remain virtually unchanged.
} 
Table 4.6: Graduating in time - probit results

\begin{tabular}{|c|c|c|}
\hline \multicolumn{3}{|c|}{ Dependent Variable: Graduating in Time } \\
\hline & $\begin{array}{c}\text { Marginal effects } \\
\text { (1) }\end{array}$ & $\begin{array}{c}\text { Coefficients } \\
\text { (2) }\end{array}$ \\
\hline Study abroad & $\begin{array}{c}-0.238^{* * *} \\
(0.035)\end{array}$ & $\begin{array}{c}-0.743^{* * *} \\
(0.086)\end{array}$ \\
\hline High school GPA & $\begin{array}{c}0.131^{* * *} \\
(0.021)\end{array}$ & $\begin{array}{c}0.549 * * * \\
(0.053)\end{array}$ \\
\hline Male & $\begin{array}{c}0.018 \\
(0.016)\end{array}$ & $\begin{array}{c}0.076 \\
(0.066)\end{array}$ \\
\hline Private health insurance & $\begin{array}{l}-0.028 \\
(0.021)\end{array}$ & $\begin{array}{l}-0.112 \\
(0.075)\end{array}$ \\
\hline Purchasing power index & $\begin{array}{c}0.001 \\
(0.001)\end{array}$ & $\begin{array}{c}0.004 \\
(0.003)\end{array}$ \\
\hline Log distance & $\begin{array}{c}0.007 \\
(0.005)\end{array}$ & $\begin{array}{c}0.030 \\
(0.021)\end{array}$ \\
\hline Constant & & $\begin{array}{c}-0.949^{* * *} \\
(0.279)\end{array}$ \\
\hline Faculties included & Yes & Yes \\
\hline Cohorts included & Yes & Yes \\
\hline Faculties \# cohorts included & Yes & Yes \\
\hline Pseudo- $\mathrm{R}^{2}$ & 0.223 & 0.223 \\
\hline Log Likelihood & -1361 & -1361 \\
\hline Observations & 2595 & 2595 \\
\hline
\end{tabular}

Probit estimation; column 1: marginal effects for benchmark student; column 2: coefficients, standard errors in parentheses; clustered by county; ${ }^{*} p<0.05,{ }^{* *} p<0.01,{ }^{* * *} p<0.001$. 
Table 4.7: Graduating in time - matching results

\begin{tabular}{lccc}
\hline \hline \multicolumn{4}{c}{ Dependent Variable: Graduating in Time } \\
\hline & \multicolumn{3}{c}{ Propensity Score Matching } \\
\cline { 2 - 4 } & Nearest Neighbor & Kernel & Radius \\
& $(1)$ & $(2)$ & $(3))$ \\
\hline Study abroad & $-0.219^{* * *}$ & $-0.224^{* * *}$ & $-0.226^{* * *}$ \\
& $(0.043)$ & $(0.030)$ & $(0.030)$ \\
\hline Treated observations & 302 & 302 & 302 \\
Untreated observations & 2108 & 2108 & 2108 \\
\hline \hline
\end{tabular}

Propensity score matching, average treatment effects on the treated using nearest neighbor matching with caliper 0.05 (column 1), kernel matching with an Epanechnikov kernel, bandwidth 0.06 , (column 2), and radius matching with caliper 0.05 (column 3) calculated using PSMATCH2 pack(colut 2), a age for Stata by Leuven, E. and Sianesi, B. (2003) Version 4.0.10; only observations on common support are used; standard errors in parentheses; variables used for the estimation and calculation of the propensity score are high school GPA, male, private health insurance, purchasing power index, log distance to university and indicator variables for faculties and cohorts as well as interactions of faculties and cohorts; ${ }^{*} p<0.05,{ }^{* *} p<0.01,{ }^{* * *} p<0.001$.

grade. Taking into account only students who studied abroad, it can be noted that transferring grades significantly improves the bachelor grade. However, a sojourn reduces the probability of finishing a bachelor program within the standard time period. Selective transferring of grades can explain these findings. It seems that students primarily count those grades awarded abroad towards their degree that are better than the average grade they earn at their home institution. Thereby, students can improve the final grade at the cost of extending the time needed to graduate.

\subsection{Sensitivity to unobserved heterogeneity}

As discussed in Section 4.3, propensity score matching is based on the assumption that we observe the relevant pre-treatment characteristics that determine whether a student goes abroad. If there are unobserved factors that influence both treatment assignment and the outcome variables, our estimated effects may be biased. We follow the bounding approach proposed by Rosenbaum (2002) in order to test to what extend our results are sensitive to such unobserved heterogeneity. The idea of this approach is to analyze how much an unobserved variable could cause two individuals with the same pre-treatment characteristics to differ in their odds of 
Table 4.8: Sensitivity analysis

\begin{tabular}{lcc}
\hline \hline$\Gamma$ & $\begin{array}{c}\text { Rosenbaum Bounds } \\
\text { Upper bound significance level } \\
(1)\end{array}$ & $\begin{array}{c}\text { Mantel-Haenszel Bounds } \\
\text { Lower bound significance level } \\
(2)\end{array}$ \\
\hline 1 & $<0.0001$ & $<0.0001$ \\
1.05 & $<0.0001$ & $<0.0001$ \\
1.1 & 0.0001 & $<0.0001$ \\
1.15 & 0.0005 & 0.0002 \\
1.2 & 0.0015 & 0.0004 \\
1.25 & 0.0037 & 0.0009 \\
1.3 & 0.0084 & 0.0020 \\
1.35 & 0.0171 & 0.0039 \\
1.4 & 0.0320 & 0.0071 \\
1.45 & 0.0549 & 0.0123 \\
1.5 & 0.0879 & 0.0201 \\
1.55 & 0.1319 & 0.0314 \\
1.6 & 0.1871 & 0.0468 \\
1.65 & 0.2524 & 0.0670 \\
1.7 & 0.3255 & 0.0924 \\
1.75 & 0.4037 & 0.1234 \\
\hline \hline
\end{tabular}

$\Gamma$ are the odds of differential assignment due to unobserved factors; column (1) calculated using the rbounds Package for Stata by Gangl, M. (2004), Version 1.1.6; column (2) calculated using mhbounds package for Stata by Becker, S. O. and Caliendo, M. (2006), Version 1.1.5.

going abroad without changing the inference of the estimated effects of a sojourn. ${ }^{31}$

Results of the sensitivity analysis for both outcome variables are shown in Table 4.8. Rosenbaum bounds are applied for the final university grade, column (1), and Mantel-Haenszel bounds for the binary outcome, graduating in time, column (2). The values for the variable $\Gamma$ give the differences in the odds of treatment assignment for individuals with the same pre-treatment characteristics that may occur due to unobserved heterogeneity. With regard to the effect of going abroad on the final university grade, we find a positive and significant effect when assuming that there is no hidden bias $(\Gamma=1)$. The effect turns insignificant at a critical value between 1.4 and 1.45. This means that an unobserved variable could cause a difference in the

\footnotetext{
${ }^{31}$ For a detailed description as well as further empirical applications see also Aakvik (2001), Becker and Caliendo (2007), Caliendo et al. (2008), DiPrete and Gangl (2004).
} 
odds of going abroad for two individuals with the same pre-treatment characteristics of more than $40 \%$ without changing the inference of our result. With relation to the impact of studying abroad on the probability of graduating in time, an unobserved bias could even cause the odds of treatment assignment to differ by more than $60 \%$ without turning the effect insignificant. This leads us to the conclusion that the results of our propensity score matching estimation are fairly robust to unobserved heterogeneity.

\subsection{Conclusion}

Using a unique dataset from a German university, this paper empirically investigates the academic gains to expect from a temporary study-related visit to a foreign university. We can apply a propensity score matching strategy due to the variety of individual information in the data. Our results are robust to different matching estimators and unobserved heterogeneity.

The empirical analysis shows that studying abroad on average improves the final university grade achieved at the home institution by $0.095-0.17$ grades. Two possible explanations for this result are that studying abroad improves the students' academic ability or that the grades obtained at the foreign university are better than the average grade achieved at the home institution.

To shed light on this question, we examine the importance of counting grades obtained abroad towards the degree at Goettingen University. We find that students who transfer grades from their study-related visit abroad have a significantly better final university grade than their counterparts who do not count any grade awarded abroad towards their degree. Furthermore, descriptive statistics show that on average the grades a student obtained abroad and transferred towards the degree at Goettingen University is 0.2 grades better than the grade of his or her final university degree. This supports the interpretation that transferring grades is an important channel through which studying abroad affects academic performance.

Finally, it can be seen that a temporary study-related visit abroad decreases the probability of finishing a bachelor program within the standard time period. This finding suggests that students who go abroad do not count enough courses towards 
their degree at their home university as they would need in order to be in time with their studies. Thereby, it strengthens our interpretation that students primarily transfer classes if the grade obtained at the foreign university is better than the average grade they achieved at home.

Through our results, it can be noted that the impact of studying abroad on academic performance is ambiguous. There is a positive effect of a sojourn on the final university grade, but this result seems mainly driven by selective transferring of grades. As a sojourn decreases the probability of graduating in time, the question of gains from studying in a foreign country also depends on the measure of academic performance used.

On the one hand, the shown second dividend can be seen as an unintended consequence of the existing regulations. In this case universities might want to think of alternative arrangements. For instance, students could be required to transfer a certain number of courses or simply to count all courses taken at the foreign university towards their degree at their home university. On the other hand, the positive effect might be seen as a bonus awarded to those students taking the effort of organizing a study-related visit abroad. If policy makers aim to increase the number of students spending some time at a foreign university, they might appreciate this feature.

For students going abroad for one or two semesters the results shown might also be of importance. These students need to decide whether to realize the second dividend of studying at a foreign university, in addition to the expected positive effect on their personality. By selectively transferring grades, they can improve their final grade, but should take into account that this might come at the cost of prolonged studies. 


\section{Appendix to Chapter 4}

Table 4.A.1: Balancing table for nearest neighbor matching

\begin{tabular}{|c|c|c|c|c|c|}
\hline \multirow{2}{*}{ Variable } & \multirow{2}{*}{ Sample } & \multicolumn{2}{|c|}{ Mean } & \multirow{2}{*}{$\overline{\mathrm{Bias}(\%)}$} & \multirow{2}{*}{ t-Statistic } \\
\hline & & Treated & Control & & \\
\hline \multirow{2}{*}{ High school GPA } & Unmatched & 2.71 & 2.57 & 23.9 & $3.81^{* * *}$ \\
\hline & Matched & 2.71 & 2.70 & 1.1 & 0.14 \\
\hline \multirow{2}{*}{ Male } & Unmatched & 0.36 & 0.47 & -21.7 & $-3.48 * * *$ \\
\hline & Matched & 0.36 & 0.42 & -12.2 & -1.50 \\
\hline \multirow{2}{*}{ Private health insurance } & Unmatched & 0.23 & 0.17 & 15.6 & $2.65^{* *}$ \\
\hline & Matched & 0.23 & 0.23 & -0.8 & -0.10 \\
\hline \multirow{2}{*}{ Purchasing power index } & Unmatched & 99.03 & 97.57 & 13.1 & $2.07^{*}$ \\
\hline & Matched & 99.03 & 100.35 & -11.8 & -1.24 \\
\hline \multirow{2}{*}{ Distance to university } & Unmatched & 5.03 & 4.85 & 17.6 & $2.77^{* *}$ \\
\hline & Matched & 5.03 & 5.01 & 2.4 & 0.31 \\
\hline \multirow{2}{*}{ Medicine } & Unmatched & 0.01 & 0.00 & 5.5 & 1.06 \\
\hline & Matched & 0.01 & 0.01 & -4.8 & -0.45 \\
\hline \multirow{2}{*}{ Humanities } & Unmatched & 0.31 & 0.20 & 25.7 & $4.43^{* * *}$ \\
\hline & Matched & 0.31 & 0.27 & 9.2 & 1.07 \\
\hline \multirow{2}{*}{ Mathematics } & Unmatched & 0.03 & 0.03 & -2.5 & -0.40 \\
\hline & Matched & 0.03 & 0.03 & -1.9 & -0.23 \\
\hline \multirow{2}{*}{ Physics } & Unmatched & 0.01 & 0.02 & -4.6 & -0.70 \\
\hline & Matched & 0.01 & 0.02 & -7.9 & -0.91 \\
\hline \multirow{2}{*}{ Chemistry } & Unmatched & 0.01 & 0.05 & -24.7 & $-3.28 * * *$ \\
\hline & Matched & 0.01 & 0.01 & 0.0 & 0.00 \\
\hline \multirow{2}{*}{ Geology/Geography } & Unmatched & 0.01 & 0.01 & -3.7 & -0.56 \\
\hline & Matched & 0.01 & 0.01 & 0.0 & -0.00 \\
\hline \multirow{2}{*}{ Biology } & Unmatched & 0.08 & 0.12 & -11.7 & -1.81 \\
\hline & Matched & 0.08 & 0.07 & 3.3 & 0.46 \\
\hline \multirow{2}{*}{ Forest sciences } & Unmatched & 0.05 & 0.09 & -17.6 & $-2.59 * *$ \\
\hline & Matched & 0.05 & 0.06 & -5.3 & -0.73 \\
\hline \multirow{2}{*}{ Agriculture } & Unmatched & 0.17 & 0.24 & -17.8 & $-2.77^{* *}$ \\
\hline & Matched & 0.17 & 0.15 & 5.8 & 0.78 \\
\hline \multirow{2}{*}{ Economic sciences } & Unmatched & 0.27 & 0.18 & 20.1 & $3.45^{* * *}$ \\
\hline & Matched & 0.26 & 0.31 & -11.9 & -1.35 \\
\hline \multirow{2}{*}{ Social sciences } & Unmatched & 0.06 & 0.05 & 4.0 & 0.67 \\
\hline & Matched & 0.06 & 0.05 & 2.9 & 0.35 \\
\hline \multirow{2}{*}{ Cohort 1} & Unmatched & 0.04 & 0.07 & -13.3 & $-1.98^{*}$ \\
\hline & Matched & 0.04 & 0.04 & 0.0 & -0.00 \\
\hline \multirow{2}{*}{ Cohort 2} & Unmatched & 0.09 & 0.07 & 4.5 & 0.76 \\
\hline & Matched & 0.09 & 0.09 & -2.4 & -0.28 \\
\hline \multirow{2}{*}{ Cohort 3} & Unmatched & 0.22 & 0.17 & 11.5 & 1.94 \\
\hline & Matched & 0.22 & 0.24 & -4.2 & -0.49 \\
\hline Cohort 4 & Unmatched & 0.36 & 0.34 & 4.0 & 0.65 \\
\hline Cohort 4 & Matched & 0.36 & 0.34 & 3.5 & 0.43 \\
\hline & Unmatched & 0.29 & 0.34 & -9.9 & -1.59 \\
\hline Cohort 5 & Matched & 0.29 & 0.29 & 1.4 & 0.18 \\
\hline
\end{tabular}

Treatment: Study abroad; summary statistics for treated and controls before and after matching; interactions between faculties and cohorts included and balanced after matching; column Bias(\%) displays the standardized bias in percent; column t-Statistic shows the statistic of the t-test for equality of means before and after matching; calculated using PSMATCH2 package for Stata by Leuven, E. and Sianesi, B. (2003) Version 4.0.10; ${ }^{*} p<0.05,{ }^{* *} p<0.01,{ }^{* * *} p<0.001$ 
Figure 4.A.1.a: Before matching

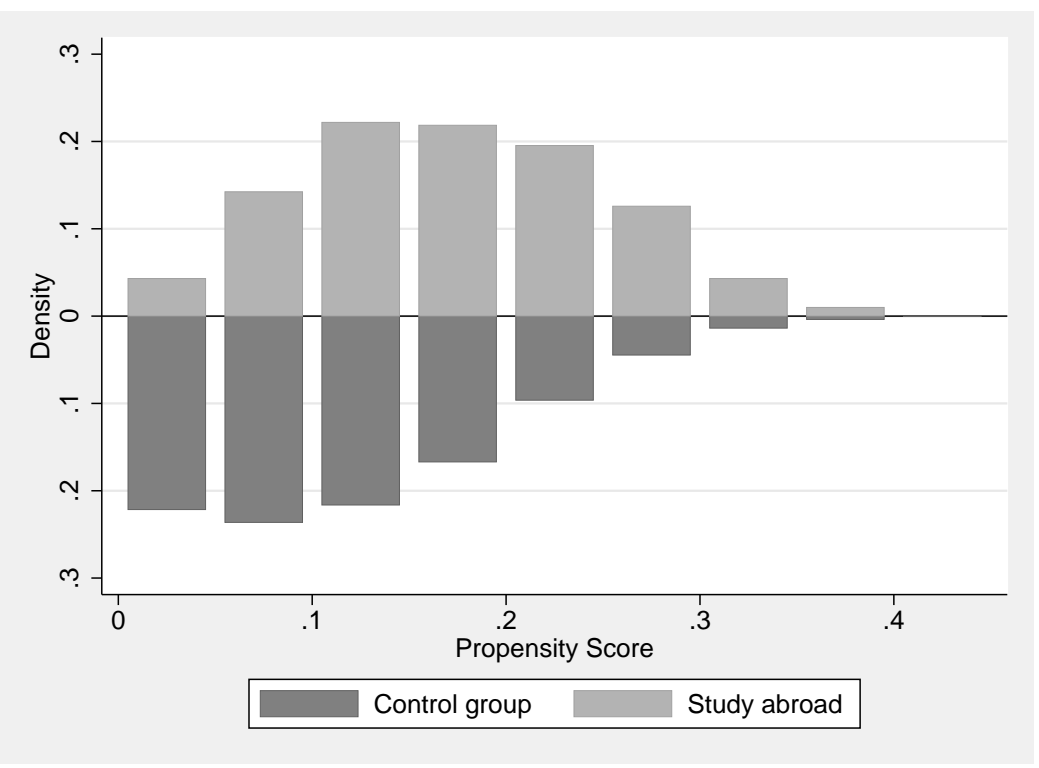

Figure 4.A.1.b: After nearest neighbor matching

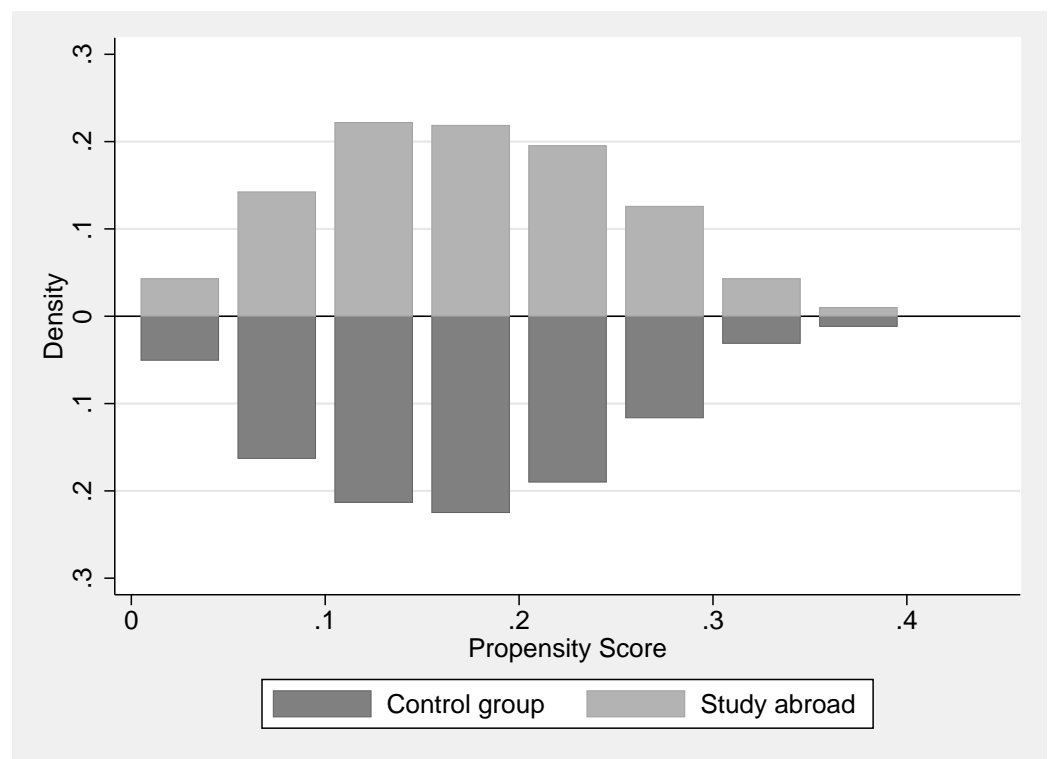

Distribution of the propensity score before and after nearest neighbor matching with caliper 0.05. Calculated using PSMATCH2 package for Stata by Leuven, E. and Sianesi, B. (2003) Version 4.0.10; only observations on common support are used; variables used for the estimation and calculation of the propensity score are high school GPA, male, private health insurance, purchasing power index, log distance to university and indicator variables for faculties and cohorts as well as interactions of faculties and cohorts. 
Table 4.A.2: Determinants of transferring grades

\begin{tabular}{lc}
\hline \hline Dependent Variable: & Transferring Grades \\
\hline High school GPA & Coefficients \\
Male & 0.114 \\
& $(0.167)$ \\
Private health insurance & -0.296 \\
& $(0.202)$ \\
Purchasing power index & 0.060 \\
& $(0.224)$ \\
Log distance & -0.005 \\
& $(0.008)$ \\
Constant & 0.074 \\
& $(0.071)$ \\
\hline Faculties included & 0.397 \\
Cohorts included & $(1.100)$ \\
Faculties \# cohorts included & Yes \\
\hline Pseudo-R & Yes \\
Log Likelihood & Yes \\
Observations & 0.091 \\
\hline \hline
\end{tabular}

Probit estimation; coefficients; standard errors in parentheses; clustered by county; only students who studied abroad, ${ }^{*} p<0.05,{ }^{* *} p<0.01,{ }^{* * *} p<0.001$ 


\section{Chapter 5}

\section{Dynamics of Yardstick Regulation: Historical Cost Data and the Ratchet Effect*}

\subsection{Introduction}

Natural monopolies are frequently subject to regulation. As 'natural' competition does not force prices towards a perfect competition outcome, often regulatory agencies jump in to 'regulate' profit, prices or revenue. Under traditional rate of return regulation, allowed profit of a firm is linked to capital employed. The well known result of Averch and Johnson (1962) is that this regulation provides incentives for the firms to employ an inefficient input mix and not to engage in cost minimizing behavior - in other words, to produce with some slack. Incentive regulation is meant to address this issue. Price cap regulation, originally suggested by Littlechild (1983), decouples costs incurred and prices allowed to be charged by fixing or capping prices, no matter what costs are. Thus, the firm becomes the residual claimant of all costs not incurred and so, has a strong incentive to produce without slack if profit is worth more to the firm than slack is. Necessarily, the question of how the price cap should be defined arises. If the regulator takes into account profits made

*See Meya (2015). 
and costs incurred, the incentive structure is much less clear cut, as e.g. Train (1991) points out. The basic idea of yardstick regulation, as described by Shleifer (1985), solves this problem by using information on costs of other comparable firms to define prices a firm is allowed to charge. In a static world and in every period prices and costs for each individual firm are, as a consequence, completely independent of each other. In the absence of collusion, yardstick regulation fosters efficient production, especially if firms and circumstances of production are very similar. Tangerås $(2002$, p. 232) summarizes: "the regulator is able to extract all surplus from firms and reach full efficiency if technologies are perfectly correlated." This chapter shows that this property does not carry over into a dynamic setting if historical cost data is used. A firm can influence the price it is allowed to charge in the future via its effect on the behavior of other firms. Consequently, without any collusion a ratchet effect can occur under yardstick regulation using historical cost data as a result of individual and independent decision making of firms.

The contribution of this chapter is twofold: In a simple dynamic model with three firms, we show that every firm can affect the price it is allowed to charge if this price is a function of the costs of the other two firms in the period before. By this we highlight a feature of real world applications of yardstick regulation that has not received much attention both in academic literature and in regulatory practice: historical costs are used to define constraints. Furthermore, we compare two variants of yardstick regulation: either the firm with the lowest costs of all firms but the evaluated one, or the average of the other firms can be used as the yardstick. Intuitively, orientation at the best seems to be the tougher form. However, we show that choosing this scheme might lead to higher slack and a worse situation for society.

A well cited example of the use of average performance for regulation is the Prospective Payment System of Medicare (originally Shleifer, 1985), whereas e.g. the German regulation for electricity networks follows a best practice/frontier approach. Yardstick mechanisms are also used in the regulation of, for instance, the water industry in the UK (Cowan, 2006) or railway services in Japan (Mizutani et al., 2009). ${ }^{32}$ Real life examples of yardstick regulation usually have in common that the

\footnotetext{
${ }^{32}$ While this chapter focuses on yardstick regulation of firms, in particular natural monopolies, relative performance measures can be used in a broader range of settings where asymmetric infor-
} 
price of a service offered is set and known before customers use the service. For instance, at the start of a regulatory period prices or constraints are defined based on observations of costs from the regulatory period before.

Aspects of yardstick regulation that are subject to debate or known drawbacks are collusion among firms (e.g. Tangerås, 2002; Potters et al., 2004), investment behavior (e.g. Dalen, 1998; Sobel, 1999) and the potential inability of a regulator to commit to a regulatory scheme for the future (Faure-Grimaud and Reiche, 2005). Moreover, quality might be adversely affected under incentive regulation in general, which makes additional quality regulation necessary (see Sappington, 2005, for a survey). Firms may also lack comparability necessary for the implementation of yardstick regulation (e.g. Laffont and Tirole, 1993). In this chapter, we abstract from these issues and show that the desired outcome, i.e. efficient production, might still not be reached.

We derive our results in a dynamic model with three firms, an infinite horizon and discrete time. As we are interested in the long run effects of the use of historical cost data under yardstick regulation, we focus on the analysis of resulting steady state equilibria. In order to formalize the absence of collusion and Folk Theorem arguments in our result, we define punishment-free Markov-perfect steady state equilibria: these are Markov-perfect steady state Nash equilibria such that firms do not (coordinatedly) choose a uniform slack that is individually optimal for every firm only because of other firms choosing this slack. We show that such equilibria with positive slack, i.e. inefficient production and positive rents for firms, can exist. Furthermore, we show that the highest slack that can exist in such a steady state equilibrium is higher if the firm with the lowest costs of all other firms instead of the average of the other firms is used as the yardstick.

Our modelling of slack, i.e. lack of costly effort, differs from a major part of the contributions to the debate on incentive regulation, represented especially by Laffont and Tirole (1993) or Laffont (1994): in these models costly effort reduces costs of production. In our model, inefficiency costs, slack, are added to real, necessary costs

mation structures are present. For instance, voters may judge incumbent politicians relative to the performance of other politicians in other jurisdictions (Besley and Case, 1995) or workers might be paid based on their ordinal position of performance among their colleagues (Lazear and Rosen, 1981). 
of efficient production and producing with slack offers nonmonetary benefit to the firms. The instantaneous payoff function used is very similar to the one in Blackmon (1994). This is done as it allows for straightforward interpretation of the results and explicitly models the idea that yardstick regulation is meant to solve the inefficiency problem of traditional rate of return regulation. However, this is not a substantive difference but only a different way of presentation.

A key structural element of our model is the time horizon used. In models considering only two periods, the effect driving our results is not present: under yardstick regulation using historical cost data current choices of a firm do not affect the price this firm can charge in the current and the next period. The direct effect is only visible from the next but one period onward. Like Meran and von Hirschhausen (2009) we use dynamic programming techniques to account for long run effects of the decisions of firms. However, we come to differing conclusions. The main difference between their model, which is expanding the model of Shleifer (1985), and our approach leading to these differing results is that Meran and von Hirschhausen (2009) do not allow the firms to benefit from slack and consequently firms do not gain from keeping costs high.

The remainder of this chapter is structured as follows: Section 5.2 explains the model setup. In Section 5.3 all possible punishment-free Markov-perfect steady state equilibria are characterized, existence is proven and the two regulatory schemes are compared with respect to equilibrium outcomes. Section 5.4 concludes.

\subsection{Description of the model}

\subsubsection{Firms}

There are three firms, labelled $j=i, o, x$, each producing a homogeneous output normalized to one. The output is bought by the consumers. For example, one could think of demand for electricity which is very inelastic with respect to price or demand for some crucial medical treatment. These firms could be thought of as catering three comparable regions with electricity grids as local monopolists. The only way they interact in 'competition' is via the regulation imposed on them. In 
every period, the regulator assigns a price to each of the firms. Each firm must not charge more than this price for its output, so the regulator defines a price cap which is equivalent to a revenue cap under the assumption of completely inelastic demand. As demand does not react to price in this setting, all firms always charge the maximum price they are allowed to.

Whereas the firms' output is directly observable the underlying cost structure is unknown to the regulator. Each firm verifiably reports its costs to the regulator who cannot distinguish between 'real' necessary cost, $C>0$, and slack, $S_{t}^{j} \geq 0$, defined as additional costs due to inefficient use of resources, and only observes the sum of both. $C$ does not change over time and is the same for all firms. This is equivalent to assuming that the regulator correctly and completely accounts for all heterogeneity between firms and (exogenous) circumstances of production. ${ }^{33}$ Each firm chooses its slack and may choose different slacks in different periods. For instance, slack can be interpreted as a lack of (costly) effort from managers, oversized offices or all kinds of 'unnecessary' costs that might occur under rate of return regulation. As slack is inefficient production by definition, the regulator maximizing the utility of society desires to avoid all slack without explicit consideration of a target function.

If firm $j$ chooses a positive slack in period $t$, it realizes a nonmonetary utility denoted by $B\left(S_{t}^{j}\right)$. $B$ is twice continuously differentiable with $B(0)=0,1>B^{\prime}>0$ and $B^{\prime \prime}<0$. Accordingly $B\left(S_{t}^{j}\right)<S_{t}^{j}$ for all $S_{t}^{j}>0$. If the sum of necessary costs and slack is smaller than the price the firm is allowed to charge, it additionally realizes a profit. The marginal benefit from an additional unit of profit is constant and normalized to 1 . Increasing profit and decreasing slack are two sides of the same coin as they add up to a constant: the price a firm charges less necessary costs. Hence, it is sufficient to explicitly consider just one of the two as the other one emerges as the residual. The instant payoff function of firm $j$ is in every period given by

$$
F_{t}^{j}=P_{t}^{j}-C-S_{t}^{j}+B\left(S_{t}^{j}\right) .
$$

\footnotetext{
${ }^{33}$ In Shleifer's (1985) one-period model accounting completely and correctly for heterogeneity leads to the efficient equilibrium.
} 
Firms care about profit and slack only. They discount next period's utility with $\delta$, $0<\delta<1$, and maximize their intertemporal utility:

$$
\sum_{t=0}^{\infty} \delta^{t} F_{t}^{j}
$$

Firms need to break even at all times, so that $C+S_{t}^{j} \leq P_{t}^{j}$. Slack is 'expensive' not only from the perspective of the regulator or society: one marginal unit of additional profit always results in higher instantaneous utility for the firm than an additional marginal unit of slack would. The only reason why $S_{t}^{j}>0$ could be an optimal choice of $j$ is that it can affect the price $j$ is allowed to charge in later periods.

We consider an infinite number of periods in order to avoid unrealistic effects of last rounds in which all slack is zero. ${ }^{34}$ Every period there is only one choice per firm to be taken: the slack the firm chooses. The regulatory rule and break even condition are common knowledge, and so are the prices of the current period. Using this knowledge, firms can anticipate how their choice of slack will affect future behavior of the other firms. Accordingly all three current prices are state variables for all $j$.

Strategies are anonymous, so if firms $o$ and $x$ initially do the same, firm $i$ reacts to a change in behavior of $o$ with constant behavior of $x$ just as it would react to a change vice versa. Simple renaming $o$ into $x$ and $x$ into $o$ does not affect the behavior of $i$. Firms simultaneously choose their slack every period without observing the current choice of the other firms.

Only Markov-perfect strategies ${ }^{35}$ are considered, so firms react to the state variables they observe and do not care about the history of states. We exclude collusion between firms as well as arguments based on Folk Theorems, ${ }^{36}$ which can be seen as a form of collusive behavior, from the analysis as yardstick regulation is obviously

\footnotetext{
${ }^{34}$ It is easy to show that a finite horizon and the corresponding backward solution will result in zero slack starting in the very first period.

${ }^{35}$ The corresponding concept of Markov-perfect equilibria goes back to Maskin and Tirole (1988 a and b).

${ }^{36}$ See e.g. Osborne and Rubinstein (1994) for a description of trigger strategies and Folk Theorems.
} 
highly vulnerable to collusion. This chapter does not strive to offer solutions for this issue but proceeds to show that even if all collusive behavior can be avoided, uncoordinated individual utility maximization by firms can result in equilibria with positive slack. Therefore we restrict our attention to strategies that are not based on collusion or coordination and exclude that firms coordinatedly choose a uniform slack that is otherwise not an optimal choice for any individual firm.

\subsubsection{Regulatory rules}

The price a firm is allowed to charge is derived from costs realized by the other two firms in the previous period. We separately look at two regulatory schemes: average yardstick regulation under which average costs of the other firms are used as the yardstick, and frontier yardstick regulation or best practice regulation under which only the costs of the best performing firm of all others, i.e. the firm with the lowest costs, are the yardstick. For example, the price that firm $i$ is allowed to charge in period $t+1$ is accordingly a function of the slack $o$ and $x$ are choosing in $t$ in both cases:

$$
P_{t+1}^{i}=R^{i}\left(S_{t}^{o}, S_{t}^{x}\right)
$$

Under frontier yardstick regulation the price is given by

$$
P_{t+1}^{i}=\min \left(C+S_{t}^{o}, C+S_{t}^{x}\right)=C+\min \left(S_{t}^{o}, S_{t}^{x}\right)
$$

and under average yardstick regulation by

$$
P_{t+1}^{i}=\frac{1}{2} \sum_{j \neq i}\left(C+S_{t}^{j}\right)=C+\frac{1}{2} \sum_{j \neq i} S_{t}^{j} .
$$

Regulatory rules for the other firms and periods are defined analogously. Since necessary costs are constant, $\mathrm{C}$ can be factored out under both regulatory regimes and can be normalized to zero. This is equivalent to interpreting $P_{t+1}^{i}$ as the amount by which the price $i$ may charge in $t+1$ is greater than necessary costs $C{ }^{37}$ In the

\footnotetext{
${ }^{37}$ Necessary costs $C$ remain, however, unknown to the regulator.
} 
first period of yardstick regulation, prices are exogenously given: they could be derived from some regulatory rule that was in place before yardstick regulation was implemented.

Lemma 1. Under both regulatory rules, slacks and prices converge to a steady state in which all firms choose the same slack and realize zero profits due to regulatory mechanics. This slack may be zero.

Proof. See appendix.

As long as not all firms choose the same slack and this slack is equal to the price they are allowed to charge ( $C$ is normalized to zero), the highest slack chosen in $t$ cannot be chosen by any firm anymore in $t+2$ at the latest. Accordingly, there is a downward drift of the highest slack, whenever firms choose differing slacks. As slack cannot become negative, convergence is assured.

\subsection{Equilibrium analysis}

It is easy to show that equilibria with very high slack could exist, given initial prices are sufficiently high, if firms punish other firms' uncooperative behavior. Unilateral punishment conditioned on other firms' past behavior is precluded, by restricting our attention to Markov-perfect strategies. However, firms could follow a Markov-perfect strategy which includes extreme slacks, e.g. zero slack, if they observe a specific vector of prices. From the proof of Lemma 1, it directly follows that under frontier yardstick regulation, every firm can force all firms into a steady state equilibrium with zero slack by choosing zero slack once. This is the worst possible steady state from the perspective of all firms. Therefore, if at least one firm chooses zero slack, all other firms can choose zero slack, and thereby the highest feasible instantaneous profit without adversely affecting future payoffs. Consequently, the best response to other firms choosing an extreme slack could be choosing the same extreme slack. In the spirit of the Folk Theorem (a threat of) 'joint mutual punishment', i.e. firms each choosing an extreme slack because of other firms choosing this slack, could be 
used to implement equilibria with very high slack. Such equilibria would involve aspects of a coordination game.

The analysis of corresponding equilibria does not offer much additional insight as yardstick regulation is known to be highly vulnerable to collusion. Joint mutual punishment, that no firm would do unilaterally, can be seen as a form of collusion. To this end, we explicitly exclude all sorts of joint mutual punishment, collusion or coordination from our analysis and show that steady state equilibria with positive slack that are 'punishment-free' can still exist. Therefore, we restrict our attention to the subset of Markov-perfect equilibria satisfying the following definition: Let $f^{i}\left(\mathbf{P}_{\mathbf{t}}\right), f^{o}\left(\mathbf{P}_{\mathbf{t}}\right)$ and $f^{x}\left(\mathbf{P}_{\mathbf{t}}\right)$ be the Markov-perfect strategies of firms $i, o$ and $x$ respectively, and $\mathbf{P}_{\mathbf{t}}$ be the vector of prices valid for firms $i, o$ and $x$ in period $t$. Consider a Markov-perfect equilibrium $\left(f^{i}\left(\mathbf{P}_{\mathbf{t}}\right), f^{o}\left(\mathbf{P}_{\mathbf{t}}\right), f^{x}\left(\mathbf{P}_{\mathbf{t}}\right)\right)$. It is called punishment-free if for all $\mathbf{P}_{\mathbf{t}}$ where $f^{j}\left(\mathbf{P}_{\mathbf{t}}\right)=f^{k}\left(\mathbf{P}_{\mathbf{t}}\right)$, with $j, k=i, o, x$ and $j \neq k$, at least one strategy $f^{l}\left(\mathbf{P}_{\mathbf{t}}\right)=f^{j}\left(\mathbf{P}_{\mathbf{t}}\right)$, with $l=i, o, x$, is also the best response to all $m=i, o, x$, with $m \neq l$, choosing $S_{t}^{m}>f^{m}\left(\mathbf{P}_{\mathbf{t}}\right)$.

In a punishment-free equilibrium, firms do not choose a uniform slack that is optimal if and only if other firms also choose this slack and that is not an optimal choice of slack for any firm if all other firms choose higher slacks. Thus, whenever firms choose the same slack, for at least one firm, this slack must remain optimal if all but this firm choose higher slacks instead. In other words, we exclude that firms choose a uniform slack that is for each firm optimal only because of other firms doing so.

Below we implicitly define an optimal value of slack each, denoted by $S^{*}$, that maximizes intertemporal utility given current prices under the respective regulatory regime that can characterize a steady state equilibrium. Furthermore, we derive a unique level of slack, $S^{M *}$ under frontier yardstick regulation and $S^{A *}$ under average yardstick regulation, which offers the highest intertemporal utility for the firms under the respective regime and that can exist in a punishment-free Markov-perfect steady state equilibrium, given that prices are sufficiently high. As will be shown, $S^{M *}$ is implicitly defined by

$$
B^{\prime}=1-\delta^{2}
$$


and $S^{A *}$ by

$$
B^{\prime}=1-\frac{\frac{1}{2} \delta^{2}}{1-\frac{1}{2} \delta} .
$$

Equations (5.6) and (5.7) summarize the respective tradeoff between the marginal benefit of reducing slack in the current period and the corresponding marginal costs from adversely affecting future payoff each firm faces every period under both regulatory schemes.

We show that every $S^{*} \epsilon\left[0, S^{M *}\right]$ and $S^{*} \epsilon\left[0, S^{A *}\right]$, under frontier yardstick regulation and average yardstick regulation respectively, can occur in a punishment-free Markov-perfect steady state equilibrium, provided that the initial prices are high enough. Conversely, no other slack is possible in such an equilibrium.

\subsubsection{Optimal slack}

Assume there exists a steady state equilibrium consistent with the triple of punishment-free Markov-perfect strategies of firms $i, o$ and $x$, denoted by $f^{i}\left(\mathbf{P}_{\mathbf{t}}\right), f^{o}\left(\mathbf{P}_{\mathbf{t}}\right)$ and $f^{x}\left(\mathbf{P}_{\mathbf{t}}\right)$ respectively. By definition, strategies need to be optimal in equilibrium. Firms decide on their slack considering their discounted utility in all periods to come given they decide optimally in all future periods given future states. The Principle of Optimality ${ }^{38}$ is used to find the resulting optimal level of slack for firm $i$. So firm $i$ solves the following maximization problem:

$$
J^{i}\left(\mathbf{P}_{\mathbf{t}}\right)=J^{i}\left(P_{t}^{i}, P_{t}^{o}, P_{t}^{x}\right)=\max _{S_{t}^{i} \leq P_{t}^{i}}\left[F\left(P_{t}^{i}, S_{t}^{i}\right)+\delta J^{i}\left(\mathbf{P}_{\mathbf{t}+\mathbf{1}}\right)\right]
$$

where $J^{i}$ denotes the value function of firm $i$ and $\mathbf{P}_{\mathbf{t}+\mathbf{1}}$ is the vector of prices in $t+1$. By Theorem 6.4 and the relaxed Assumption 6.3, i.e. (weak) concavity of the instant payoff function, of Acemoglu (2009) the value function is (weakly) concave in the state variables. Thus, the problem is well-behaved. While the state in $t$ is given, the state in $t+1$ is determined by the regulatory rule. Plugging the general

\footnotetext{
${ }^{38}$ See e.g. Acemoglu (2009) or Stokey, Lucas with Prescott (1989) for a detailed description.
} 
form of this rule in leads to

$$
J^{i}\left(\mathbf{P}_{\mathbf{t}}\right)=\max _{S_{t}^{i} \leq P_{t}^{i}}\left[F\left(P_{t}^{i}, S_{t}^{i}\right)+\delta J^{i}\left(R^{i}\left(S_{t}^{o}, S_{t}^{x}\right), R^{o}\left(S_{t}^{i}, S_{t}^{x}\right), R^{x}\left(S_{t}^{i}, S_{t}^{o}\right)\right)\right]
$$

Just as firm $i$, firms $o$ and $x$ maximize their intertemporal utility given the state variables they observe. So

$$
S_{t}^{o}=f^{o}\left(\mathbf{P}_{\mathbf{t}}\right)
$$

and

$$
S_{t}^{x}=f^{x}\left(\mathbf{P}_{\mathbf{t}}\right)
$$

describe the optimal slack of $o$ and $x$ given $\mathbf{P}_{\mathbf{t}}$, i.e. $S_{t}^{o}$ and $S_{t}^{x}$ satisfy the respective versions of (5.9).

Assuming that $o$ and $x$ follow $f^{o}\left(\mathbf{P}_{\mathbf{t}}\right)$ and $f^{x}\left(\mathbf{P}_{\mathbf{t}}\right)$, respectively, we obtain with (5.9)

$$
\begin{aligned}
J^{i}\left(\mathbf{P}_{\mathbf{t}}\right)=\max _{S_{t}^{i} \leq P_{t}^{i}}\left[F\left(P_{t}^{i}, S_{t}^{i}\right)\right. \\
\left.\quad+\delta J^{i}\left(R^{i}\left(f^{o}\left(\mathbf{P}_{\mathbf{t}}\right), f^{x}\left(\mathbf{P}_{\mathbf{t}}\right)\right), R^{o}\left(S_{t}^{i}, f^{x}\left(\mathbf{P}_{\mathbf{t}}\right)\right), R^{x}\left(S_{t}^{i}, f^{o}\left(\mathbf{P}_{\mathbf{t}}\right)\right)\right)\right] .
\end{aligned}
$$

As this is a constrained maximization problem, we rewrite (5.12) as

$$
\begin{aligned}
J^{i}\left(\mathbf{P}_{\mathbf{t}}\right)=\max _{S_{t}^{i}}[ & F\left(P_{t}^{i}, S_{t}^{i}\right) \\
& +\delta J^{i}\left(R^{i}\left(f^{o}\left(\mathbf{P}_{\mathbf{t}}\right), f^{x}\left(\mathbf{P}_{\mathbf{t}}\right)\right), R^{o}\left(S_{t}^{i}, f^{x}\left(\mathbf{P}_{\mathbf{t}}\right)\right), R^{x}\left(S_{t}^{i}, f^{o}\left(\mathbf{P}_{\mathbf{t}}\right)\right)\right) \\
& \left.+\lambda_{t}^{i}\left(P_{t}^{i}-S_{t}^{i}\right)\right]
\end{aligned}
$$

with the complementary slackness conditions

$$
\lambda_{t}^{i} \geq 0 \text { and } \lambda_{t}^{i}\left(P_{t}^{i}-S_{t}^{i}\right)=0 .
$$

The corresponding first order condition (FOC) for the maximum problem is given 
by:

$$
F_{2}\left(P_{t}^{i}, S_{t}^{i}\right)+\delta J_{2}^{i}\left(\mathbf{P}_{\mathbf{t}+\mathbf{1}}\right) \cdot R_{1}^{o}\left(S_{t}^{i}, S_{t}^{x}\right)+\delta J_{3}^{i}\left(\mathbf{P}_{\mathbf{t}+\mathbf{1}}\right) \cdot R_{1}^{x}\left(S_{t}^{i}, S_{t}^{o}\right)-\lambda_{t}^{i}=0 .
$$

Accordingly numbers as the lower index mark derivatives and the number describes the argument with respect to which the derivative is taken. The upper index describes the function from which the derivative is taken. If the lower index includes a ' $t$ ', it is a time index. So, $R_{1}^{o}\left(S_{t}^{i}, S_{t}^{x}\right)$ describes how the price $o$ may charge in $t+1$ reacts to a marginal change of the slack of $i$ in $t$. We only need to look at derivatives to the left, i.e. reductions of slack, as starting from a steady state no firm can increase its slack without violating the break even constraint. Accordingly, throughout this chapter, all derivatives are to be understood as left hand side derivatives, i.e. reductions of the respective variable. The corresponding derivatives of the regulatory rules are given in the appendix to this chapter.

Now let

$$
S_{t}^{i}=f^{i}\left(\mathbf{P}_{\mathbf{t}}\right)
$$

describe the optimal slack of firm $i$ given $\mathbf{P}_{\mathbf{t}}$, i.e. $f^{i}\left(\mathbf{P}_{\mathbf{t}}\right)$ is the solution to (5.15). Inserting this into (5.13) leads to:

$$
\begin{aligned}
J^{i}\left(\mathbf{P}_{\mathbf{t}}\right)= & F\left(P_{t}^{i}, f^{i}\left(\mathbf{P}_{\mathbf{t}}\right)\right) \\
& +\delta J^{i}\left(R^{i}\left(f^{o}\left(\mathbf{P}_{\mathbf{t}}\right), f^{x}\left(\mathbf{P}_{\mathbf{t}}\right)\right), R^{o}\left(f^{i}\left(\mathbf{P}_{\mathbf{t}}\right), f^{x}\left(\mathbf{P}_{\mathbf{t}}\right)\right), R^{x}\left(f^{i}\left(\mathbf{P}_{\mathbf{t}}\right), f^{o}\left(\mathbf{P}_{\mathbf{t}}\right)\right)\right) \\
& +\lambda_{t}^{i}\left(P_{t}^{i}-f^{i}\left(\mathbf{P}_{\mathbf{t}}\right)\right) .
\end{aligned}
$$

Taking the derivative to the left with respect $P_{t}^{i}$ we find with Envelope Theorem:

$$
\begin{aligned}
J_{1}^{i}\left(\mathbf{P}_{\mathbf{t}}\right)= & F_{1}\left(P_{t}^{i}, S_{t}^{i}\right) \\
& +\delta J_{1}^{i}\left(\mathbf{P}_{\mathbf{t}+\mathbf{1}}\right) \cdot R_{1}^{i}\left(S_{t}^{o}, S_{t}^{x}\right) \cdot f_{1}^{o}\left(\mathbf{P}_{\mathbf{t}}\right)+\delta J_{1}^{i}\left(\mathbf{P}_{\mathbf{t}+\mathbf{1}}\right) \cdot R_{2}^{i}\left(S_{t}^{o}, S_{t}^{x}\right) \cdot f_{1}^{x}\left(\mathbf{P}_{\mathbf{t}}\right) \\
& +\delta J_{2}^{i}\left(\mathbf{P}_{\mathbf{t}+\mathbf{1}}\right) \cdot R_{2}^{o}\left(S_{t}^{i}, S_{t}^{x}\right) \cdot f_{1}^{x}\left(\mathbf{P}_{\mathbf{t}}\right)+\delta J_{3}^{i}\left(\mathbf{P}_{\mathbf{t}+\mathbf{1}}\right) \cdot R_{2}^{x}\left(S_{t}^{i}, S_{t}^{o}\right) \cdot f_{1}^{o}\left(\mathbf{P}_{\mathbf{t}}\right) \\
& +\lambda_{t}^{i} .
\end{aligned}
$$


Analogously we find

$$
\begin{aligned}
J_{2}^{i}\left(\mathbf{P}_{\mathbf{t}}\right)= & \delta J_{1}^{i}\left(\mathbf{P}_{\mathbf{t}+\mathbf{1}}\right) \cdot R_{1}^{i}\left(S_{t}^{o}, S_{t}^{x}\right) \cdot f_{2}^{o}\left(\mathbf{P}_{\mathbf{t}}\right)+\delta J_{1}^{i}\left(\mathbf{P}_{\mathbf{t}+\mathbf{1}}\right) \cdot R_{2}^{i}\left(S_{t}^{o}, S_{t}^{x}\right) \cdot f_{2}^{x}\left(\mathbf{P}_{\mathbf{t}}\right) \\
+ & \delta J_{2}^{i}\left(\mathbf{P}_{\mathbf{t}+\mathbf{1}}\right) \cdot R_{2}^{o}\left(S_{t}^{i}, S_{t}^{x}\right) \cdot f_{2}^{x}\left(\mathbf{P}_{\mathbf{t}}\right)+\delta J_{3}^{i}\left(\mathbf{P}_{\mathbf{t}+\mathbf{1}}\right) \cdot R_{2}^{x}\left(S_{t}^{i}, S_{t}^{o}\right) \cdot f_{2}^{o}\left(\mathbf{P}_{\mathbf{t}}\right)
\end{aligned}
$$

and

$$
\begin{aligned}
J_{3}^{i}\left(\mathbf{P}_{\mathbf{t}}\right)= & \delta J_{1}^{i}\left(\mathbf{P}_{\mathbf{t}+\mathbf{1}}\right) \cdot R_{1}^{i}\left(S_{t}^{o}, S_{t}^{x}\right) \cdot f_{3}^{o}\left(\mathbf{P}_{\mathbf{t}}\right)+\delta J_{1}^{i}\left(\mathbf{P}_{\mathbf{t}+\mathbf{1}}\right) \cdot R_{2}^{i}\left(S_{t}^{o}, S_{t}^{x}\right) \cdot f_{3}^{x}\left(\mathbf{P}_{\mathbf{t}}\right) \\
+ & \delta J_{2}^{i}\left(\mathbf{P}_{\mathbf{t}+\mathbf{1}}\right) \cdot R_{2}^{o}\left(S_{t}^{i}, S_{t}^{x}\right) \cdot f_{3}^{x}\left(\mathbf{P}_{\mathbf{t}}\right)+\delta J_{3}^{i}\left(\mathbf{P}_{\mathbf{t}+\mathbf{1}}\right) \cdot R_{2}^{x}\left(S_{t}^{i}, S_{t}^{o}\right) \cdot f_{3}^{o}\left(\mathbf{P}_{\mathbf{t}}\right) .
\end{aligned}
$$

Updating (5.19) and (5.20) by one period yields

$$
\begin{aligned}
J_{2}^{i}\left(\mathbf{P}_{\mathbf{t}+\mathbf{1}}\right)= & \delta J_{1}^{i}\left(\mathbf{P}_{\mathbf{t}+\mathbf{2}}\right) \cdot R_{1}^{i}\left(S_{t+1}^{o}, S_{t+1}^{x}\right) \cdot f_{2}^{o}\left(\mathbf{P}_{\mathbf{t}+\mathbf{1}}\right) \\
& +\delta J_{1}^{i}\left(\mathbf{P}_{\mathbf{t}+\mathbf{2}}\right) \cdot R_{2}^{i}\left(S_{t+1}^{o}, S_{t+1}^{x}\right) \cdot f_{2}^{x}\left(\mathbf{P}_{\mathbf{t}+\mathbf{1}}\right) \\
& +\delta J_{2}^{i}\left(\mathbf{P}_{\mathbf{t}+\mathbf{2}}\right) \cdot R_{2}^{o}\left(S_{t+1}^{i}, S_{t+1}^{x}\right) \cdot f_{2}^{x}\left(\mathbf{P}_{\mathbf{t}+\mathbf{1}}\right) \\
& +\delta J_{3}^{i}\left(\mathbf{P}_{\mathbf{t}+\mathbf{2}}\right) \cdot R_{2}^{x}\left(S_{t+1}^{i}, S_{t+1}^{o}\right) \cdot f_{2}^{o}\left(\mathbf{P}_{\mathbf{t}+\mathbf{1}}\right)
\end{aligned}
$$

and

$$
\begin{aligned}
J_{3}^{i}\left(\mathbf{P}_{\mathbf{t}+\mathbf{1}}\right)= & \delta J_{1}^{i}\left(\mathbf{P}_{\mathbf{t}+\mathbf{2}}\right) \cdot R_{1}^{i}\left(S_{t+1}^{o}, S_{t+1}^{x}\right) \cdot f_{3}^{o}\left(\mathbf{P}_{\mathbf{t}+\mathbf{1}}\right) \\
& +\delta J_{1}^{i}\left(\mathbf{P}_{\mathbf{t}+\mathbf{2}}\right) \cdot R_{2}^{i}\left(S_{t+1}^{o}, S_{t+1}^{x}\right) \cdot f_{3}^{x}\left(\mathbf{P}_{\mathbf{t}+\mathbf{1}}\right) \\
& +\delta J_{2}^{i}\left(\mathbf{P}_{\mathbf{t}+\mathbf{2}}\right) \cdot R_{2}^{o}\left(S_{t+1}^{i}, S_{t+1}^{x}\right) \cdot f_{3}^{x}\left(\mathbf{P}_{\mathbf{t}+\mathbf{1}}\right) \\
& +\delta J_{3}^{i}\left(\mathbf{P}_{\mathbf{t}+\mathbf{2}}\right) \cdot R_{2}^{x}\left(S_{t+1}^{i}, S_{t+1}^{o}\right) \cdot f_{3}^{o}\left(\mathbf{P}_{\mathbf{t}+\mathbf{1}}\right) .
\end{aligned}
$$


Plugging (5.21) and (5.22) into the FOC (5.15) leads to

$$
\begin{aligned}
0=F_{2}\left(P_{t}^{i}, S_{t}^{i}\right) & \\
+\delta R_{1}^{o}\left(S_{t}^{i}, S_{t}^{x}\right) \cdot & \left(\delta J_{1}^{i}\left(\mathbf{P}_{\mathbf{t}+\mathbf{2}}\right) \cdot R_{1}^{i}\left(S_{t+1}^{o}, S_{t+1}^{x}\right) \cdot f_{2}^{o}\left(\mathbf{P}_{\mathbf{t}+\mathbf{1}}\right)\right. \\
& +\delta J_{1}^{i}\left(\mathbf{P}_{\mathbf{t}+\mathbf{2}}\right) \cdot R_{2}^{i}\left(S_{t+1}^{o}, S_{t+1}^{x}\right) \cdot f_{2}^{x}\left(\mathbf{P}_{\mathbf{t}+\mathbf{1}}\right) \\
& +\delta J_{2}^{i}\left(\mathbf{P}_{\mathbf{t}+\mathbf{2}}\right) \cdot R_{2}^{o}\left(S_{t+1}^{i}, S_{t+1}^{x}\right) \cdot f_{2}^{x}\left(\mathbf{P}_{\mathbf{t}+\mathbf{1}}\right) \\
& \left.+\delta J_{3}^{i}\left(\mathbf{P}_{\mathbf{t}+\mathbf{2}}\right) \cdot R_{2}^{x}\left(S_{t+1}^{i}, S_{t+1}^{o}\right) \cdot f_{2}^{o}\left(\mathbf{P}_{\mathbf{t}+\mathbf{1}}\right)\right) \\
+\delta R_{1}^{x}\left(S_{t}^{i}, S_{t}^{o}\right) \cdot & \left(\delta J_{1}^{i}\left(\mathbf{P}_{\mathbf{t}+\mathbf{2}}\right) \cdot R_{1}^{i}\left(S_{t+1}^{o}, S_{t+1}^{x}\right) \cdot f_{3}^{o}\left(\mathbf{P}_{\mathbf{t}+\mathbf{1}}\right)\right. \\
& +\delta J_{1}^{i}\left(\mathbf{P}_{\mathbf{t + 2}}\right) \cdot R_{2}^{i}\left(S_{t+1}^{o}, S_{t+1}^{x}\right) \cdot f_{3}^{x}\left(\mathbf{P}_{\mathbf{t}+\mathbf{1}}\right) \\
& +\delta J_{2}^{i}\left(\mathbf{P}_{\mathbf{t + 2}}\right) \cdot R_{2}^{o}\left(S_{t+1}^{i}, S_{t+1}^{x}\right) \cdot f_{3}^{x}\left(\mathbf{P}_{\mathbf{t}+\mathbf{1}}\right) \\
& \left.+\delta J_{3}^{i}\left(\mathbf{P}_{\mathbf{t + 2}}\right) \cdot R_{2}^{x}\left(S_{t+1}^{i}, S_{t+1}^{o}\right) \cdot f_{3}^{o}\left(\mathbf{P}_{\mathbf{t}+\mathbf{1}}\right)\right) \\
-\lambda_{t}^{i} . &
\end{aligned}
$$

In equation (5.23), we clearly see the consequence of the use of historical cost data under yardstick regulation: The price that firm $i$ can charge in the future is influenced by its behavior today. The choice of slack of $i$ in $t$ does not only define its instantaneous payoff, implicitly represented by $F_{2}\left(P_{t}^{i}, S_{t}^{i}\right)$, but also affects the prices $o$ and $x$ can charge in $t+1$ via the regulatory rule, $R^{o}\left(S_{t}^{i}, S_{t}^{x}\right)$ and $R^{x}\left(S_{t}^{i}, S_{t}^{o}\right)$ respectively. Firms $o$ and $x$ choose their slack in $t+1$ based on the state they observe and under the restriction that they have to break even according to their strategies, $f^{o}\left(\mathbf{P}_{\mathbf{t}+\mathbf{1}}\right)$ and $f^{x}\left(\mathbf{P}_{\mathbf{t}+\mathbf{1}}\right)$. The slacks $o$ and $x$ choose in $t+1$, via the regulatory rule, then affect $P_{t+2}^{o}$ and $P_{t+2}^{x}$ and determine the price $i$ is allowed to charge in $t+2$, $P_{t+2}^{i}$. These three prices are the arguments of the value function of $i$ and in period $t$, firm $i$ discounts the effects in $t+2$ with $\delta^{2}$.

From Lemma 1, we know that in every steady state all firms choose the same slack. Thus, starting from a steady state unilateral reduction of the slack of $i$ affects the price $o$ and $x$ may charge in the following period the same way so that 
$R_{1}^{o}\left(S_{t}^{i}, S_{t}^{x}\right)=R_{1}^{x}\left(S_{t}^{i}, S_{t}^{o}\right){ }^{39}$ This reduces $(5.23)$ to

$$
\begin{aligned}
0=F_{2}\left(P_{t}^{i}, S_{t}^{i}\right) & \\
+\delta R_{1}^{o}\left(S_{t}^{i}, S_{t}^{x}\right) & \cdot\left(\delta J_{1}^{i}\left(\mathbf{P}_{\mathbf{t + 2}}\right) \cdot R_{1}^{i}\left(S_{t+1}^{o}, S_{t+1}^{x}\right) \cdot\left[f_{2}^{o}\left(\mathbf{P}_{\mathbf{t}+\mathbf{1}}\right)+f_{3}^{o}\left(\mathbf{P}_{\mathbf{t}+\mathbf{1}}\right)\right]\right. \\
& +\delta J_{1}^{i}\left(\mathbf{P}_{\mathbf{t}+\mathbf{2}}\right) \cdot R_{2}^{i}\left(S_{t+1}^{o}, S_{t+1}^{x}\right) \cdot\left[f_{2}^{x}\left(\mathbf{P}_{\mathbf{t}+\mathbf{1}}\right)+f_{3}^{x}\left(\mathbf{P}_{\mathbf{t}+\mathbf{1}}\right)\right] \\
& +\delta J_{2}^{i}\left(\mathbf{P}_{\mathbf{t + 2}}\right) \cdot R_{2}^{o}\left(S_{t+1}^{i}, S_{t+1}^{x}\right) \cdot\left[f_{2}^{x}\left(\mathbf{P}_{\mathbf{t}+\mathbf{1}}\right)+f_{3}^{x}\left(\mathbf{P}_{\mathbf{t + 1}}\right)\right] \\
& \left.+\delta J_{3}^{i}\left(\mathbf{P}_{\mathbf{t + 2}}\right) \cdot R_{2}^{x}\left(S_{t+1}^{i}, S_{t+1}^{o}\right) \cdot\left[f_{2}^{o}\left(\mathbf{P}_{\mathbf{t}+\mathbf{1}}\right)+f_{3}^{o}\left(\mathbf{P}_{\mathbf{t + 1}}\right)\right]\right)
\end{aligned}
$$

$-\lambda_{t}^{i}$

From Lemma 1 it also follows that, due to regulatory mechanics, in all steady states all firms realize zero profits, i.e. all firms choose the slack that is equal to the maximum price that each firm may charge. So, starting from a steady state a marginal unilateral reduction of the slack of $i$ in $t$ leads to $P_{t+1}^{o}=P_{t+1}^{x}<P_{t+1}^{i}$. Following a punishment-free strategy, the two other firms, $o$ and $x$, will under both regulatory schemes reduce their slack the next period by exactly the resulting marginal reduction of their respective price, given the price they face is not higher than the unique optimal slack $S^{M *}$ and $S^{A *}$, respectively. We formalize this in the following Lemma, considering reductions of slack only for both regulatory regimes:

\section{Lemma 2.}

(i) Frontier yardstick regulation:

If $P_{t+1}^{o}=P_{t+1}^{x} \leq P_{t+1}^{i}$ and $P_{t+1}^{o}=P_{t+1}^{x} \leq S^{M *}$,

then $f_{2}^{o}\left(\mathbf{P}_{\mathbf{t}+\mathbf{1}}\right)+f_{3}^{o}\left(\mathbf{P}_{\mathbf{t}+\mathbf{1}}\right)=f_{2}^{x}\left(\mathbf{P}_{\mathbf{t}+\mathbf{1}}\right)+f_{3}^{x}\left(\mathbf{P}_{\mathbf{t}+\mathbf{1}}\right)=1$.

(ii) Average yardstick regulation:

If $P_{t+1}^{o}=P_{t+1}^{x} \leq P_{t+1}^{i}$ and $P_{t+1}^{o}=P_{t+1}^{x} \leq S^{A *}$, then $f_{2}^{o}\left(\mathbf{P}_{\mathbf{t}+\mathbf{1}}\right)+f_{3}^{o}\left(\mathbf{P}_{\mathbf{t}+\mathbf{1}}\right)=f_{2}^{x}\left(\mathbf{P}_{\mathbf{t}+\mathbf{1}}\right)+f_{3}^{x}\left(\mathbf{P}_{\mathbf{t}+\mathbf{1}}\right)=1$.

\section{Proof. See appendix.}

Intuitively, Lemma 2 means the following: Starting from a steady state, a firm has to reduce its slack if the price that this firm can charge is reduced as it needs to break even. Given that the firm would not voluntarily unilaterally deviate from

\footnotetext{
${ }^{39}$ We extensively deal with the derivatives of the regulatory rules in the appendix to this chapter.
} 
the steady state equilibrium, it cannot increase its intertemporal payoff by deviating even more than necessary. The fact that another firm also has to reduce its slack by the same amount does not cause additional effects in this case.

With Lemma 2, equation (5.24) reduces to

$$
\begin{aligned}
0= & F_{2}\left(P_{t}^{i}, S_{t}^{i}\right) \\
& +\delta R_{1}^{o}\left(S_{t}^{i}, S_{t}^{x}\right) \\
& \cdot\left(\delta J_{1}^{i}\left(\mathbf{P}_{\mathbf{t + 2}}\right) \cdot\left[R_{1}^{i}\left(S_{t+1}^{o}, S_{t+1}^{x}\right)+R_{2}^{i}\left(S_{t+1}^{o}, S_{t+1}^{x}\right)\right]\right. \\
& +\delta J_{2}^{i}\left(\mathbf{P}_{\mathbf{t + 2}}\right) \cdot R_{2}^{o}\left(S_{t+1}^{i}, S_{t+1}^{x}\right) \\
& \left.+\delta J_{3}^{i}\left(\mathbf{P}_{\mathbf{t + 2}}\right) \cdot R_{2}^{x}\left(S_{t+1}^{i}, S_{t+1}^{o}\right)\right) \\
& -\lambda_{t}^{i}
\end{aligned}
$$

To show how the solutions to this equation differ under both regulatory schemes we need to look at them separately.

Frontier yardstick regulation. From Lemma 1 it followed that in all steady state equilibria firms choose the same slack and the slack is equal to each firm's price due to regulatory mechanics. Therefore, in such a steady state $i$ will choose the same slack every period, i.e. $S_{t+1}^{i}=S_{t}^{i}=S^{*}$. Every period $i$ could deviate by reducing its slack. ${ }^{40}$ So, $S^{*}$ must solve the FOC in every period. Now assume $i$ marginally reduces its slack in $t$. From the FOC, it directly follows that it cannot be optimal for $i$ to choose a higher slack in $t+1$ than in $t$. With $S_{t}^{i}<S_{t+1}^{i}$, the slacks of $o$ and $x$ would have to be smaller than the one $i$ chooses in $t+1$ from the regulatory rule and the break even constraint. Accordingly, in $t+1$ the left hand side derivatives of the regulatory rule with respect to the slack of $i$ drop to zero if $S_{t}^{i}<S_{t+1}^{i}$. It follows that $S_{t}^{i}<S_{t+1}^{i}$ cannot describe an optimal strategy of $i$ : the FOC would not hold in $t+1$ as $F_{2}\left(P_{t}^{i}, S_{t}^{i}\right)=B^{\prime}-1$ is smaller than zero and $\lambda_{t+1}^{i}$ is nonnegative from the complementary slackness conditions. We conclude that $i$ marginally reduces its slack in periods $t$ and $t+1$, so that $S_{t}^{i}=S_{t+1}^{i}<S_{t}^{o}=S_{t}^{x}$. From the regulatory rule, equation (5.4), the prices $o$ and $x$ may charge in $t+1$ decrease to $P_{t+1}^{o}=P_{t+1}^{x}=S_{t}^{i}$ and given $S_{t}^{i}=S_{t+1}^{i}$, there is no additional effect on $P_{t+2}^{o}=P_{t+2}^{x}$ from the forced change in the behavior of $o$ and $x$ in $t+1$ : The prices $o$ and $x$ may charge in $t+2$ are

\footnotetext{
${ }^{40}$ No firm can increase its slack in a steady state because of the break even constraint.
} 
given by $P_{t+2}^{o}=\min \left(S_{t+1}^{i}, S_{t+1}^{x}\right)$ and $P_{t+2}^{x}=\min \left(S_{t+1}^{i}, S_{t+1}^{o}\right)$. So, if $o$ and $x$ decrease their slack in $t+1$ to $S_{t}^{i}=S_{t+1}^{i}$, they neither change $P_{t+2}^{o}$ nor $P_{t+2}^{x}$. Consequently, in this situation the left hand side derivatives of the regulatory rule are given by $R_{2}^{o}\left(S_{t+1}^{i}, S_{t+1}^{x}\right)=R_{2}^{x}\left(S_{t+1}^{i}, S_{t+1}^{o}\right)=0 .^{41}$

Intuitively, $i$ decides about its slack in $t$, knowing that its slack in $t+1$ will be the same as in $t$. Hence, deciding about slack in $t$ and $t+1$, firm $i$ knows that $P_{t+2}^{o}$ and $P_{t+2}^{x}$ are equal to $S_{t+1}^{i}$ for all $S_{t+1}^{x} \geq S_{t+1}^{i}$ and $S_{t+1}^{o} \geq S_{t+1}^{i}$ respectively. Accordingly, the only price in $t+2$ that is changed as a consequence of the induced reduction of the slack of $o$ and $x$ to $S_{t+1}^{o}=S_{t+1}^{x}=S_{t+1}^{i}=S_{t}^{i}$ is the price that firm $i$ itself can charge in $t+2, P_{t+2}^{i}$. Further, $R_{1}^{i}\left(S_{t+1}^{o}, S_{t+1}^{x}\right)+R_{2}^{i}\left(S_{t+1}^{o}, S_{t+1}^{x}\right)=1$ is always true under frontier yardstick regulation (see appendix) and therefore equation (5.25) reduces to

$$
0=F_{2}\left(P_{t}^{i}, S_{t}^{i}\right)+\delta R_{1}^{o}\left(S_{t}^{i}, S_{t}^{x}\right) \cdot \delta J_{1}^{i}\left(\mathbf{P}_{\mathbf{t}+\mathbf{2}}\right)-\lambda_{t}^{i}
$$

We consider unilateral reductions of the slack of $i$ starting from a steady state so that $R_{1}^{o}\left(S_{t}^{i}, S_{t}^{x}\right)=1$. Furthermore, with $J_{1}^{i}\left(\mathbf{P}_{\mathbf{t}+\mathbf{2}}\right)=1+\lambda_{t+2}^{i}$ (Lemma 4 in the appendix to this chapter) and $F_{2}\left(P_{t}^{i}, S_{t}^{i}\right)=B^{\prime}-1$, it follows:

$$
0=B^{\prime}-1+\delta^{2}\left(1+\lambda_{t+2}^{i}\right)-\lambda_{t}^{i}
$$

As the optimization problem is the same in every period in a steady state equilibrium, $\lambda_{t}^{i}=\lambda_{t+2}^{i}=\lambda$. Solving for $B^{\prime}$ yields the implicit solution for $S^{*}$ :

$$
B^{\prime}=1-\delta^{2}+\left(1-\delta^{2}\right) \lambda
$$

This condition summarizes the tradeoff between marginal benefits and marginal costs of decreasing slack. The less patient firm $i$ is, so the more weight it puts on instantaneous payoff, i.e. the smaller $\delta$ is, the greater is $B^{\prime}$ and with $B^{\prime \prime}<0$ the smaller is the slack $i$ chooses. Therefore, if $\delta$ decreases, the firm cares less about slack in the future but grasps profit today. A more detailed intuition based on an infinite geometric series is given in the appendix to this chapter. If $\lambda>0$,

\footnotetext{
${ }^{41}$ Derivatives would be greater than zero for further decreases of their slack though.
} 
the constraint must be binding from the complementary slackness conditions. For equation (5.28) to hold, the greater $\lambda$ is, the greater must be $B^{\prime}$ and, as $B^{\prime \prime}<0$, the smaller must be the slack. If the constraint is binding, firm $i$ has to choose a smaller slack than it would otherwise do. Conversely, if $\lambda$ is zero, the solution to the constrained maximization problem is equal to the solution to the unconstrained maximization problem, i.e. the slack $S^{M *}$ that firm $i$ chooses in equilibrium if all prices are sufficiently high. Consequently, the implicit definition for $S^{M *}$ is given by

$$
B^{\prime}=1-\delta^{2}
$$

Average yardstick regulation. Under this regulatory regime, all relevant derivatives of the regulatory rule are always $\frac{1}{2}$ as each price is the average of two slacks (see appendix). As furthermore in all steady state equilibria, the FOC must hold in every period, we can update the FOC, equation (5.15), by one period and plug it into (5.25) to find

$$
0=F_{2}\left(P_{t}^{i}, S_{t}^{i}\right)+\delta \frac{1}{2} \cdot\left(\delta J_{1}^{i}\left(\mathbf{P}_{\mathbf{t + 2}}\right)-F_{2}\left(P_{t+1}^{i}, S_{t+1}^{i}\right)+\lambda_{t+1}^{i}\right)-\lambda_{t}^{i}
$$

Applying the same reasoning as above with $S_{t+1}^{i}=S_{t}^{i}=S^{*}, J_{1}^{i}\left(\mathbf{P}_{\mathbf{t}+\mathbf{2}}\right)=1+\lambda_{t+2}^{i}$, $F_{2}\left(P_{t}^{i}, S_{t}^{i}\right)=B^{\prime}-1$ and $\lambda=\lambda_{t}=\lambda_{t+1}^{i}=\lambda_{t+2}^{i}$ we find

$$
0=B^{\prime}\left(1-\frac{1}{2} \delta\right)+1\left(\frac{1}{2} \delta^{2}+\frac{1}{2} \delta-1\right)+\lambda\left(\frac{1}{2} \delta^{2}+\frac{1}{2} \delta-1\right)
$$

and solving for $B^{\prime}$, it follows the implicit solution for $S^{*}$ :

$$
B^{\prime}=\frac{\left(1-\frac{1}{2} \delta^{2}-\frac{1}{2} \delta\right)+\lambda\left(1-\frac{1}{2} \delta^{2}-\frac{1}{2} \delta\right)}{1-\frac{1}{2} \delta} .
$$

Under average yardstick regulation, $B^{\prime}$ also decreases in $\delta$ and hence the slack $i$ chooses increases in the weight the firm puts on future payoff. Again $B^{\prime}$ increases in $\lambda$ so the slack chosen if the constraint is binding is smaller than the slack chosen if all prices are sufficiently high. The solution to the corresponding unconstrained 
maximization problem, i.e. the slack $S^{A *}$ firm $i$ chooses in equilibrium if all prices are sufficiently high, does not include $\lambda$. So, $S^{A *}$ is implicitly defined by

$$
B^{\prime}=1-\frac{\frac{1}{2} \delta^{2}}{1-\frac{1}{2} \delta} \text {. }
$$

Inspection of equations (5.28) and (5.32) reveals that no slack higher than $S^{M *}$ and $S^{A *}$ can exist in a steady state under the respective regulatory regime. As $B^{\prime \prime}<0$ and $\lambda$ is nonnegative from the complementary slackness conditions, neither (5.28) nor (5.32) could hold in any steady state with slack greater than $S^{M *}$ and $S^{A *}$ respectively. In such a steady state, marginal benefits of unilaterally reducing slack would be greater than marginal costs of doing so. Consequently, firm $i$ would unilaterally deviate by reducing its slack, which contradicts the existence of such punishment-free Markov-perfect steady state equilibria. This leads to the following proposition that is directly derived from the analysis above:

\section{Proposition 1.}

(i) In any punishment-free Markov-perfect steady state equilibrium under frontier yardstick regulation the slack is between 0 and $S^{M *}, S^{*} \in\left[0, S^{M *}\right]$.

(ii) In any punishment-free Markov-perfect steady state equilibrium under average yardstick regulation the slack is between 0 and $S^{A *}, S^{*} \in\left[0, S^{A *}\right]$.

\subsubsection{Steady state equilibria}

From Lemma 1, it followed that there cannot exist any asymmetric steady state equilibrium. It is straightforward that the analysis above can analogously be done for firms $o$ and $x$. Taking the optimal strategies of firms $o$ and $x$ as given, we show that it is optimal for $i$ to follow the same strategy. By doing this, we prove the existence of the equilibria characterized above.

Assume optimal Markov-perfect strategies of firms $o$ and $x$ under frontier yardstick regulation are given by

$$
f^{o}\left(\mathbf{P}_{\mathbf{t}}\right)=f^{x}\left(\mathbf{P}_{\mathbf{t}}\right)=S^{*}=\min \left(S^{M *}, P_{t}^{i}, P_{t}^{o}, P_{t}^{x}\right) .
$$


So, firms $o$ and $x$ choose $S^{M *}$ or at least one firm $j=i, o, x$ cannot choose any higher slack without violating the break even constraint given $\mathbf{P}_{\mathbf{t}}$. In the latter case, this firm's choice of slack would remain optimal if the other firms chose higher slacks instead. ${ }^{42}$ Furthermore, in all steady states with slack greater than $S^{M *}$, firms would unilaterally deviate by reducing slack. Consequently, if the above strategies constitute an equilibrium, it is punishment-free.

Given the above strategies, it cannot be optimal for firm $i$ to choose any slack greater than $S^{M *}$ as it could reduce its slack to $S^{M *}$ without affecting any price in $t+1$. As $F_{2}\left(P_{t}^{i}, S_{t}^{i}\right)<0$, this would result in higher instantaneous and intertemporal payoff. The same is true for any $S_{t}^{i}>S_{t}^{o}=S_{t}^{x}$ as $i$ 's slack does not affect future prices if $S_{t}^{i}>S_{t}^{o}=S_{t}^{x}$ from the regulatory rule. Accordingly the FOC cannot hold for $S_{t}^{i}>S_{t}^{o}=S_{t}^{x}$ as $F_{2}\left(P_{t}^{i}, S_{t}^{i}\right)<0$ and $\lambda_{t}^{i}$ is nonnegative from the complementary slackness conditions. Thus, it is never optimal for $i$ to choose a slack higher than $o$ and $x$ under frontier yardstick regulation, and the optimal strategy of $i$ given $\mathbf{P}_{\mathbf{t}}$ and the strategies of $o$ and $x$ must satisfy $f^{i}\left(\mathbf{P}_{\mathbf{t}}\right) \leq \min \left(S^{M *}, P_{t}^{i}, P_{t}^{o}, P_{t}^{x}\right)$.

We now show that this inequality holds with equality: As the value function is concave in the state variables, $F$ is strictly concave in slack and the left hand side derivative of the regulatory rule with respect to the slack of $i$ must be equal to one in all steady states with $S^{*}>0, \lambda>0$ in all steady states with $S^{*}<S^{M *}$. Accordingly the steady state described by $S^{M *}$ is strictly preferred by firm $i$ over all other steady states with lower slack. (Obviously all steady states with positive slack are preferred by $i$ over the one with zero slack.)

From the concavity of the value function and the strict concavity of $F$ concerning slack, it also follows that $\lambda$ decreases in the steady state value of slack for all $S^{*}<S^{M *}$. As a consequence, firm $i$ never unilaterally deviates by reducing slack from a situation where all firms choose the same slack, given $S_{t}^{i} \leq S^{M *}$ : If firm $i$ unilaterally reduces its slack starting from such a situation in $t$, the constraint is not binding in that period, so $\lambda_{t}^{i}$ needs to be zero from the complementary slackness conditions. With the concavity of the value function and strict concavity of $F$ with respect to slack this cannot be optimal as the FOC could not hold. Then

\footnotetext{
${ }^{42}$ In the notation of the definition of punishment-free Markov-perfect equilibria, p. 114, this firm facing the lowest price is labelled firm $l$.
} 
$f^{i}\left(\mathbf{P}_{\mathbf{t}}\right)=S^{*}=\min \left(S^{M *}, P_{t}^{i}, P_{t}^{o}, P_{t}^{x}\right)$ is the optimal strategy given the strategies of $o$ and $x$. As $\min \left(S^{M *}, P_{t}^{i}, P_{t}^{o}, P_{t}^{x}\right)$ can take on every value between zero and $S^{M *}$ depending on initial prices, existence of a punishment-free Markov-perfect steady state equilibrium under frontier yardstick regulation is established for every slack $S^{*} \epsilon\left[0, S^{M *}\right]$.

Assume further that optimal strategies of firms $o$ and $x$ under average yardstick regulation are given by

$$
f^{o}\left(\mathbf{P}_{\mathbf{t}}\right)=f^{x}\left(\mathbf{P}_{\mathbf{t}}\right)=S^{*}=\min \left(S^{A *}, P_{t}^{i}, P_{t}^{o}, P_{t}^{x}\right) .
$$

With the same reasoning as above, it follows that if these strategies constitute an equilibrium, it is punishment-free. The strategies given by equations (5.34) and (5.35), differ only by the unique optimal value of slack, given prices are sufficiently high. Accordingly the corresponding proof for average yardstick regulation is very similar to the one above. It is not optimal for firm $i$ to choose a slack higher than the one $o$ and $x$ choose given their above strategies: First, note that under average yardstick regulation, all relevant derivatives of the regulatory rule are equal to $\frac{1}{2}$ as every price is the average of the slacks of the other two firms of the period before. Now consider $S_{t}^{i}>S_{t}^{o}=S_{t}^{x}$ : neither $o$ nor $x$ would choose a higher slack in $t+1$ than in $t$ as then $\min \left(P_{t+1}^{i}, P_{t+1}^{o}, P_{t+1}^{x}\right)=P_{t+1}^{i}=S_{t}^{o}=S_{t}^{x}$. It follows that the highest possible slack from $t+2$ on would not be greater than $S_{t}^{o}=S_{t}^{x}$ for all slacks $S_{t}^{i}>S_{t}^{o}=S_{t}^{x}$. As $F_{2}\left(P_{t}^{i}, S_{t}^{i}\right)<0, i$ could increase its instantaneous and intertemporal payoff by decreasing its slack and choosing $S_{t}^{i}=S_{t}^{o}=S_{t}^{x}$. The rest of the proof is a straightforward repetition of the arguments above using $S^{A *}$ and the corresponding derivatives of the regulatory rule. 
We summarize these findings in the following proposition:

\section{Proposition 2.}

(i)

Under frontier yardstick regulation, the triple of strategies $\left(f^{i}\left(\mathbf{P}_{\mathbf{t}}\right), f^{o}\left(\mathbf{P}_{\mathbf{t}}\right), f^{x}\left(\mathbf{P}_{\mathbf{t}}\right)\right)$ with $f^{j}\left(\mathbf{P}_{\mathbf{t}}\right)=S^{*}=\min \left(S^{M *}, P_{t}^{i}, P_{t}^{o}, P_{t}^{x}\right), j=i, o, x$, constitutes a punishmentfree Markov-perfect steady state equilibrium. Every slack $S^{*} \epsilon\left[0, S^{M *}\right]$ can exist in equilibrium and $S^{M *}$ offers the highest intertemporal payoff for firms.

(ii)

Under average yardstick regulation, the triple of strategies $\left(f^{i}\left(\mathbf{P}_{\mathbf{t}}\right), f^{o}\left(\mathbf{P}_{\mathbf{t}}\right), f^{x}\left(\mathbf{P}_{\mathbf{t}}\right)\right)$ with $f^{j}\left(\mathbf{P}_{\mathbf{t}}\right)=S^{*}=\min \left(S^{A *}, P_{t}^{i}, P_{t}^{o}, P_{t}^{x}\right), j=i, o, x$, constitutes a punishmentfree Markov-perfect steady state equilibrium. Every slack $S^{*} \epsilon\left[0, S^{A *}\right]$ can exist in equilibrium and $S^{A *}$ offers the highest intertemporal payoff for firms.

It is important to note that the regulator cannot induce the zero slack steady state by simply setting all prices to zero. In our analysis, necessary costs have been normalized to zero. However, the reason why regulatory schemes like yardstick regulation exist essentially is that the regulator does not know how large necessary costs of production are. Otherwise, she could directly mandate optimal prices without applying yardstick regulation. By exogenously setting too low prices in the first regulatory period, the regulator risks firms going bankrupt, as they cannot break even anymore. While it is not explicitly modeled here, it seems reasonable that it is crucial to the regulator that firms subject to regulation, producing without slack, can cover their real and necessary costs. One could think of a large welfare loss outside of the model that is associated with firms, that provide essential services, not being able to cover their real and necessary costs.

Given this restriction and that no slack higher than $S^{M *}$ and $S^{A *}$ under the respective regime can exist in a steady state, it seems reasonable that the regulator initially sets prices which are relatively high. Therefore, comparing the upper ends of the intervals of feasible steady state slacks seems particularly relevant. 


\subsubsection{Comparative dynamics}

From Propositions 1 and 2, we know that every slack between 0 and $S^{M *}$ under frontier yardstick regulation and between 0 and $S^{A *}$ under average yardstick regulation can describe a steady state equilibrium. Furthermore, we know that there cannot exist punishment-free Markov-perfect steady state equilibria with higher slack under the respective regulatory regime. By comparing the implicit solutions for $S^{M *}$ and $S^{A *}$, we find that all punishment-free Markov-perfect steady state equilibria under average yardstick regulation can be equilibria under frontier yardstick regulation while the reverse is not true. This leads to the following proposition:

Proposition 3. The highest slack that can be realized in a punishment-free Markovperfect steady state equilibrium is greater under frontier yardstick regulation than under average yardstick regulation.

Proof. $S^{A *}$ is implicitly defined by (5.33) and the corresponding value under frontier yardstick regulation, $S^{M *}$, is implicitly defined by (5.29). As $B^{\prime}>0$ and $B^{\prime \prime}<0$, $S^{M *}>S^{A *}$ if the following inequality holds:

$$
1-\frac{\frac{1}{2} \delta^{2}}{1-\frac{1}{2} \delta}>1-\delta^{2} .
$$

Rearranging yields

$$
1>\delta
$$

Hence, inequality (5.36) always holds.

Intuitively, orientation at the performance of 'the best' of all other firms rather than the average of all other firms to define constraints for a firm under yardstick regulation seems to be the tougher regulation. Incentives to produce efficiently, i.e. without slack, should be strong. Proposition 3 questions this intuition. Using historical cost data of other firms allows each firm to influence the own yardstick. As this influence is greater under frontier yardstick regulation all firms could be less willing to 'push' the other firms because they will have to 'push back' in return. 


\subsection{Conclusion}

While Shleifer's (1985) version of yardstick regulation uses current performance of other firms to find current constraints for an evaluated firm, real life applications of yardstick regulation frequently define constraints, e.g. prices allowed to be charged, ex ante based on data from the regulatory period(s) before. This use of historical cost data in yardstick regulation enables a firm to affect the price it can charge in the future. Affecting other firms' constraints and thus behavior, the current performance of a firm is directly linked to its own future constraints.

This analysis showed in a simple model framework that inefficient steady state equilibria in which all firms choose positive slack can exist under yardstick regulation without any form of collusion if historical cost data is used. Furthermore, the highest slack that can exist in a punishment-free Markov-perfect steady state equilibrium is higher under frontier yardstick regulation, where the firm with the lowest costs of all but the evaluated firm defines the yardstick, than if the average of all other firms is used. This challenges the perception that incentives to produce efficiently are strongest if the best of all other firms is the yardstick in a yardstick regulation using historical cost data. 


\section{Appendix to Chapter 5}

\section{Proof of Lemma 1}

As regulatory rules are anonymous and $C$ is normalized to zero, only 5 relevant different cases can be distinguished, potentially with indices changed and updated though:

(I) $P_{t}^{i}=P_{t}^{o}=P_{t}^{x}=S_{t}^{i}=S_{t}^{o}=S_{t}^{x}$

(II) $S_{t}^{i}=S_{t}^{o}>S_{t}^{x}$

(III) $S_{t}^{i}>S_{t}^{o}=S_{t}^{x}$

(IV) $S_{t}^{i}>S_{t}^{o}>S_{t}^{x}$

(V) $S_{t}^{i}=S_{t}^{o}=S_{t}^{x}<P_{t}^{i}=P_{t}^{o}=P_{t}^{x}$

The reasoning is explained below in detail for case (II) under frontier yardstick regulation and average yardstick regulation, the remaining is then a straightforward application along these lines.

Case (I):

If all three prices and all three slacks are the same in $t$ the regulatory rule does not force any change. Prices in $t+1$ are the same as in $t$ and the same slack as in $t$ is possible for all firms.

Frontier yardstick regulation

Case (II):

$$
P_{t+1}^{i}=P_{t+1}^{o}=S_{t}^{x}<P_{t+1}^{x}=S_{t}^{i}=S_{t}^{o}
$$




$$
\begin{aligned}
& \Rightarrow \\
S_{t+1}^{i} & \leq S_{t}^{x} \\
S_{t+1}^{o} & \leq S_{t}^{x} \\
S_{t+1}^{x} & \leq S_{t}^{i}=S_{t}^{o} \\
& \Rightarrow \\
S_{t+2}^{i} & \leq P_{t+2}^{i} \leq S_{t}^{x} \\
S_{t+2}^{o} & \leq P_{t+2}^{o} \leq S_{t}^{x} \\
S_{t+2}^{x} & \leq P_{t+2}^{x} \leq S_{t}^{x}
\end{aligned}
$$

So in $t+2$, no slack higher than the smallest of $t$ can be chosen. Then either case (I) or one of the cases (II)-(V) applies.

Under frontier yardstick regulation, the price that a firm is allowed to charge is the minimum of the slacks the other two firms chose in the period before. Therefore, if firms $i$ and $o$ choose the same slack in $t$ and firm $x$ chooses a smaller one, the price $i$ and $o$ are allowed to charge in $t+1$ is equal to $S_{t}^{x}$ while $P_{t+1}^{x}$ is equal to the slack $i$ and $o$ choose in $t$. In $t+1, x$ may, consequently, choose any slack that is not greater than $P_{t+1}^{x}=S_{t}^{i}=S_{t}^{o}$. The slack $i$ and $o$ can choose is not greater than $P_{t+1}^{i}=P_{t+1}^{o}=S_{t}^{x}$ and hence smaller than $P_{t+1}^{x}=S_{t}^{i}=S_{t}^{o}$. In $t+2$, the price $i, o$ and $x$ may charge is not greater than the smallest slack in $t$, i.e. $S_{t}^{x}$. Only one firm, $x$, can choose a higher slack than this in $t+1$. But even if it does so, the smaller one of any two slacks chosen in $t+1$ cannot be greater than $S_{t}^{x}$. In $t+2$, either all three firms choose the same slack and this slack is equal to the price they may charge or one of the cases (II) to (V) applies. 
Case (III):

$$
\begin{aligned}
& S_{t+1}^{i} \leq P_{t+1}^{i}=S_{t}^{x}=S_{t}^{o} \\
& S_{t+1}^{o} \leq P_{t+1}^{o}=S_{t}^{x}=S_{t}^{o} \\
& S_{t+1}^{x} \leq P_{t+1}^{x}=S_{t}^{o}=S_{t}^{x}
\end{aligned}
$$

So in $t+1$, no slack higher than the smallest of $t$ can be chosen. Then either case (I) or one of the cases (II)-(V) applies.

Case (IV):

$$
\begin{aligned}
P_{t+1}^{i} & =S_{t}^{x} \\
P_{t+1}^{o} & =S_{t}^{x} \\
P_{t+1}^{x} & =S_{t}^{o} \\
& \Rightarrow \\
& \\
S_{t+1}^{i} & \leq S_{t}^{x} \\
S_{t+1}^{o} & \leq S_{t}^{x} \\
S_{t+1}^{x} & \leq S_{t}^{o} \\
& \Rightarrow \\
S_{t+2}^{i} & \leq P_{t+2}^{i} \leq S_{t}^{x} \\
S_{t+2}^{o} & \leq P_{t+2}^{o} \leq S_{t}^{x} \\
S_{t+2}^{x} & \leq P_{t+2}^{x} \leq S_{t}^{x}
\end{aligned}
$$

So in $t+2$, no slack higher than the smallest of $t$ can be chosen. Then either case (I) or one of the cases (II)-(V) applies. 
Case (V):

$$
P_{t+1}^{i}=P_{t+1}^{o}=P_{t+1}^{x}=S_{t}^{i}=S_{t}^{o}=S_{t}^{x}
$$

then either case (I) or one of the cases (II)-(V) applies.

Average yardstick regulation

Case (II):

$$
\begin{aligned}
P_{t+1}^{i} & =\frac{S_{t}^{o}+S_{t}^{x}}{2} \\
P_{t+1}^{o} & =\frac{S_{t}^{i}+S_{t}^{x}}{2} \\
P_{t+1}^{x} & =\frac{S_{t}^{i}+S_{t}^{o}}{2}=S_{t}^{i}=S_{t}^{o} \\
& \Rightarrow \quad \\
S_{t+1}^{i} & \leq \frac{S_{t}^{o}+S_{t}^{x}}{2}<S_{t}^{i} \\
S_{t+1}^{o} & \leq \frac{S_{t}^{i}+S_{t}^{x}}{2}<S_{t}^{o} \\
S_{t+1}^{x} & \leq \frac{S_{t}^{i}+S_{t}^{o}}{2}=S_{t}^{i}=S_{t}^{o}\left[\mathrm{and} S_{t+1}^{x} \gtreqless S_{t}^{x}\right] \\
& \Rightarrow \quad \leq P_{t+2}^{i} \leq \frac{S_{t}^{i}+S_{t}^{x}+S_{t}^{i}+S_{t}^{o}}{4}<S_{t}^{i}=S_{t}^{o} \\
S_{t+2}^{i} & \leq P_{t}^{o}+S_{t}^{o} \\
S_{t+2}^{o} & \leq P_{t+2}^{o} \leq \frac{S_{t}^{o}+S_{t}^{x}=S_{t}^{i}}{4} \\
S_{t+2}^{x} & \leq P_{t+2}^{x} \leq \frac{S_{t}^{o}+S_{t}^{x}+S_{t}^{i}+S_{t}^{x}}{4}<S_{t}^{i}=S_{t}^{o}
\end{aligned}
$$

So the highest slack chosen in $t$ cannot be chosen by anyone in $t+2$. Then either case (I) or one of the cases (II)-(V) applies. 
Under average yardstick regulation, the price that a firm may charge is equal to the average of the slacks that the other two firms chose in the period before. So, if firms $i$ and $o$ choose the same slack in $t$ and firm $x$ chooses a smaller slack, the price $i$ and $o$ are allowed to charge in $t+1$ is smaller than the one $x$ may charge and smaller than the slack $i$ and $o$ choose in $t$. Accordingly both have to choose a smaller slack in $t+1$. In $t+1, x$ may choose a slack that is greater than $S_{t}^{x}$ but not greater than the slack $i$ and $o$ choose in $t$. In $t+2$, all prices are smaller than the greatest slack in $t$ so that this slack cannot be chosen anymore. Then either all three firms choose the same slack and this slack is equal to the price they may charge or one of the cases (II) to (V) applies.

Case (III):

$$
\begin{aligned}
P_{t+1}^{i} & =\frac{S_{t}^{o}+S_{t}^{x}}{2}=S_{t}^{o}=S_{t}^{x} \\
P_{t+1}^{o} & =\frac{S_{t}^{i}+S_{t}^{x}}{2} \\
P_{t+1}^{x} & =\frac{S_{t}^{i}+S_{t}^{o}}{2} \\
& \Rightarrow \\
S_{t+1}^{i} & \leq \frac{S_{t}^{o}+S_{t}^{x}}{2}=S_{t}^{o}=S_{t}^{x}<S_{t}^{i} \\
S_{t+1}^{o} & \leq \frac{S_{t}^{i}+S_{t}^{x}}{2}<S_{t}^{i}\left[\text { and } S_{t+1}^{o} \gtreqless S_{t}^{o}\right] \\
S_{t+1}^{x} & \leq \frac{S_{t}^{i}+S_{t}^{o}}{2}<S_{t}^{i}\left[\text { and } S_{t+1}^{x} \gtreqless S_{t}^{x}\right]
\end{aligned}
$$

So the highest slack chosen in $t$ cannot be chosen by anyone in $t+1$. Then either case (I) or one of the cases (II)-(V) applies. 
Case (IV):

$$
\begin{aligned}
P_{t+1}^{i} & =\frac{S_{t}^{o}+S_{t}^{x}}{2} \\
P_{t+1}^{o} & =\frac{S_{t}^{i}+S_{t}^{x}}{2} \\
P_{t+1}^{x} & =\frac{S_{t}^{i}+S_{t}^{o}}{2} \\
& \Rightarrow \\
S_{t+1}^{i} & \leq \frac{S_{t}^{o}+S_{t}^{x}}{2}<S_{t}^{i} \\
S_{t+1}^{o} & \leq \frac{S_{t}^{i}+S_{t}^{x}}{2}<S_{t}^{i} \\
S_{t+1}^{x} & \leq \frac{S_{t}^{i}+S_{t}^{o}}{2}<S_{t}^{i}
\end{aligned}
$$

So the highest slack chosen in $t$ cannot be chosen by anyone in $t+1$. Then either case (I) or one of the cases (II)-(V) applies.

Case (V):

$$
\begin{aligned}
P_{t+1}^{i} & =\frac{S_{t}^{o}+S_{t}^{x}}{2}=P_{t+1}^{o}=\frac{S_{t}^{i}+S_{t}^{x}}{2}=P_{t+1}^{x}=\frac{S_{t}^{i}+S_{t}^{o}}{2}=S_{t}^{i}=S_{t}^{o}=S_{t}^{x} \\
& \Rightarrow \\
S_{t+1}^{i} & \leq P_{t+1}^{i}=S_{t}^{i}=S_{t}^{o}=S_{t}^{x} \\
S_{t+1}^{o} & \leq P_{t+1}^{o}=S_{t}^{o}=S_{t}^{i}=S_{t}^{x} \\
S_{t+1}^{x} & \leq P_{t+1}^{x}=S_{t}^{x}=S_{t}^{i}=S_{t}^{o}
\end{aligned}
$$

Then either case (I) or one of the cases (II)-(V) applies.

As long as slacks differ in period $t$, in $t+2$ at the latest, the highest slack of $t$ cannot be chosen by any firm anymore under both regulatory regimes. (Under 
frontier yardstick regulation, at the latest in $t+2$, no slack higher than the smallest of $t$ can be chosen.) Consequently, the maximum of the three slacks monotonically decreases, potentially with a delay that is not greater than two periods. Furthermore, all slacks are bounded below at zero. It follows that slacks necessarily have to converge. As the price for each firm is in every period the minimum or the average of the slacks of the other two firms in the period before, prices converge too. Prices and slacks cannot converge to different values so that profits of all firms must be zero in every steady state.

\section{Derivatives of the regulatory rules}

Frontier yardstick regulation

We focus on the example of the price firm $i$ can charge in $t+1$. The corresponding derivatives regarding reductions of slack for the other firms and for all other periods are found analogously. The regulatory rule is given by (5.4):

$$
P_{t+1}^{i}=C+\min \left(S_{t}^{o}, S_{t}^{x}\right)=R^{i}\left(S_{t}^{o}, S_{t}^{x}\right)
$$

The relevant left hand side derivatives for $S_{t}^{o} \neq S_{t}^{x}$ are given by

$$
R_{1}^{i}\left(S_{t}^{o}, S_{t}^{x}\right)=\frac{\partial R^{i}\left(S_{t}^{o}, S_{t}^{x}\right)}{\partial S_{t}^{o}}= \begin{cases}1 & \text { for } S_{t}^{o}<S_{t}^{x} \\ 0 & \text { for } S_{t}^{o}>S_{t}^{x}\end{cases}
$$

and

$$
R_{2}^{i}\left(S_{t}^{o}, S_{t}^{x}\right)=\frac{\partial R^{i}\left(S_{t}^{o}, S_{t}^{x}\right)}{\partial S_{t}^{x}}= \begin{cases}0 & \text { for } S_{t}^{o}<S_{t}^{x} \\ 1 & \text { for } S_{t}^{o}>S_{t}^{x}\end{cases}
$$

Starting from $S_{t}^{o}=S_{t}^{x}$ and for a constant slack of the respective other firm, the left hand side derivative is equal to one for both firms. However, the price firm $i$ is allowed to charge in $t+1$ is reduced by one marginal unit if either firm $o$ or firm $x$ or both firms simultaneously reduce their respective slack in $t$ by one marginal unit. 
In particular, slightly abusing notation, we have

$$
R_{1}^{i}\left(S_{t}^{o}, S_{t}^{x}\right)+R_{2}^{i}\left(S_{t}^{o}, S_{t}^{x}\right)=\frac{\partial R^{i}\left(S_{t}^{o}, S_{t}^{x}\right)}{\partial S_{t}^{o}}+\frac{\partial R^{i}\left(S_{t}^{o}, S_{t}^{x}\right)}{\partial S_{t}^{x}}=1 \text { for all } S_{t}^{o}, S_{t}^{x}
$$

For simultaneous reductions of the slacks of both firms, we are clearly not holding the respective other slack constant. However, as for simultaneous changes in the slacks it is unimportant for our result whether the change of the slack of firm $o$ or of $x$ or of both change the constraint of firm $i$, we refrain from introducing additional notation that does not provide further insights.

To derive (5.39) for $S_{t}^{o}=S_{t}^{x}$ and simultaneous changes of slack of $o$ and $x$, let

$$
\widetilde{S_{t}^{o}}=S_{t}^{o}+\epsilon
$$

and

$$
\widetilde{S_{t}^{x}}=S_{t}^{x}+\epsilon,
$$

where $\epsilon \neq 0$.

For $S_{t}^{o}=S_{t}^{x}$ and $\widetilde{S_{t}^{o}}=\widetilde{S_{t}^{x}}$ we see that

$$
\min \left(S_{t}^{o}, S_{t}^{x}\right)=S_{t}^{o}=S_{t}^{x} \text { and } \min \left(\widetilde{S_{t}^{o}}, \widetilde{S_{t}^{x}}\right)=\widetilde{S_{t}^{o}}=\widetilde{S_{t}^{x}}
$$

then

$$
\min \left(\widetilde{S_{t}^{o}}, \widetilde{S_{t}^{x}}\right)-\min \left(S_{t}^{o}, S_{t}^{x}\right)=\epsilon
$$

In analogy to the definition of the derivative, we find

$$
\lim _{\epsilon \rightarrow 0} \frac{\min \left(S_{t}^{o}+\epsilon, S_{t}^{x}+\epsilon\right)-\min \left(S_{t}^{o}, S_{t}^{x}\right)}{\epsilon}=1 .
$$




\section{Average yardstick regulation}

The regulatory rule is given by (5.5):

$$
P_{t+1}^{i}=\frac{1}{2} \sum_{j \neq i}\left(C+S_{t}^{j}\right)=C+\frac{1}{2} \sum_{j \neq i} S_{t}^{j}=R^{i}\left(S_{t}^{o}, S_{t}^{x}\right) .
$$

Consequently, all changes in slack of any firm will result in changes in the prices the other two firms may charge in the following period by half of the magnitude of the aforementioned change. Spelled out for the price firm $i$ can charge in $t+1$ this is

$$
R_{1}^{i}\left(S_{t}^{o}, S_{t}^{x}\right)=R_{2}^{i}\left(S_{t}^{o}, S_{t}^{x}\right)=\frac{1}{2}
$$

Under average yardstick regulation, all other derivatives of the regulatory rule with respect to one of the two relevant slacks are equal to $\frac{1}{2}$, too.

\section{Proof of Lemma 2}

Recall the FOC, equation (5.15),

$$
F_{2}\left(P_{t}^{i}, S_{t}^{i}\right)+\delta J_{2}^{i}\left(\mathbf{P}_{\mathbf{t}+\mathbf{1}}\right) \cdot R_{1}^{o}\left(S_{t}^{i}, S_{t}^{x}\right)+\delta J_{3}^{i}\left(\mathbf{P}_{\mathbf{t}+\mathbf{1}}\right) \cdot R_{1}^{x}\left(S_{t}^{i}, S_{t}^{o}\right)-\lambda_{t}^{i}=0
$$

and complementary slackness conditions (5.14):

$$
\lambda_{t}^{i} \geq 0 \text { and } \lambda_{t}^{i}\left(P_{t}^{i}-S_{t}^{i}\right)=0 .
$$

Assume firms are in a steady state so that $P_{t}^{i}=P_{t}^{o}=P_{t}^{x}=S_{t}^{i}=S_{t}^{o}=S_{t}^{x}$ and $P_{t}^{i} \leq S^{M *}$ under frontier yardstick regulation and $P_{t}^{i} \leq S^{A *}$ under average yardstick regulation. If $i$ 's choice of slack is optimal, the FOC and complementary slackness conditions must hold.

Now, assume one of the other firms, e.g. firm $o$, instead chooses a marginally smaller slack in $t$ so that $P_{t+1}^{i}=P_{t+1}^{x}<P_{t+1}^{o}$ and $P_{t+1}^{i}<S^{M *}$ under frontier yardstick regulation and $P_{t+1}^{i}<S^{A *}$ under average yardstick regulation. From the break even condition, we know that $i$ has to reduce its slack by at least the marginal change of the price that it may charge in $t+1$ so that the left hand side derivative 
of $f^{i}\left(\mathbf{P}_{\mathbf{t}+\mathbf{1}}\right)$ with respect to $i$ 's own price cannot be smaller than one. Clearly, the sum of the left hand side derivatives $f_{1}^{i}\left(\mathbf{P}_{\mathbf{t}+\mathbf{1}}\right)+f_{3}^{i}\left(\mathbf{P}_{\mathbf{t}+\mathbf{1}}\right) \geq 1$ too. (Throughout this chapter, we are only considering reductions of slack.)

As we require equilibria to be punishment-free, this equation holds with equality and $S_{t+1}^{i}=P_{t+1}^{i}$. To show this, we fix the slacks $o$ and $x$ at their respective highest admissible value of slack in $t+1, S_{t+1}^{o}=P_{t+1}^{o}$ and $S_{t+1}^{x}=P_{t+1}^{x}$. Consequently, the respective left hand side derivatives of the regulatory rule for firm $i$ are the same as in a steady state as $i$ cannot choose a slack higher than its price and thus $S_{t+1}^{i}=\min \left(S_{t+1}^{i}, S_{t+1}^{o}, S_{t+1}^{x}\right)$. If firm $i$ decreases its slack by even more than the marginal change of its price to any $\underline{S}_{t+1}^{i}<P_{t+1}^{i}$, the constraint is not binding in $t+1$. It follows that $\lambda_{t+1}^{i}=0$ from the complementary slackness conditions. With $\underline{S}_{t+1}^{i}<S_{t}^{i}$, it also follows that $F_{2}\left(P_{t+1}^{i}, \underline{S}_{t+1}^{i}\right)>F_{2}\left(P_{t}^{i}, S_{t}^{i}\right)$ as $F$ is strictly concave in slack. Besides, the value function is concave in the state variables so that $J_{2}^{i}\left(\mathbf{P}_{\mathbf{t}+\mathbf{2}}\right)$ and $J_{3}^{i}\left(\mathbf{P}_{\mathbf{t}+\mathbf{2}}\right)$ are not smaller than the corresponding derivatives in the initial steady state, where the FOC held, as prices are not greater than in that steady state. Hence, the FOC cannot hold in $t+1$ so that $\underline{S}_{t+1}^{i}$ is not the optimal choice of $i$. Thus, $i$ does not unilaterally reduce its slack by more than what is forced by the reduction of its price in this setting, i.e. $S_{t+1}^{i}=P_{t+1}^{i}$.

We can apply the same reasoning as above for firms $o$ and $x$ to show that no firm unilaterally chooses a slack in $t+1$ that is smaller than $P_{t+1}^{i}=P_{t+1}^{x}$ if the other two firms choose their respective highest admissible slack here. As we require equilibria to be punishment-free, firms do not coordinatedly choose a uniform smaller slack because of other firms choosing this uniform slack. It follows that $f_{1}^{i}\left(\mathbf{P}_{\mathbf{t}+\mathbf{1}}\right)+f_{3}^{i}\left(\mathbf{P}_{\mathbf{t}+\mathbf{1}}\right)=1$ in this setting.

Symmetrically the same reasoning applies for all firms with indices changed.

From the proof above, we can clearly point out the vulnerability of yardstick regulation against the threat of joint mutual punishment and collusion in general. As shown above, it is not optimal in this case for firm $i$ to unilaterally choose any slack smaller than the price that it can charge in $t+1$. However, for example, if at least one other firm chose a smaller slack than this slack under frontier yardstick regulation, it would be optimal for $i$ to do so too. As this applies for all firms, allowing for 
coordination like joint mutual punishment could lead to $f_{1}^{i}\left(\mathbf{P}_{\mathbf{t}+\mathbf{1}}\right)+f_{3}^{i}\left(\mathbf{P}_{\mathbf{t}+\mathbf{1}}\right)>1$. This would give room to equilibria with much higher slack than $S^{M *}$ under frontier yardstick regulation and $S^{A *}$ under average yardstick regulation by increasing costs of reducing slack for all firms.

\section{Lemma 3.}

(i) Frontier yardstick regulation:

If $P_{t}^{i}=P_{t}^{x}<P_{t}^{o}$ and $P_{t}^{i} \leq S^{M *}$, then $f_{2}^{i}\left(\mathbf{P}_{\mathbf{t}}\right)=0$.

(ii) Average yardstick regulation:

If $P_{t}^{i}=P_{t}^{x}<P_{t}^{o}$ and $P_{t}^{i} \leq S^{A *}$, then $f_{2}^{i}\left(\mathbf{P}_{\mathbf{t}}\right)=0$.

Proof. Assume firms are in a steady state so that $P_{t}^{i}=P_{t}^{o}=P_{t}^{x}=S_{t}^{i}=S_{t}^{o}=S_{t}^{x}$ and $P_{t}^{i} \leq S^{M *}$ under frontier yardstick regulation and $P_{t}^{i} \leq S^{A *}$ under average yardstick regulation. As $i$ 's choice of slack is optimal, the FOC, equation (5.15), and complementary slackness conditions (5.14) must hold.

Now, assume that instead the price relevant for one of the firms, e.g. firm $o$, is higher $\bar{P}_{t}^{o}>P_{t}^{i}=P_{t}^{x}$. Applying the same reasoning as in the proof of Lemma 2, it follows that no firm unilaterally chooses a smaller slack than $P_{t}^{i}=P_{t}^{x}$ in this setting. Furthermore, in a punishment-free equilibrium, firms do not coordinatedly choose a uniform (lower) slack that no firm would choose unilaterally if all other firms were to choose higher slacks. Firm $i$ cannot choose any slack higher than its price because of the break even constraint: this implies that $S_{t}^{i}=P_{t}^{i}$.

Hence, the optimal slack of firm $i$ is the same for $\bar{P}_{t}^{o}>P_{t}^{i}=P_{t}^{x}$ and $P_{t}^{o}=P_{t}^{i}=$ $P_{t}^{x}$, with $P_{t}^{i} \leq S^{M *}$ under frontier yardstick regulation and $P_{t}^{i} \leq S^{A *}$ under average yardstick regulation. It directly follows that $f_{2}^{i}\left(\mathbf{P}_{\mathbf{t}}\right)=0$ in this setting.

An intuition for Lemma 3 under frontier yardstick regulation is the following: Firm $i$ knows that the lowest slack in $t$ describes an upper bound for all slacks and prices from $t+2$ onwards. So, as long as the slacks of the other two firms are not smaller than the one $i$ chooses, this upper bound is the same for every slack $o$ and $x$ choose and all prices $o$ and $x$ face. Thus, the marginal benefits and costs of a reduction of slack do not depend on these prices in this setting. Consequently, the decision of $i$ is not affected. Again, the same reasoning applies for $P_{t}^{i}=P_{t}^{o}<P_{t}^{x}$ as well as for firms $O$ and $x$ with changed indices. 


\section{Lemma 4.}

(i) Frontier yardstick regulation:

If $P_{t+2}^{o}=P_{t+2}^{x}<P_{t+2}^{i}$ and $P_{t+2}^{o}=P_{t+2}^{x} \leq S^{M *}$, then $J_{1}^{i}\left(\mathbf{P}^{\mathbf{t}+\mathbf{2}}\right)=1+\lambda_{t+2}^{i}$.

(ii) Average yardstick regulation:

If $P_{t+2}^{o}=P_{t+2}^{x}<P_{t+2}^{i}$ and $P_{t+2}^{o}=P_{t+2}^{x} \leq S^{A *}$, then $J_{1}^{i}\left(\mathbf{P}^{\mathbf{t}+\mathbf{2}}\right)=1+\lambda_{t+2}^{i}$.

Proof. Recall equation (5.18):

$$
\begin{aligned}
J_{1}^{i}\left(\mathbf{P}_{\mathbf{t}}\right)= & F_{1}\left(P_{t}^{i}, S_{t}^{i}\right) \\
& +\delta J_{1}^{i}\left(\mathbf{P}_{\mathbf{t}+\mathbf{1}}\right) \cdot R_{1}^{i}\left(S_{t}^{o}, S_{t}^{x}\right) \cdot f_{1}^{o}\left(\mathbf{P}_{\mathbf{t}}\right)+\delta J_{1}^{i}\left(\mathbf{P}_{\mathbf{t}+\mathbf{1}}\right) \cdot R_{2}^{i}\left(S_{t}^{o}, S_{t}^{x}\right) \cdot f_{1}^{x}\left(\mathbf{P}_{\mathbf{t}}\right) \\
& +\delta J_{2}^{i}\left(\mathbf{P}_{\mathbf{t}+\mathbf{1}}\right) \cdot R_{2}^{o}\left(S_{t}^{i}, S_{t}^{x}\right) \cdot f_{1}^{x}\left(\mathbf{P}_{\mathbf{t}}\right)+\delta J_{3}^{i}\left(\mathbf{P}_{\mathbf{t}+\mathbf{1}}\right) \cdot R_{2}^{x}\left(S_{t}^{i}, S_{t}^{o}\right) \cdot f_{1}^{o}\left(\mathbf{P}_{\mathbf{t}}\right) \\
& +\lambda_{t}^{i} .
\end{aligned}
$$

The way the value function of $i$ is affected by a change of the price that firm $i$ may charge depends on how the other two firms react to this change. Using Lemma 3 for the reactions of $o$ and $x$, inserting $F_{1}\left(P_{t}^{i}, S_{t}^{i}\right)=1$ and updating (5.18) by two periods complete the proof.

Intuitively, Lemma 4 says that if firms are in the steady state equilibrium described by $S^{M *}$, under frontier yardstick regulation, or $S^{A *}$, under average yardstick regulation, and $\lambda=0$, they would not change their slack if their price was higher, but would realize a positive profit that period. Consequently, the discounted sum of the utility of $i$ increases by 1 if the price that firm $i$ is allowed to charge in $t$ increases by one unit. In any steady state equilibrium with a slack smaller than $S^{M *}$ or $S^{A *}$, respectively, we have $\lambda>0$. Hence, firms would like to move to a steady state equilibrium with higher slack, but cannot do so because of the (binding) break even constraint. Reductions of the prices firms can charge then have a larger impact on the intertemporal payoff.

\section{Intuition for $S^{M *}$ based on geometric series}

When firm $i$ decides on the slack in $t$, it considers that its slack defines an upper bound for all prices from $t+2$ onwards under frontier yardstick regulation given 
$S_{t}^{i} \leq \min \left(S_{t}^{o}, S_{t}^{x}\right)$. From the proof of Lemma 2, we know that $o$ and $x$ choose the highest slack that they are allowed to, given $P_{t+1}^{o}=P_{t+1}^{x} \leq P_{t+1}^{i}$ and $P_{t+1}^{o}=P_{t+1}^{x} \leq S^{M *}$, in their optimal decision. Firm $i$ has to trade off profit in $t$ and $t+1$ against slack in $t, t+1, t+2, \ldots, \infty$ when it decides about $S_{t}^{i}=S^{M *}$. (As the price $i$ may charge in $t+1$ is unaffected by $S_{t}^{i}$, it can 'cash in' the profit from reducing slack twice.) In the steady state equilibrium described by $S^{M *}$, implicitly defined by (5.29), marginal costs of reducing slack and marginal benefits of doing so must be equal to each other, so that

$$
1+\delta \cdot 1=\sum_{z=o}^{\infty} \delta^{z} \cdot B^{\prime} .
$$

With $\delta<1$, it follows that

$$
1+\delta \cdot 1=B^{\prime} \frac{1}{1-\delta}
$$

Rearranging yields

$$
B^{\prime}=1-\delta^{2}
$$

which replicates the implicit definition of $S^{M *}$ given by equation (5.29). 


\section{References}

AakviK, A. (2001): Bounding a Matching Estimator: The Case of a Norwegian Training Program. Oxford Bulletin of Economics and Statistics, 63(1), 115-143.

Acemoglu, D. (2009): Introduction to Modern Economic Growth. Princeton: Princeton University Press.

Achen, A. C. and P. N. Courant (2009): What are Grades Made of? Journal of Economic Perspectives, 23(3), 77-92.

Alesina, A. and G.-M. Angeletos (2005): Fairness and Redistribution. American Economic Review, 95(4), 960-980.

Altonji, J. G., T. E. Elder and C. R. Taber (2005): Selection on Observed and Unobserved Variables: Assessing the Effectiveness of Catholic Schools. Journal of Political Economy, 113(1), 151-184.

Andersen, J. J., J. H. Fiva and G. J. Natvik (2014): Voting when the Stakes are High. Journal of Public Economics, 110, 157-166.

Anger, C., V. Demary, O. Koppel and A. Plünnecke (2013): Zu wenig Nachwuchs. iw-dienst, 19, May 9, 2013. http: //www.iwkoeln.de/de/infodienste/ iwd/archiv/beitrag/mint-fachkraefte-zu-wenig-nachwuchs-111260 (accessed September 17, 2014).

Ansell, B. (2014): The Political Economy of Ownership: Housing Markets and the Welfare State. American Political Science Review, 108(2), 383-402. 
Arias Ortiz, E. and C. Dehon (2008): What are the Factors of Success at University? A Case Study in Belgium. CESifo Economic Studies, 54(2), 121-148.

Arulampalam, W., R. A. Naylor and J. P. Smith (2005): Effects of In-Class Variation and Student Rank on the Probability of Withdrawal: Cross-Section and Time-Series Analysis for UK University Students. Economics of Education Review, 24(3), 251-262.

Averch, H. and L. L. Johnson (1962): Behavior of the Firm under Regulatory Constraint. American Economic Review 52(5), 1052-1069.

Balafoutas, L., M. G. Kocher, L. Putterman and M. Sutter (2013): Equality, Equity and Incentives: An Experiment. European Economic Review, 60, $32-51$.

BARon-Boldt, J. (1989): Die Validität von Schulabschlußnoten für die Prognose von Ausbildungs- und Studienerfolg: Eine Metaanalyse nach dem Prinzip der Validitätsgeneralisierung. Europäische Hochschulschriften. Frankfurt am Main: Lang.

Barro, R. J. (1973): The Control of Politicians: An Economic Model. Public Choice, 14(1), 19-42.

Becker, S. O. and M. Caliendo (2006): MHBOUndS: Stata Module to Perform Mantel-Haenszel Bounds to Check Sensitivity of Estimated Average Treatment Effects on the Treated. Version 1.1.5.

Becker, S. O. and M. Caliendo (2007): Sensitivity Analysis for Average Treatment Effects. Stata Journal, 7(1), 71-83.

Becker, S. O. and A. ICHino (2002): Estimation of Average Treatment Effects Based on Propensity Scores. Stata Journal, 2(4), 358-377.

Ben-BAssat, A. and M. DAHAN (2012): Social Identity and Voting Behavior. Public Choice, 151(1-2), 193-214. 
Besley, T. and A. CAse (1995): Incumbent Behavior: Vote-Seeking, Tax-Setting, and Yardstick Competition. American Economic Review, 85(1), 25-45.

Betts, J. R. and D. Morell (1999): The Determinants of Undergraduate Grade Point Average: The Relative Importance of Family Background, High School Resources, and Peer Group Effects. Journal of Human Resources, 34(2), 268-293.

Blackmon, B. G. (1994): Incentive Regulation and the Regulation of Incentives. Boston: Kluwer.

Brennan, G. and J. Buchanan (1984): Voter Choice: Evaluating Political Alternatives. American Behavioral Scientist, 28(2), 185-201.

Brennan, G. and A. Hamlin (1998): Expressive Voting and Electoral Equilibrium. Public Choice, 95(1-2), 149-175.

Burton, L. J. and D. G. Dowling (2005): In Search of the Key Factors that Influence Student Success at University. Proceedings of the 28th HERDSA Annual Conference: Higher Education in a Changing World, 68-78.

Caliendo, M., R. Hujer and S. Thomsen (2008): The Employment Effects of Job-Creation Schemes in Germany: A Microeconometric Evaluation. In T. Fomby, R. C. Hill, D. L. Millimet, J. A. Smith and E. J. Vytlacil (eds.), Advances in Econometrics, 21, 381-428.

Caliendo, M. and S. Kopeinig (2008): Some Practical Guidance for the Implementation of Propensity Score Matching. Journal of Economic Surveys, 22(1), 31-72.

California State Board of Equalization (2012): California Property Tax: An Overview. Publication 29, November 2012. http://www.boe.ca.gov/ proptaxes/pdf/pub29.pdf (accessed September 28, 2014).

Carlsson, F. and O. Johansson-Stenman (2010): Why Do You Vote and Vote as You Do? Kyklos, 63(4), 495-516. 
Clarke, I., T. B. Flaherty, N. D. Wright and R. M. McMillen (2009): Student Intercultural Proficiency from Study Abroad Programs. Journal of Marketing Education, 31(2), 173-181.

Coate, S. and M. Conlin (2004): A Group Rule: Utilitarian Approach to Voter Turnout: Theory and Evidence. American Economic Review, 94(5), 1476-1504.

Conte, S., M. Conlin and A. Moro (2008): The Performance of Pivotal-Voter Models in Small-Scale Elections: Evidence from Texas Liquor Referenda. Journal of Public Economics, 92(3-4), 582-596.

Contes, D. and B. R. Humphreys (2006): Proximity Benefits and Voting on Stadium and Arena Subsidies. Journal of Urban Economics, 59(2), 285-299.

Cohn, E., S. Cohn, D. C. BAlch and J. Bradley Jr. (2004): Determinants of Undergraduate GPAs: SAT scores, High-School GPA and High-School Rank. Economics of Education Review, 23(6), 577-586.

Cowan, S. (2006): Network Regulation. Oxford Review of Economic Policy, 22(2), 248-259.

Cyrenne, P. and A. Chan (2012): High School Grades and University Performance: A Case Study. Economics of Education Review, 31(5), 524-542.

DAAD and HIS (2013): Wissenschaft Weltoffen 2013: Facts and Figures on the International Nature of Studies and Research in Germany. Bielefeld: Bertelsmann.

DALEn, D. M. (1998): Yardstick Competition and Investment Incentives. Journal of Economics \& Management Strategy, 7(1), 105-126.

Danilowicz-Gösele, K., J. Meya, R. Schwager and K. Suntheim (2014): Determinants of Students' Success at University. Cege Discussion Papers, No. 214. 
De LA O, A. L. (2013): Do Conditional Cash Transfers Affect Electoral Behavior? Evidence from a Randomized Experiment in Mexico. American Journal of Political Science, 57(1), 1-14.

DeAcon, R. and P. Shapiro (1975): Private Preference for Collective Goods Revealed through Voting on Referenda. American Economic Review, 65(5), 943-955.

DellaVigna, S. and U. Malmendier (2006): Paying not to Go to the Gym. American Economic Review, 96(3), 694-719.

Di Pietro, G. (2013): Do Study Abroad Programs Enhance the Employability of Graduates? IZA Discussion Paper, No. 7675.

DiPrete, T. A. and M. Gangl (2004): Assessing Bias in the Estimation of Causal Effects: Rosenbaum Bounds on Matching Estimators and Instrumental Variables Estimation with Imperfect Instruments. Sociological Methodology, 34(1), 271-310.

Dixit, A. and J. Londregan (1996): The Determinants of Success of Special Interests in Redistributive Politics. Journal of Politics, 58(4), 1132-1155.

Dobson, I. R. and E. Skuja (2005): Secondary Schooling: Tertiary Entry Ranks and University Performance. People and Place, 13(1), 53-62.

Downs, A. (1957): An Economic Theory of Democracy. New York: Harper and Row Publishers.

Dreher, A. and P. PoutvaAra (2011): Foreign Students and Migration to the United States. World Development, 39(8), 1294-1307.

Elinder, M., H. Jordahl and P. Poutvaara (2015): Promises, Policies and Pocketbook Voting. European Economic Review, 75, 177-194.

Epple, D. N. and R. E. Romano (1996): Public Provision of Private Goods. Journal of Political Economy, 104(1), 57-84. 
Epple, D. N., T. Romer and H. Sieg (2001): Interjurisdictional Sorting and Majority Rule: An Empirical Analysis. Econometrica, 69(6), 1437-1465.

Faure-Grimaud, A. and S. Reiche (2006): Dynamic Yardstick Mechanisms. Games and Economic Behavior, 54(2), 316-335.

Fehr, E. and K. M. Schmidt (1999): A Theory of Fairness, Competition, and Cooperation. Quarterly Journal of Economics, 114(3), 817-868.

Feld, L. P. and G. Kirchgässner (2001): The Political Economy of Direct Legislation: Direct Democracy and Local Decision-Making. Economic Policy, 16(33), 329-367.

Feld, L. P. and J. G. Matsusaka (2003): Budget Referendums and Government Spending: Evidence from Swiss Cantons. Journal of Public Economics, 87(12), 2703-2724.

FinkenstÄDt, V. and T. Kessler (2012): Die sozioökonomische Struktur der PKV-Versicherten: Ergebnisse der Einkommens- und Verbrauchsstichprobe 2008. WIP Discussion Paper, No. 3/2012.

Fiorina, M. P. (1978): Economic Retrospective Voting in American National Elections: A Micro-Analysis. American Journal of Political Science, 22(2), 426-443.

Fiorina, M. P. (1981): Retrospective Voting in American National Elections. New Haven: Yale University Press.

Fong, C. (2001): Social Preferences, Self-Interest, and the Demand for Redistribution. Journal of Public Economics, 82(2), 225-246.

Funk, P. (2010): Social Incentives and Voter Turnout: Evidence from the Swiss Mail Ballot System. Journal of the European Economic Association, 8(5), 1077-1103. 
Funk, P. and C. Gathmann (2011): Does Direct Democracy Reduce the Size of Government? New Evidence from Historical Data, 1890-2000. Economic Journal, 121(557), 1252-1280.

Gangl, M. (2004): RBOUNDS: Stata Module to Perform Rosenbaum Sensitivity Analysis for Average Treatment Effects on the Treated. https://ideas . repec.org/c/boc/bocode/s438301.html. Version 1.1.6.

Gerber, A. S., D. P. Green and C. W. Larimer (2008): Social Pressure and Voter Turnout: Evidence from a Large-Scale Field Experiment. American Political Science Review, 102(1), 33-48.

Gerdes, H. and B. Mallinckrodt (1994): Emotional, Social, and Academic Adjustment of College Students: A Longitudinal Study of Retention. Journal of Counseling and Development, 72(3), 281-288.

Girves, J. E. and V. Wemmerus (1988): Developing Models of Graduate Student Degree Progress. The Journal of Higher Education, 59(2), 163-189.

Grebennikov, L. and I. Skaines (2009): University of Western Sydney Students at Risk: Profile and Opportunities for Change. Journal of Institutional Research, 14(1), 58-70.

Harsanyi, J. C. (1980): Rule Utilitarianism, Rights, Obligations and the Theory of Rational Behavior. Theory and Decision, 12(2), 115-133.

Heckman, J. J., H. Ichimura and P. E. TodD (1998): Matching as an Econometric Evaluation Estimator. Review of Economic Studies, 65(2), 261-294.

Hillman, A. L. (2010): Expressive Behavior in Economics and Politics. European Journal of Political Economy, 26(4), 403-418.

Hillman, A. L., K. Metsuyanim and N. Potrafke (forthcoming): Democracy with Group Identity. European Journal of Political Economy.

Hong, S. (1984): The Age Factor in the Prediction of Tertiary Academic Success. Higher Education Research and Development, 3(1), 61-70. 
Key, V. O. (1966): The Responsible Electorate. Cambridge: Belknap Press of Harvard University Press.

Kinder, D. R. and D. R. Kiewiet (1979): Economic Discontent and Political Behavior: The Role of Personal Grievances and Collective Economic Judgments in Congressional Voting. American Journal of Political Science, 23(3), 495-527.

Krass, S. and M. SCHERF (2012): Warum die Einser-Inflation nicht überrascht. Süddeutsche Zeitung, November 19, 2012. http: //www . sueddeutsche.de/bildung/gute-noten-an-hochschulen-warumdie-einser-inflation-nicht-ueberrascht-1.1526490 (accessed July 14, 2014).

Krause, K.-L., R. Hartley, R. James and C. McInnes (2005): The First Year Experience in Australian Universities: Findings from a Decade of National Studies. Melbourne: University of Melbourne, Centre for the Study of Higher Education. http://www.cshe.unimelb.edu.au/research/ experience/docs/FYEReport05KLK.pdf (accessed October 20, 2013).

Kriner, D. L. and A. Reeves (2012): The Influence of Federal Spending on Presidential Elections. American Political Science Review, 106(2), 348-366.

Laffont, J.-J. (1994): The New Economics of Regulation Ten Years After. Econometrica, 62(3), 507-537.

Laffont, J.-J. and J. Tirole (1993): A Theory of Incentives in Procurement and Regulation. Cambridge: The MIT Press.

LazeAR, E. P. and S. Rosen (1981): Rank-Order Tournaments as Optimum Labor Contracts. Journal of Political Economy, 89(5), 841-864.

Leuven, E. and B. Sianesi (2003): PSMATCH2: Stata Module to Perform Full Mahalanobis and Propensity Score Matching, Common Support Graphing, and Covariate Imbalance Testing. http://ideas.repec .org/c/boc/bocode/ s432001.html. Version 4.0.4. 
LevitT, S. D. and J. M. Snyder (1997): The Impact of Federal Spending on House Election Outcomes. Journal of Political Economy, 105(1), 30-53.

Levy, S. and J. Murray (2005): Tertiary Entrance Scores Need Not Determine Academic Success: An analysis of Student Performance in an Equity and Access Program. Journal of Higher Education Policy and Management, 27(1), 129-141.

Lewis-Beck, M. S. and M. Stegmaier (2007): Economic Models of Voting. In R. J. Dalton and H.-D. Klingemann (eds.), The Oxford Handbook of Political Behavior, Oxford: Oxford University Press, 518-537.

Lindbeck, A. and J. W. Weibull (1993): A Model of Political Equilibrium in a Representative Democracy. Journal of Public Economics, 51(2), 195-209.

Littlechild, S. C. (1983): Regulation of British Telecommunications' Profitability: Report to the Secretary of State. London: Department of Industry, Her Majesty's Stationery Office.

Manacorda, M., E. Miguel and A. Vigorito (2011): Government Transfers and Political Support. American Economic Journal: Applied Economics, 3(3), $1-28$.

Margalit, Y. (2013): Explaining Social Policy Preferences: Evidence from the Great Recession. American Political Science Review, 107(1), 80-103.

Markus, G. B. (1988): The Impact of Personal and National Economic Conditions on the Presidential Vote: A Pooled Cross-Sectional Analysis. American Journal of Political Science, 32(1), 137-154.

Maskin, E. and J. Tirole (1988a): A Theory of Dynamic Oligopoly, I: Overview and Quantity Competition with Large Fixed Costs. Econometrica, 56(3), 549-569.

Maskin, E. and J. Tirole (1988b): A Theory of Dynamic Oligopoly, II: Price Competition, Kinked Demand Curves, and Edgeworth Cycles. Econometrica, $56(3), 571-599$. 
MatsusakA, J. G. (1995): Fiscal Effects of the Voter Initiative: Evidence from the Last 30 Years. Journal of Political Economy, 103(3), 587-623.

McInnis, C., R. James and R. Hartley (2000): Trends in the First Year Experience in Australian Universities. Canberra: Department of Education, Training and Youth Affairs.

McKenzie, K. and R. D. Schweitzer (2001): Who Succeeds at University? Factors Predicting Academic Performance in First Year Australian University Students. Higher Education Research and Development, 20(1), 21-33.

McNabb, R., S. Pal and P. Sloane (2002): Gender Differences in Educational Attainment: The Case of University Students in England and Wales. Economica, 69(275), 481-503.

Meltzer, A. H. and S. F. Richard (1981): A Rational Theory of the Size of Government. Journal of Political Economy, 89(5), 914-927.

Meran, G. and C. v. Hirschhausen (2009): A Modified Yardstick Competition Mechanism. Journal of Regulatory Economics, 35(3), 223-245.

Messer, D. and S. C. Wolter (2007): Are Student Exchange Programs Worth It? Higher Education, 54(5), 647-663.

Messer, K. D., G. L. Poe, D. Rondeau, W. D. Schulze and C. A. Vossler (2010): Social Preferences and Voting: An Exploration Using a Novel Preference Revealing Mechanism. Journal of Public Economics, 94(3-4), 308-317.

Meya, J. (2015): Dynamics of Yardstick Regulation: Historical Cost Data and the Ratchet Effect, Cege Discussion Papers, No. 244.

Meya, J., P. PoutvaAra and R. Schwager (2015): Pocketbook Voting and Social Preferences in Referenda. CESifo Working Paper, No. 5267.

Meya, J. and K. Suntheim (2014): The Second Dividend of Studying Abroad: The Impact of International Student Mobility on Academic Performance, Cege Discussion Papers, No. 215. 
Mizutani, F., H. Kozumi and N. Matsushima (2009): Does Yardstick Regulation Really Work? Empirical Evidence from Japan's Rail Industry. Journal of Regulatory Economics, 36(3), 308-323.

OECD (2012): How Many Students Study Abroad and Where Do They Go? Education at a Glance 2012: Highlights, OECD Publishing, http://dx.doi .org/ 10.1787/eag_highlights-2012-9-en .

OECD (2013): Economic Policy Reforms 2013: Going for Growth. Paris: OECD Publishing.

Osborne, M. J. and A. Rubinstein (1994): A Course in Game Theory. Cambridge: The MIT Press.

Parey, M. and F. Waldinger (2011): Studying Abroad and the Effect on International Labour Market Mobility: Evidence from the Introduction of Erasmus. Economic Journal, 121(551), 194-222.

Parker, J. D. A., L. J. Summerfeldt, M. J. Hogan and S. A. Majeski (2004): Emotional Intelligence and Academic Success: Examining the Transition from High School to University. Personality and Individual Differences, 36(1), 163-172.

Peat, M., J. Dalziel and A. M. Grant (2001): Enhancing the First Year Student Experience by Facilitating the Development of Peer Networks Through a OneDay Workshop. Higher Education Research and Development, 20(2), 199-215.

Pop-Eleches, C. and G. Pop-Eleches (2012): Targeted Government Spending and Political Preferences. Quarterly Journal of Political Science, 7(3), $285-320$.

Potrafke, N. (2013): Evidence on the Political Principal-Agent Problem from Voting on Public Finance for Concert Halls. Constitutional Political Economy, 24(3), 215-238. 
Potters, J., B. Rockenbach, A. SAdrieh and E. v. Damme (2004): Collusion under Yardstick Competition: An Experimental Study. International Journal of Industrial Organization, 22(7), 1017-1038.

Richter, K. (2006): Wage Arrears and Economic Voting in Russia. American Political Science Review, 100(1), 133-145.

Riker, W. H. and P. C. Ordeshook (1968): A Theory of the Calculus of Voting. American Political Science Review, 62(1), 25-42.

Robbins, S. B., K. Lauver, H. Le, D. Davis, R. Langley and A. Carlstrom (2004): Do Psychosocial and Study Skill Factors Predict College Outcomes? A Meta-Analysis. Psychological Bulletin, 130(2), 261-288.

Rogoff, K. and A. Sibert (1988): Elections and Macroeconomic Policy Cycles. The Review of Economic Studies, 55(1), 1-16.

Romer, T., H. Rosenthal and V. G. Munley (1992): Economic Incentives and Political Institutions: Spending and Voting in School Budget Referenda. Journal of Public Economics, 49(1), 1-33.

Rosenbaum, P. R. (2002): Observational Studies. New York: Springer.

Rosenbaum, P. R. and D. B. Rubin (1983): The Central Role of the Propensity Score in Observational Studies for Causal Effects. Biometrika, 70(1), 41-55.

Rousseau, J.-J. (2012 [1762]): On the Social Contract. Mineola, New York: Dover Publications.

Sappington, D. E. M. (2005): Regulating Service Quality: A Survey. Journal of Regulatory Economics, 27(2), 123-154.

Schumpeter, J. A. (1994 [1942]): Capitalism, Socialism and Democracy. London, New York: Routledge.

Shleifer, A. (1985): A Theory of Yardstick Competition. RAND Journal of Economics, 16(3), 319-327. 
Simpson, O. (2006): Predicting Student Success in Open and Distance Learning. Open Learning, 21(2), 125-138.

Smith, J. and R. NAYlor (2005): Schooling Effects on Subsequent University Performance: Evidence for the UK University Population. Economics of Education Review, 24(5), 549-562.

Sobel, J. (1999): A Reexamination of Yardstick Competition. Journal of Economics \& Management Strategy, 8(1), 33-60.

Statistisches Bundesamt (2009): Bildungsstand der Bevölkerung: Ausgabe 2009. Wiesbaden.

Stokey, N. L., R. E. Lucas with E. C. Prescott (1989): Recursive Methods in Economic Dynamics. Cambridge: Harvard University Press.

TAngerÅs, T. P. (2002): Collusion-proof Yardstick Competition. Journal of Public Economics, 83(2), 231-254.

Teichler, U. (2011): International Dimensions of Higher Education and Graduate Employment. In J. Allen and R. v. d. Velden (eds.), The Flexible Professional in the Knowledge Society: New Challenges for Higher Education, Dordrech, 199-220.

Thachil, T. (2014): Elite Parties and Poor Voters: Theory and Evidence from India. American Political Science Review, 108(2), 454-477.

Tinto, V. (1975): Dropout from Higher Education: A Theoretical Synthesis of Recent Research. Review of Educational Research, 45(1), 89-125.

Train, K. E. (1991): Optimal Regulation. The Economic Theory of Natural Monopoly. Cambridge: The MIT Press.

Trapmann, S., B. Hell, S. Weigand and H. Schuler (2007): Die Validität von Schulnoten zur Vorhersage des Studienerfolgs - eine Metaanalyse. Zeitschrift für Pädagogische Psychologie, 21(1), 11-27. 
Tyran, J.-R. and R. SAusgruber (2006): A Little Fairness May Induce a Lot of Redistribution in Democracy. European Economic Review, 50(2), 469-485.

Vlachos, J. (2004): Who Wants Political Integration? Evidence from the Swedish EU-Membership Referendum. Journal of Public Economics, 88(7-8), 1589-1604.

Zimmermann, J. and F. J. Neyer (2013): Do We Become a Different Person when Hitting the Road? Personality Development of Sojourners. Journal of Personality and Social Psychology, 105(3), 515-530.

Zucco, C. (2013): When Payouts Pay Off: Conditional Cash Transfers and Voting Behavior in Brazil 2002-10. American Journal of Political Science, 57(4), 810-822. 